\title{
HIGH-RESOLUTION H-BAND SPECTROSCOPY OF Be STARS WITH SDSS-III/APOGEE. I. NEW Be STARS, LINE IDENTIFICATIONS, AND LINE PROFILES
}

\author{
S. Drew Chojnowski ${ }^{1,2}$, David G. Whelan ${ }^{3}$, John P. Wisniewski ${ }^{4}$, Steven R. Majewski ${ }^{1}$, \\ Matthew Hall ${ }^{1}$, Matthew Shetrone ${ }^{5}$, Rachael Beaton ${ }^{1}$, Adam Burton ${ }^{1}$, Guillermo Damke ${ }^{1}$, \\ Steve Eikenberry ${ }^{6}$, Sten Hasselquist ${ }^{2}$, Jon A. Holtzman ${ }^{2}$, Szabolcs Mészáros ${ }^{7,8}$, David Nidever ${ }^{9}$, \\ Donald P. SchNeIder ${ }^{10,11}$, John Wilson ${ }^{1}$, Gail Zasowski ${ }^{12}$, DMitry BIZYAev ${ }^{2}$, Howard Brewington ${ }^{2}$, \\ J. Brinkmann ${ }^{2}$, Garrett Ebelke $^{2}$, Peter M. Frinchaboy $^{13}$, Karen Kinemuchi $^{2}$, Elena Malanushenko ${ }^{2}$, \\ Viktor Malanushenko ${ }^{2}$, Moses Marchante ${ }^{2}$, Daniel Oravetz ${ }^{2}$, Kaike Pan ${ }^{2}$, And Audrey Simmons ${ }^{2}$

\begin{abstract}
The Apache Point Observatory Galactic Evolution Experiment (APOGEE) has amassed the largest ever collection of multi-epoch, high-resolution $(R \sim 22,500), H$-band spectra for B-type emission line $(\mathrm{Be})$ stars. These stars were targeted by APOGEE as telluric standard stars and subsequently identified via visual inspection as Be stars based on $\mathrm{H}_{\text {I }}$ Brackett series emission or shell absorption in addition to otherwise smooth continua and occasionally nonhydrogen emission features. The 128/238 APOGEE Be stars for which emission had never previously been reported serve to increase the total number of known Be stars by $\sim 6 \%$. Because the $H$ band is relatively unexplored compared to other wavelength regimes, we focus here on identification of the $H$-band lines and analysis of the emission peak velocity separations $\left(\Delta v_{\mathrm{p}}\right)$ and emission peak intensity ratios $(\mathrm{V} / \mathrm{R})$ of the usually double-peaked $\mathrm{H}_{\mathrm{I}}$ and nonhydrogen emission lines. $\mathrm{H}_{\mathrm{I}} \mathrm{Br} 11$ emission is found to preferentially form in the circumstellar disks at an average distance of $\sim 2.2$ stellar radii. Increasing $\Delta v_{p}$ toward the weaker $\mathrm{Br} 12-\mathrm{Br} 20$ lines suggests these lines are formed interior to Br11. By contrast, the observed IR Fe II emission lines present evidence of having significantly larger formation radii; distinctive phase lags between IR Fe II and $\mathrm{H}_{\text {I }}$ Brackett emission lines further supports that these species arise from different radii in Be disks. Several emission lines have been identified for the first time including $\mathrm{C}_{\mathrm{I}} 16895$, a prominent feature in the spectra for almost a fifth of the sample and, as inferred from relatively large $\Delta v_{\mathrm{p}}$ compared to the Br11-Br20, a tracer of the inner regions of Be disks. Emission lines at $15760 \AA$ and $16781 \AA$ remain unidentified, but usually appear along with and always have similar line profile morphology to $\mathrm{Fe}$ II 16878 . Unlike the typical metallic lines observed for Be stars in the optical, the $H$-band metallic lines, such as Fe II 16878, never exhibit any evidence of shell absorption, even when the $\mathrm{H}_{\mathrm{I}}$ lines are clearly shell-dominated. The first known example of a quasi-triple-peaked Br11 line profile is reported for HD 253659, one of several stars exhibiting intra- and/or extraspecies V/R and radial velocity variation within individual spectra. Br11 profiles are presented for all discussed stars, as are full APOGEE spectra for a portion of the sample.
\end{abstract}

Key words: circumstellar matter - infrared: stars - line: identification - line: profiles - stars: early-type - stars: emission-line, Be

Supporting material: machine-readable and VO tables

\section{INTRODUCTION}

Since the first observational description (Struve 1931) of the characteristic double-peaked emission lines of classical Be stars, a wealth of research has demonstrated that the emission lines are formed in geometrically thin, equatorial circumstellar disks fed by gas ejected from the surfaces of rapidly rotating B stars (Porter \& Rivinius 2003; Rivinius et al. 2013b). Rapid rotation is certainly involved in the formation of these disks, but a comprehensive model of Be disk formation has yet to be created and efforts toward one are complicated by factors including the lack of examples of critically rotating Be stars, star-specific peculiarities, and the requirement of an "on/off" switch to the Be phenomenon. For an uncertain but non-negligible fraction of $\mathrm{Be}$ stars, the disks are transient, appearing in one epoch but not another (McSwain et al. 2009; Wisniewski et al. 2010). A variable rotation speed $(v \sin i)$ that occasionally reaches or exceeds the critical breakup limit is an attractive concept for such a phenomenon (Rivinius et al. 2013c) that needs to be explored 
for a sample of transient Be stars. Non-radial pulsation and turbulence due to small-scale magnetic fields remain the most likely mechanisms, along with rapid rotation, responsible for the creation of the disks (Rivinius et al. 2013b). When the disks are present, they appear to undergo Keplerian rotation (Meilland et al. 2007; Wheelwright et al. 2012) and many of the observational signatures are consistent with those predicted by the viscous decretion disk model (Lee et al. 1991; Carciofi et al. 2009; Carciofi 2011).

Multi-wavelength studies of Be disks are particularly valuable for diagnosing their structure because emission at different wavelengths originates from different physical locations within the disks (Carciofi 2011). However, unlike in the optical wavelength regime where they have been extensively studied at high spectral and temporal resolution, only a limited number of Be star surveys have been performed at near-infrared (NIR) wavelengths, and these have typically utilized low spectral resolution (Steele \& Clark 2001) and small sample sizes (Murdoch et al. 1994; Clark \& Steele 2000; Mennickent et al. 2009; Granada et al. 2010). Detailed NIR spectroscopic studies of individual Be stars are more common (e.g., Hony et al. 2000; Mathew et al. 2012) and have been used to better diagnose the gas distribution within Be disks, including the structure of onearmed density waves (Wisniewski et al. 2007; Štefl et al. 2009; Carciofi et al. 2009).

The Apache Point Observatory Galactic Evolution Experiment (APOGEE; Majewski 2012) is actively providing the first ever bulk view of the high-resolution $H$-band properties and variability of Be stars. APOGEE is one of four surveys comprising the Sloan Digital Sky Survey III (SDSS-III; Eisenstein et al. 2011). While the primary goal of the APOGEE survey is to measure the dynamical and chemical history of the Milky Way Galaxy using high-resolution $H$-band spectroscopic observations of $10^{5}$ red giant branch (RGB) stars, APOGEE devotes 35 fibers per 300-fiber pointing to observe hot stars as telluric standards. This, in addition to the surveys large sky coverage and multi-epoch observing strategy, has made APOGEE ideal for serendipitous $\mathrm{Be}$ discoveries and highresolution NIR time series data of Be stars.

Here, we present the first catalog of APOGEE Be (ABE) stars. An overview of the APOGEE survey and APOGEE data is provided in Section 2, the Be sample is described in Section 3, and the identifications of observed metallic emission lines are discussed in Section 4. Sections 5 and 6 focus on quantitative and comparative analysis of emission double-peak separation $\left(\Delta v_{\mathrm{p}}\right)$ and double-peak intensity ratios (V/R). Commentary on the more unusual or exceptional Be stars within the $\mathrm{ABE}$ sample is interspersed throughout, and an atlas of $\mathrm{Br} 11$ profiles is provided in Section 7. The Appendix includes supplemental figures displaying full APOGEE spectra for stars with strong emission features, as well as an expanded stellar data table. Future work will focus on the observed spectral variability of sources with multi-epoch APOGEE data as well as follow-up optical spectroscopy for a subset of the sample.

\section{APOGEE OVERVIEW}

\subsection{APOGEE Instrument and Observations}

The APOGEE instrument is a 300 fiber, $R \sim 22,500$ spectrograph (Wilson et al. 2010) attached to the SDSS $2.5 \mathrm{~m}$ telescope (Gunn et al. 2006) at Apache Point Observatory.
APOGEE records a vacuum wavelength range of 15145-16955 via an arrangement of three Teledyne H2RG $2048 \times 2048$ detectors. The detector layout consists of "blue," "green," and "red" detectors which cover 15145-15808 $̊, 15858-16433 \AA$, and 16474-16955 $\AA$ respectively, resulting in coverage gaps between 15808-15858 $\AA$ and 16433-16474 $\AA$. Dispersion varies with wavelength, but the central dispersions of the blue, green, and red detectors are $0.326 \AA \mathrm{pix}^{-1}, 0.283 \AA \mathrm{pix}^{-1}$, and $0.236 \AA^{\mathrm{pix}^{-1}}$ respectively. As with the original SDSS spectrograph (Smee et al. 2013), APOGEE fibers are plugged into custom, pre-drilled aluminum plates which are loaded into the telescope's focal plane and which can cover $3^{\circ}$ diameter areas of sky. Each fiber has a $2^{\prime \prime}$ field of view.

The APOGEE survey uses the Two Micron All Sky Survey (2MASS; Skrutskie et al. 2006) as a source catalog and focuses on observations of known or photometrically likely RGB stars for its main science objective (230/300 fibers per plate). For calibration purposes, blank sky (35/300 fibers) and blue telluric standards (35/300 fibers) are also observed. Typical exposure times are 1 hour, and the number of repeat observations per field is approximately equal to the number of 1 hour observations needed to reach a combined signal-to-noise ratio $(\mathrm{S} / \mathrm{N})$ per raw pixel of 100 for stars at the field-specific $H$ magnitude limit. The bright limit for science targets is always $H=7.0$, while the faint limit is variable and can be $H=11.0$ (1 hour visit), $H=12.2$ ( 31 hour visits), $H=12.8$ (6 1 hour visits), $H=13.3$ (12 1 hour visits), or $H=13.8$ (24 1 hour visits). Cohorts of RGB targets with similar $H$ magnitudes are exchanged in and out of the observing sequence as $\mathrm{S} / \mathrm{N} \sim 100$ is reached.

\subsection{APOGEE Telluric Standard Stars}

Hot O- and B-type (OB) stars are ideal candidates for telluric standard stars (TSS's) in the $H$ band because the associated spectra are relatively featureless (Meyer et al. 1998; Steele \& Clark 2001). Selection of the TSS for each APOGEE field is based on $H$ magnitude and non-reddening-corrected color rather than on intrinsic spectral properties (Zasowski et al. 2013), such that the TSS for a given field are simply the apparent bluest available stars. Thus, APOGEE makes no distinction between "normal" and emission-line stars other than to prevent from selection as TSS any stars that are reddened with respect to other stars (e.g., dusty B[e] stars) in the $3^{\circ}$ fields.

Unlike the RGB science targets, TSS are restricted to $5.5 \leqslant H \leqslant 11.0$ and are therefore always expected to reach $\mathrm{S} / N \geqslant 100$ in the typical hour exposures. In addition, the TSS for each APOGEE field are generally "locked-in," meaning that they are observed every time their respective fields are observed rather than being traded in and out of the sequence as are the RGB stars. A more comprehensive description of TSS selection is presented in Zasowski et al. (2013).

\subsection{Apache Point Observatory Galactic Evolution Experiment Spectra}

There are several details worth noting about the APOGEE spectra. Vacuum wavelengths given in angstroms $(\AA)$ are used in APOGEE data and throughout this paper. Some of the spectra were recorded during APOGEE commissioning, prior to the instrument having achieved optimal focus. The resolution of red detector data in APOGEE spectra taken prior to $\mathrm{MJD}<55804$ (September 2011) is $R \sim 16,000$, 

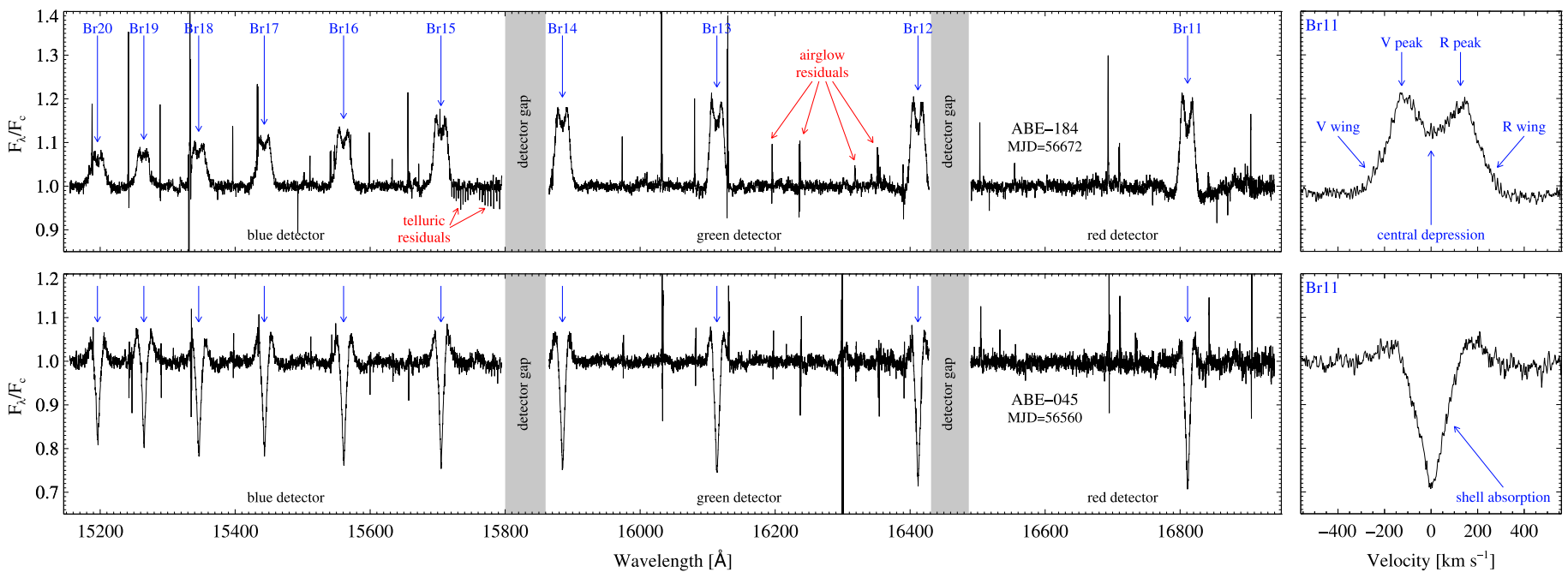

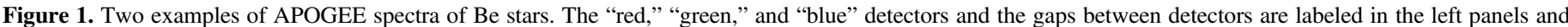

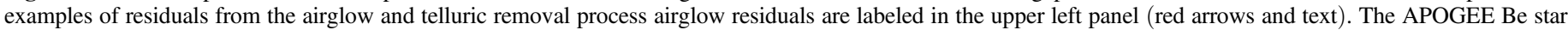

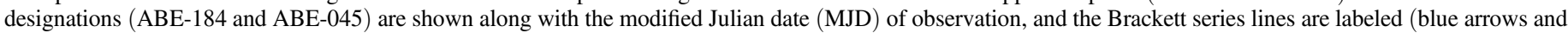

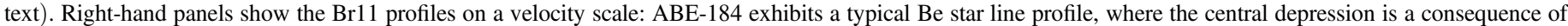

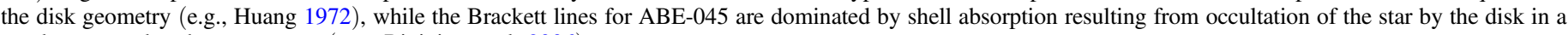
nearly or exactly edge-on system (e.g., Rivinius et al. 2006).

while the resolution is $R \sim 22,500$ for all blue and green detector data regardless of date and for all post-55804 red detector data. The raw data is processed by an automated reduction pipeline (Nidever, in preparation) that extracts the spectra, performs flat-field and wavelength calibration, and performs sky and telluric corrections. APOGEE's reduction pipeline is designed to use sky and TSS exposures (see Section 2.2) to remove airglow and telluric absorption lines from the high-resolution spectra. Because the airglow removal process has not yet been perfected, residuals from partially subtracted airglow lines remain in the final reduced data products.

Since the APOGEE survey focuses on chemical abundance and radial velocity (RV) analysis, flux standard stars are not observed and therefore the spectra are not flux-calibrated. All spectra displayed in this paper were continuum normalized using the CONTINUUM task in IRAF by fitting low-order splines to sections of blank continuum adjacent to $\mathrm{H}_{\mathrm{I}}$ Brackett lines, separately for each detector. Quoted emission line intensities and $\mathrm{V} / \mathrm{R}$ intensity ratios refer to intensity relative to normalized continuum level $\left(F_{\lambda} / F_{\mathrm{c}}\right)$. Due to the proximity of the $\operatorname{Br} 12$ and Br14 lines to the coverage limits of the green detector (21 and $27 \AA$ respectively), it was at times difficult to achieve a continuum fit that did not result in obviously incorrect intensity levels for those with respect to the other Brackett lines. The tendency of the full-width at continuum level for Brackett series emission lines to well exceed $1000 \mathrm{~km} \mathrm{~s}^{-1}$ was a further complication in salvaging the $\mathrm{Br} 12$ and $\mathrm{Br} 14$ lines, which are are de-emphasized from analysis for these reasons.

Figure 1 displays examples of APOGEE spectra for two newly-discovered $\mathrm{Be}$ stars, demonstrating the three-detector arrangement and associated coverage gaps. The ABE star IDs and modified Julian dates (MJD) of observation are provided above or below "red detector" continuum level, and commonly observed emission lines (see Section 3.5) are labeled with blue text and arrows. Examples of airglow residuals are noted with red text and arrows. The right-hand panels show Br11 line profiles from the same spectra on a velocity scale, with the line profile features of interest labeled. In most cases, Br11 is the strongest hydrogen line covered as well as the hydrogen line least likely to be affected by airglow or telluric contaminants should those be a significant issue. In subsequent figures, narrow contaminants (airglow and hot/cold/bad pixels) have been carefully trimmed from the spectra so as to avoid distraction from the features of astrophysical importance.

\subsection{Public Availability of the Spectra}

Both proprietary and publicly available spectra are used and displayed in this paper. The publicly available spectra were included in SDSS data release 10 (DR10: pertains to APOGEE data taken prior to MJD $=56112$ ), and the full data set will be made publicly available in SDSS data release 12 (DR12: scheduled for 2014 December). Shortly after DR12, we intend to convert the ABE star spectra to the format accepted by the Be Star Spectra Database (BeSS; Neiner et al. 2011) and deposit them there, ensuring convenient public access. More details on DR10-released APOGEE data can be found on the SDSS-III website (https:// www.sdss3.org/dr10/irspec/).

\section{THE ABE SAMPLE \\ 3.1. Sample Description}

The sample at hand consists of $238 \mathrm{Be}$ stars that have been observed by APOGEE a total of 1082 times. Of the 238 ABE stars, 202 were identified through periodic visual inspection of APOGEE spectra and 36 were targeted intentionally to expand the subset of previously known Be stars.

We measured the velocity separations $\left(\Delta v_{\mathrm{p}}\right)$ of the violet $(\mathrm{V})$ and red $(\mathrm{R})$ emission peaks of all lines with well-defined peaks in all spectra of sufficient quality (typically $\mathrm{S} / N>50$; dependent on emission strength) using the SPLOT feature of IRAF. Measurements pertaining to emission peaks coincident in wavelength position with strong airglow lines or diffuse interstellar bands (DIBs) (see Section 4.1) were thrown out. 
Table 1

List of ABE Stars

\begin{tabular}{|c|c|c|c|c|c|c|}
\hline $\begin{array}{l}\mathrm{ABE} \\
\mathrm{ID}\end{array}$ & $\begin{array}{c}\text { 2MASS } \\
\text { Designation }\end{array}$ & $\begin{array}{c}\text { Star } \\
\text { Name }\end{array}$ & $\begin{array}{c}\text { 2MASS } \\
H \\
\text { (mag.) }\end{array}$ & $\begin{array}{c}\text { Lit. } \\
\text { Spectral } \\
\text { Type }\end{array}$ & Ref. & $\begin{array}{c}\Delta v_{\mathrm{p}} \\
\mathrm{Br} 11 \\
\left(\mathrm{~km} \mathrm{~s}^{-1}\right)\end{array}$ \\
\hline 001 & $20212485+3722482$ & VES 203 & 9.108 & $\mathrm{~B} 0.5 \mathrm{Ve}$ & 74 & 208 \\
\hline 002 & $20151525+3654562$ & HD 228576 & 9.888 & $\mathrm{Ae}$ & 29 & 152 \\
\hline 003 & $20162816+3703229$ & HR 7757 & 6.548 & B6IIIe & 22 & 305 \\
\hline 004 & $20234596+3830033$ & HD 229221 & 6.734 & B0.2IIIe & 66 & 115 \\
\hline 005 & $20184170+3759106$ & Hen 3-1876 & 9.699 & OB & 6 & 271 \\
\hline 006 & $19124025-0627316$ & HD 179405 & 8.084 & $\mathrm{~B} 2 \mathrm{Ve}$ & 68 & 294 \\
\hline 007 & $19104149-0542581$ & HD 178920 & 9.236 & $\mathrm{~B} 8 \mathrm{II} / \mathrm{III}$ & 58 & 181 \\
\hline 008 & $18000176-2323071$ & & 10.699 & & & 233 \\
\hline 009 & $20461437+5039005$ & TYC 3586-282-1 & 9.192 & (B8) & $\mathrm{C}$ & 175 \\
\hline 010 & $20450869+5033004$ & BD+50 3188 & 9.311 & (B) & $\mathrm{C}$ & 138 \\
\hline 011 & $20535693+5005293$ & TYC 3583-670-1 & 9.698 & & & 252 \\
\hline 012 & $20554731+5040274$ & & 10.766 & & & 115 \\
\hline 013 & $18574179-0419113$ & EM* $^{*}$ CDS 1038 & 10.428 & $\mathrm{OB}$ & 24 & bl \\
\hline 014 & $21300088+4529390$ & HD 204860 & 6.931 & $\mathrm{~B} 5.5 \mathrm{Ve}$ & 60 & 326 \\
\hline 015 & $18123846-2708292$ & HD 166629 & 9.155 & B5nne & 41 & 70 \\
\hline 016 & $18185069-1227145$ & HD 168135 & 7.406 & $\mathrm{~B} 8 \mathrm{Ve}$ & 59 & 238 \\
\hline 017 & $18122758-1546123$ & BD-15 4863 & 9.695 & $\mathrm{Be}$ & 24 & 376 \\
\hline 018 & $18194798-1724130$ & & 10.976 & & & $\mathrm{sp}$ \\
\hline 019 & $23533653+5649116$ & HD 223924 & 8.177 & B1.5:III:n & 25 & 296 \\
\hline 020 & $18432516-0339100$ & SS 412 & 10.528 & OB:e & 33 & 217 \\
\hline 021 & $04220085+5430434$ & HD 232940 & 8.625 & (B9) & $\mathrm{C}$ & 291 \\
\hline 022 & $04254177+5615294$ & TYC 3727-1849-1 & 9.438 & & & 495 \\
\hline 023 & $03282223+4507560$ & $\mathrm{BD}+44$ 709s & 10.546 & $\mathrm{OB}$ & 14 & 386 \\
\hline 024 & $05113282+2408029$ & TYC 1846-17-1 & 9.596 & (A3) & $\mathrm{C}$ & 158 \\
\hline 025 & $05452088+2909281$ & HD 247042 & 9.165 & O9.5: & 15 & 346 \\
\hline 026 & $05485364+2908100$ & HD 38708 & 8.015 & B3:e:psh & 8 & 324 \\
\hline 027 & $05453713+3007253$ & TYC 2405-1358-1 & 9.825 & & & 489 \\
\hline 028 & $06123985+3258216$ & HD 42529 & 8.090 & B9V & 57 & 297 \\
\hline 029 & $06165595+3414299$ & HD 254168 & 9.169 & & & 301 \\
\hline 030 & $06185921+3413502$ & HD 43681 & 8.806 & (A2) & 39 & 333 \\
\hline 031 & $06273614+1815476$ & HD 257473 & 9.378 & $\mathrm{~B} 5 \mathrm{e}$ & 5 & 133 \\
\hline 032 & $22252246+5642384$ & SS 453 & 10.200 & $\mathrm{Be}:$ & 33 & 508 \\
\hline $\mathbf{0 3 3}$ & $23181131+5550356$ & HD 240249 & 9.250 & (B8) & $\mathrm{C}$ & 205 \\
\hline 034 & $23521212+6710073$ & MWC 1085 & 8.787 & $(\mathrm{~B} 3 \mathrm{Ve})$ & 54 & 551 \\
\hline 035 & 06334350-3202486 & Hen 3-14 & 9.650 & $\mathrm{~B}$ & 24 & 241 \\
\hline 036 & $06283925-3222165$ & HR 2364 & 6.113 & $\mathrm{~B} 3 \mathrm{Ve}$ & 69 & 456 \\
\hline 037 & $05590290+3101488$ & HD 40254 & 9.210 & (B8) & 39 & 234 \\
\hline 038 & $20032620+2242411$ & HD 345589 & 10.595 & (A3) & 52 & 224 \\
\hline 039 & $18000839-2356576$ & SS 338 & 9.647 & B8e: & 33 & 259 \\
\hline 040 & $17375482-2457569$ & HD 159845 & 6.773 & B3IIIe & 45 & 99 \\
\hline 041 & $18382765-1014211$ & & 10.640 & & & 141 \\
\hline 042 & $18464650-1021523$ & BD-10 4799 & 10.094 & (B) & $\mathrm{C}$ & 181 \\
\hline 043 & $17441414-2727284$ & HD 161004 & 7.880 & B9IVe & 41 & 290 \\
\hline 044 & $17432344-2715411$ & HD 316179 & 7.673 & Be: & 35 & 247 \\
\hline 045 & $01542524+5651061$ & TYC 3692-1234-1 & 10.321 & & & 344 \\
\hline 046 & $02135183+5354525$ & HD 13544 & 9.055 & B0.5IIIn & 27 & 294 \\
\hline 047 & $05390426+3758359$ & HD 37266 & 7.309 & B8V & 57 & 250 \\
\hline 048 & $21375002+4258309$ & $\mathrm{BD}+424162$ & 8.916 & (A0) & 39 & 333 \\
\hline 049 & $21103095+4741321$ & HR 8107 & 6.360 & B6IV & 22 & 197 \\
\hline 050 & $19584848+2305215$ & HD 345439 & 10.629 & B2Vpe & 77 & 1153 \\
\hline 051 & $19571220+2158541$ & HD 345439 & 9.869 & (A0) & 52 & 373 \\
\hline 052 & $18052711-2921573$ & TYC 6854-2016-1 & 10.009 & (B8) & 61 & 405 \\
\hline 053 & $18023404-3027418$ & HD 317026 & 9.467 & (B9) & 52 & 216 \\
\hline 054 & $05064093+2230389$ & HD 32811 & 6.523 & $\mathrm{~B} 8.5 \mathrm{~V}$ & 57 & 315 \\
\hline 055 & $06591343+0427179$ & HD 51893 & 9.077 & B9V & 58 & 344 \\
\hline 056 & $00302445+6010093$ & HIP 2382 & 9.710 & B6III & 48 & as (sh) \\
\hline 057 & $02534457+6443068$ & TYC 4056-415-1 & 9.293 & (B5) & 61 & sh \\
\hline 058 & $18072725-2506165$ & HD 165517 & 6.967 & B0Iae & 45 & $\mathrm{tc}$ \\
\hline 059 & $18144388-2137597$ & HD 167113 & 9.030 & (B9IV:) & 61 & 415 \\
\hline 060 & $07134058+3806302$ & HD 55200 & 8.297 & $(\mathrm{~A} 0)$ & 39 & $\mathrm{sh}$ \\
\hline 061 & $04361726+5230217$ & HD 28942 & 8.159 & Ash & 44 & $\mathrm{sh}$ \\
\hline
\end{tabular}


Table 1

(Continued)

\begin{tabular}{|c|c|c|c|c|c|c|}
\hline $\begin{array}{l}\mathrm{ABE} \\
\mathrm{ID}\end{array}$ & $\begin{array}{c}\text { 2MASS } \\
\text { Designation }\end{array}$ & $\begin{array}{l}\text { Star } \\
\text { Name }\end{array}$ & $\begin{array}{c}\text { 2MASS } \\
H \\
\text { (mag.) }\end{array}$ & $\begin{array}{c}\text { Lit. } \\
\text { Spectral } \\
\text { Type }\end{array}$ & Ref. & $\begin{array}{c}\Delta v_{\mathrm{p}} \\
\mathrm{Br} 11 \\
\left(\mathrm{~km} \mathrm{~s}^{-1}\right)\end{array}$ \\
\hline 062 & $03030934+6654223$ & TYC 4060-96-1 & 8.398 & & & 456 \\
\hline 063 & $06351041+0634180$ & HD 260153 & 9.417 & B8III & 42 & $\mathrm{w}$ \\
\hline 064 & $18451050-0545120$ & TYC 5126-2325-1 & 10.733 & & & 291 \\
\hline 065 & $18434859-0608188$ & HD 173075 & 9.358 & B9IV & 58 & 235 \\
\hline 066 & $18402120-0455127$ & TYC 5121-940-1 & 10.299 & & & 187 \\
\hline 067 & $03292627+4656162$ & HR 1047 & 5.902 & B7Vne & 51 & 133 \\
\hline 068 & $17224642-2533273$ & HD 157174 & 8.488 & A0IV & 45 & 208 \\
\hline 069 & $18274975-1104312$ & & 10.165 & & & 238 \\
\hline 070 & 18251991-0918163 & BD-09 4724 & 9.550 & (A0IV) & $\mathrm{C}$ & w \\
\hline 071 & $18424368-1000273$ & TYC 5696-503-1 & 10.454 & $\mathrm{Be}$ & 33 & 138 \\
\hline 072 & $18043735+0155085$ & HD 165174 & 6.224 & B0IIIn & 22 & $\mathrm{w}$ \\
\hline 073 & $22583631+5528116$ & BD+54 2887 & 9.538 & $(\mathrm{~A} 0)$ & 61 & 193 \\
\hline 074 & $19233702+3859363$ & HD 182550 & 8.818 & B8V & 47 & 66 \\
\hline 075 & $03464087+3217247$ & HD 23478 & 6.486 & B3IVe & 10 & 913 \\
\hline 076 & $04432066+4754385$ & VES 828 & 10.896 & & & 301 \\
\hline 077 & $04423114+3830469$ & & 10.455 & & & 162 \\
\hline 078 & $21351726+5647589$ & TYC 3975-1585-1 & 10.101 & B8 & 12 & 400 \\
\hline 079 & 07081479-2325541 & HD 54551 & 8.775 & B1.5II & 45 & 524 \\
\hline 080 & $20282074+4526025$ & $\mathrm{BD}+443475$ & 9.451 & & & sh \\
\hline 081 & $00104514+5801058$ & HD 628 & 7.521 & (B9) & 39 & 205 \\
\hline 082 & $05540108+1241033$ & BD+12938 & 10.172 & & & 250 \\
\hline 083 & $05453721+1311210$ & HD 247299 & 9.987 & (A0) & 62 & 119 \\
\hline 084 & $01042742+5756263$ & HD 236611 & 8.978 & (A) & $\mathrm{C}$ & 265 \\
\hline 085 & $01183306+5822304$ & HD 236689 & 8.853 & B1.5V:epsh & 8 & 321 \\
\hline 086 & $01355734+5809128$ & TYC 3683-1262-1 & 9.840 & & & 209 \\
\hline 087 & 07331124-1136421 & HD 60260 & 9.232 & $\mathrm{~B} 3 \mathrm{Ve}$ & 58 & 129 \\
\hline 088 & $01055296+6558158$ & HD 6343 & 6.843 & B5Vn:e & 20 & 115 \\
\hline 089 & $00573323+6709339$ & TYC 4029-428-1 & 9.595 & & & 342 \\
\hline 090 & $00501808+6710377$ & BD+66 64 & 8.588 & (B9) & $\mathrm{C}$ & $\mathrm{sh}$ \\
\hline 091 & $05564423+1601018$ & HD 39984 & 9.140 & (A2) & 39 & 115 \\
\hline 092 & $03250006+5029394$ & TYC 3320-1906-1 & 9.425 & $\mathrm{~B} 7$ & 9 & 417 \\
\hline 093 & $00080292+7332356$ & TYC 4306-1125-1 & 9.115 & B8V & 37 & 337 \\
\hline 094 & $00331160+5140069$ & HD 232214 & 8.855 & (B8) & $\mathrm{C}$ & 92 \\
\hline 095 & $06272285+0824429$ & HD 45396 & 8.907 & (A2) & 39 & $\mathrm{w}$ \\
\hline 096 & $06300018+0817453$ & HD 45828 & 8.506 & (B8?) & $\mathrm{C}$ & $\mathrm{w}$ \\
\hline 097 & $05164674+3022455$ & HD 34193 & 8.498 & $(\mathrm{~A} 0 \mathrm{e})$ & 39 & 203 \\
\hline 098 & $23152849+6416002$ & HD 219523 & 7.218 & B5V & 36 & 301 \\
\hline 099 & $06073989+2751354$ & HD 41639 & 8.599 & B6Vne: & 38 & 578 \\
\hline 100 & $05341240+4516410$ & HD 36467 & 8.167 & B9III & 37 & 300 \\
\hline 101 & $05124298+4754272$ & BD+47 1108 & 9.581 & $(\mathrm{~A} 0)$ & 39 & 326 \\
\hline 102 & $05003547+3552170$ & TYC 2400-1784-1 & 10.396 & & & 273 \\
\hline 103 & $17494627-2249517$ & & 10.473 & & & $<61$ \\
\hline 104 & $18411366-0247380$ & BD-02 4698 & 8.853 & (B) & $\mathrm{C}$ & $\operatorname{sh} ?$ \\
\hline 105 & $20451060+5112379$ & HD 235350 & 8.652 & B0.5IV & 8 & 502 \\
\hline 106 & $02250591+5515032$ & TYC 3690-1236-1 & 10.581 & & & 480 \\
\hline 107 & $21563126+5041249$ & TYC 3617-2074-1 & 10.112 & & & 233 \\
\hline 108 & $06185755+2323286$ & HD 254842 & 8.631 & & & 185 \\
\hline 109 & $06231994+2506057$ & HD 256137 & 9.734 & (A2) & 39 & 106 \\
\hline 110 & $04365908+5217135$ & HD 29035 & 7.930 & $\mathrm{~B} 9.5 \mathrm{Ve}$ & 64 & $\mathrm{w}$ \\
\hline 111 & $18581515-0528567$ & AS 332 & 9.639 & $\mathrm{Be}$ & 43 & w \\
\hline 112 & $04321707+4816572$ & HD 28543 & 7.725 & (A0) & 39 & $\mathrm{w}$ \\
\hline 113 & $04363913+4104368$ & HD 29096 & 7.323 & B8IV & 57 & 333 \\
\hline 114 & $04460607+4705516$ & TYC 3347-1615-1 & 10.713 & & & sh? \\
\hline 115 & $20185676+3745319$ & & 11.326 & & & 139 \\
\hline 116 & $17331509-1922379$ & HD 159032 & 8.725 & B9IV & 45 & 387 \\
\hline 117 & $17515926-3029411$ & HD 162345 & 8.291 & (B8) & 39 & 128 \\
\hline 118 & $17534729-2945087$ & HD 316573 & 9.850 & (B9) & 52 & 115 \\
\hline 119 & $17531191-2857284$ & AS 251 & 9.961 & $\mathrm{~B}$ & 24 & 259 \\
\hline 120 & $17521395-2744257$ & HD 316475 & 9.225 & (B9) & 39 & 282 \\
\hline 121 & $17525570-2218434$ & TYC 6262-3203-1 & 9.292 & & & 166 \\
\hline 122 & $18194176-1058093$ & TYC 5681-507-1 & 10.027 & (A5) & 62 & 179 \\
\hline
\end{tabular}


Table 1

(Continued)

\begin{tabular}{|c|c|c|c|c|c|c|}
\hline $\begin{array}{l}\mathrm{ABE} \\
\mathrm{ID}\end{array}$ & $\begin{array}{c}\text { 2MASS } \\
\text { Designation }\end{array}$ & $\begin{array}{l}\text { Star } \\
\text { Name }\end{array}$ & $\begin{array}{c}\text { 2MASS } \\
H \\
\text { (mag.) }\end{array}$ & $\begin{array}{c}\text { Lit. } \\
\text { Spectral } \\
\text { Type }\end{array}$ & Ref. & $\begin{array}{c}\Delta v_{\mathrm{p}} \\
\mathrm{Br} 11 \\
\left(\mathrm{~km} \mathrm{~s}^{-1}\right)\end{array}$ \\
\hline 123 & $18220126-1048042$ & TYC 5681-151-1 & 10.614 & & & 177 \\
\hline 124 & $18161427-2906365$ & HD 167401 & 9.322 & B4II/III & 41 & 627 \\
\hline 125 & $18221389-1307360$ & TYC 5689-54-1 & 10.273 & & & $<60$ \\
\hline 126 & $18245968-1406408$ & HD 169418 & 8.995 & B9.5III & 45 & 326 \\
\hline 127 & $18205460-1243598$ & HD 168566 & 8.710 & B9III & 45 & 114 \\
\hline 128 & $18404465-0758241$ & HIP 91591 & 8.825 & $\mathrm{~B} 8 \mathrm{Ve}$ & 49 & 276 \\
\hline 129 & $18385819-0827466$ & GSC 05692-00540 & 10.451 & B7 & 17 & 267 \\
\hline 130 & $18405017-0741018$ & GSC 05692-00399 & 10.508 & B7 & 17 & 423 \\
\hline 131 & $18395898-0733138$ & BD-07 4647 & 9.642 & B5 & 17 & 143 \\
\hline 132 & $18355878-0744307$ & BD-07 4630 & 8.963 & B9 & 17 & w \\
\hline 133 & $17500331+4823391$ & 88 Her & 6.913 & B6IIInpsh & 53 & 285 \\
\hline 134 & $18295996-0908375$ & & 10.761 & & & 198 \\
\hline 135 & $18432970-0919127$ & HD 173010 & 7.179 & O9.7Ia & 73 & $\operatorname{sh} ?$ \\
\hline 136 & $18064578-2821496$ & HD 165365 & 7.024 & B7.5III & 41 & 232 \\
\hline 137 & $18042703-2228572$ & NGC 6531 F195 & 11.130 & & & $<25$ \\
\hline 138 & $19150144+0948272$ & HD 180126 & 7.565 & B2IV & 68 & 365 \\
\hline 139 & $19164642+1058468$ & HD 180587 & 7.578 & Apsh & 18 & $\mathrm{sh}$ \\
\hline 140 & $20234436+3728351$ & HR 7807 & 6.228 & B2Vne & 63 & 382 \\
\hline 141 & $21061887+2824477$ & HD 201036 & 8.996 & $\mathrm{~B} 6 / 8 \mathrm{Vn}$ & 38 & 247 \\
\hline 142 & $19270008+1632172$ & HD 183035 & 7.844 & A0V & 70 & 320 \\
\hline 143 & $20040584+3009117$ & HD 333378 & 10.150 & (A0) & 52 & 313 \\
\hline 144 & $20005874+3113497$ & HD 189847 & 7.122 & B7V & 16 & 260 \\
\hline 145 & $17530194-2219531$ & TYC 6262-1413-1 & 9.926 & & & sh? \\
\hline 146 & $06072002+2640558$ & HD 41600 & 7.132 & B9IV & 57 & $\mathrm{w}$ \\
\hline 147 & $19424993+4239003$ & HD 186485 & 8.484 & B9V & 34 & 123 \\
\hline 148 & $20002133+2135515$ & HD 345506 & 9.683 & (B8) & 52 & sh? \\
\hline 149 & $03434449+3143092$ & IRASF03406+3133 & 10.780 & & & $\mathrm{sp}$ \\
\hline 150 & $18412551-0534033$ & & 10.902 & & & 388 \\
\hline 151 & $18404500-0740458$ & TYC 5692-1370-1 & 10.799 & B7 & 17 & 137 \\
\hline 152 & 07290132-0832539 & SS 120 & 10.733 & B8e: & 35 & 461 \\
\hline 153 & $17221970-2833450$ & & 10.940 & & & $\mathrm{sh}$ \\
\hline 154 & $05394249+2215279$ & TYC 1310-2084-1 & 9.969 & (B8) & 61 & 360 \\
\hline 155 & $01590196+5725521$ & TYC 3692-1671-1 & 10.611 & & & 819 \\
\hline 156 & $23380341+5556420$ & HD 222185 & 8.343 & (A2) & 39 & w \\
\hline 157 & $19450599+1617091$ & HD 186637 & 7.937 & (B9e) & 39 & 359 \\
\hline 158 & $21520306+5853123$ & AS 478 & 9.792 & & & 87 \\
\hline 159 & $22275192+6300090$ & MWC 1062 & 8.804 & B5:e & 3 & 265 \\
\hline 160 & $21365704+6811073$ & HD 206135 & 7.811 & B3V & 21 & 316 \\
\hline 161 & $21551055+5326166$ & TYC 3968-1354-1 & 10.574 & OB- & 14 & 422 \\
\hline 162 & $06063872+2754038$ & $\mathrm{BD}+27981$ & 9.960 & (B8) & 62 & 219 \\
\hline 163 & $22465987+5345241$ & HD 215837 & 8.104 & (A0) & 39 & 357 \\
\hline 164 & $22202269+5151395$ & HD 212044 & 7.702 & B1:V:nnep & 8 & 191 \\
\hline 165 & $22245295+5207583$ & HD 212666 & 8.681 & B5.5e & 59 & 397 \\
\hline 166 & 06460565-0558109 & TYC 4812-2496-1 & 9.971 & & & 308 \\
\hline 167 & 06495552-0530472 & HD 49787 & 7.836 & $\mathrm{~B} 1 \mathrm{Ve}$ & 68 & 300 \\
\hline 168 & 06570947-0832309 & HD 51477 & 8.223 & $\mathrm{~B} 3 \mathrm{Ve}$ & 58 & 628 \\
\hline 169 & $06024105+2202482$ & HD 40897 & 8.000 & (B9) & 39 & 255 \\
\hline 170 & $06014161+2224036$ & HR 2116 & 6.400 & $\mathrm{~B} 8 \mathrm{~V}$ & 26 & 153 \\
\hline 171 & $06081219+2156586$ & TYC 1326-1188-1 & 10.257 & (A2) & 62 & 362 \\
\hline 172 & $05212545+1601440$ & HD 34906 & 8.641 & (B9V) & 61 & 142 \\
\hline 173 & $05175643+1519211$ & TYC 1283-1360-1 & 10.617 & & & $\mathrm{sh}$ \\
\hline 174 & $05240938+1633455$ & HD 35269 & 7.552 & $\mathrm{~A} 0 \mathrm{~V}$ & 76 & 448 \\
\hline 175 & $05214456+1709194$ & TYC 1300-652-1 & 10.731 & & & w \\
\hline 176 & $05133229+3806348$ & HD 33656 & 9.215 & B5 & 2 & 551 \\
\hline 177 & $05194374+3820304$ & HD 280849 & 9.654 & (B3) & 52 & 522 \\
\hline 178 & $06450343-0034140$ & CoRoT 102762536 & 11.651 & B1V & 75 & $\mathrm{sp}$ \\
\hline 179 & $06450928-0115205$ & EM* RJHA 51 & 10.563 & B5Ib & 75 & 243 \\
\hline 180 & $06422978+0053582$ & $\mathrm{EM}^{*}$ RJHA 40 & 10.613 & $\mathrm{~B} 3 \mathrm{Ib}$ & 75 & 183 \\
\hline 181 & $05355408-0537423$ & HD 37115 & 7.204 & $\mathrm{~B} 7.5 \mathrm{~V}$ & 58 & 238 \\
\hline 182 & $06123495+4145095$ & TYC 2934-118-1 & 10.240 & & & 376 \\
\hline 183 & 07364229-1303499 & HD 60993 & 9.131 & B2II & 45 & 348 \\
\hline
\end{tabular}


Table 1

(Continued)

\begin{tabular}{|c|c|c|c|c|c|c|}
\hline $\begin{array}{l}\mathrm{ABE} \\
\mathrm{ID}\end{array}$ & $\begin{array}{c}\text { 2MASS } \\
\text { Designation }\end{array}$ & $\begin{array}{l}\text { Star } \\
\text { Name }\end{array}$ & $\begin{array}{c}\text { 2MASS } \\
H \\
\text { (mag.) }\end{array}$ & $\begin{array}{c}\text { Lit. } \\
\text { Spectral } \\
\text { Type }\end{array}$ & Ref. & $\begin{array}{c}\Delta v_{\mathrm{p}} \\
\mathrm{Br} 11 \\
\left(\mathrm{~km} \mathrm{~s}^{-1}\right)\end{array}$ \\
\hline 184 & $05361555+3257145$ & HD 245174 & 9.790 & (B3) & 62 & 268 \\
\hline 185 & $05580029+2437017$ & HD 40132 & 7.564 & B9 & 19 & 85 \\
\hline 186 & $07075533+0143105$ & HD 54167 & 9.672 & B5/7Ib: & 58 & 328 \\
\hline 187 & $06121659+2005097$ & HD 253214 & 8.917 & B1.5:V:nn & 8 & 220 \\
\hline 188 & $06121386+2000034$ & HD 253215 & 10.444 & & & 298 \\
\hline 189 & $07370572+1654153$ & HD 60848 & 7.071 & O9.5IVe & 67 & 305 \\
\hline 190 & $06110671+1810591$ & HD 252904 & 9.089 & B9V & 11 & w \\
\hline 191 & $18084894-1858344$ & HD 165854 & 7.903 & B9e & 59 & 274 \\
\hline 192 & $18103823-1910006$ & HD 166291 & 8.303 & B3II & 45 & 272 \\
\hline 193 & $18011841-2721498$ & SS 339 & 10.713 & B8e: & 33 & 250 \\
\hline 194 & $18173492-1842282$ & HD 313062 & 9.676 & & & 327 \\
\hline 195 & $19120326+0237212$ & HD 179343 & 6.613 & B8III & 68 & 266 \\
\hline 196 & $05010612+4134002$ & HD 277241 & 10.836 & B8 & 13 & $\mathrm{w}$ \\
\hline 197 & $18095327-2302251$ & HD 166055 & 9.662 & (B9) & 62 & 221 \\
\hline 198 & $18091443-2246378$ & HD 165894 & 8.183 & $\mathrm{~B} 3 \mathrm{IV} / \mathrm{V}$ & 45 & 158 \\
\hline 199 & $17554711-2142367$ & TYC $6262-371-1$ & 9.301 & & & 164 \\
\hline 200 & $18235550-1547477$ & Lan 671 & 9.976 & & & 280 \\
\hline 201 & $18282453-1642195$ & BD-16 4888p & 9.825 & & & 138 \\
\hline 202 & $18283909-1512088$ & TYC 6266-143-1 & 10.487 & & & w \\
\hline $\mathrm{A} 01$ & $00201742+6227498$ & MWC 5 & 8.025 & B0.5IV & 7 & 122 \\
\hline $\mathrm{A} 02$ & $00320285+6709401$ & HD 2789 & 7.519 & B3:Vne & 23 & 320 \\
\hline A03 & $04042164+5319447$ & MWC 80 & 7.165 & B1Vnnpe & 8 & as \\
\hline A04 & $05251782+2936535$ & HD 35347 & 7.948 & B2:nne & 38 & 268 \\
\hline A05 & $05445623+2127384$ & HD 38191 & 8.380 & B1:V:ne: & 8 & 246 \\
\hline A06 & $05503228+1801349$ & HD 39018 & 7.898 & (B9) & 39 & w \\
\hline A07 & $06183944+2300285$ & HD 43703 & 7.475 & B1IV:p? & 8 & $\mathrm{w}$ \\
\hline A08 & $06330559+1656553$ & HD 46264 & 7.522 & B5Vne & 31 & 409 \\
\hline A09 & $06333223+0820080$ & HD 259597 & 7.779 & B1Vnne & 28 & 289 \\
\hline A10 & $06525305-1000270$ & HD 50424 & 8.971 & B8e & 35 & 178 \\
\hline A11 & $06545882-0342013$ & HD 50891 & 7.880 & $\mathrm{~B} 0.5 \mathrm{Ve}$ & 68 & 215 \\
\hline A12 & $06561908-0348254$ & HD 51193 & 8.039 & B1.5IVe & 68 & 358 \\
\hline A13 & $06574289+1754071$ & HD 51354 & 7.183 & $\mathrm{~B} 3 \mathrm{Vn}$ & 40 & 257 \\
\hline A14 & 07093697-1605467 & HD 54786 & 9.051 & B1.5Ib: & 45 & 256 \\
\hline A15 & 07133410-0204390 & HD 55606 & 8.704 & B0.5Vnnep & 69 & 237 \\
\hline A16 & $19525141+2214226$ & HD 345122 & 8.963 & $\mathrm{~B} 3 \mathrm{Ve}$ & 46 & 233 \\
\hline A17 & $20024644+2151160$ & HD 190150 & 8.186 & B6IV-Ve & 40 & 241 \\
\hline A18 & $21082962+4715254$ & HD 201522 & 8.022 & B0V & 16 & \\
\hline A19 & $21250244+4427063$ & MWC 640 & 7.206 & B1.5V:nnep & 8 & 205 \\
\hline $\mathrm{A} 20$ & $21291483+4420173$ & HD 204722 & 7.643 & B1.5IV:np & 25 & 358 \\
\hline $\mathrm{A} 21$ & $22013820+5010046$ & MWC 649 & 8.701 & $\mathrm{~B} 3 \mathrm{e}^{\mathrm{P}}$ & 1 & 109 \\
\hline A22 & $22060834+4954088$ & AS 483 & 9.631 & B1.5V:nne: & 8 & 362 \\
\hline $\mathrm{A} 23$ & $18211606-1301256$ & MWC 922 & 7.396 & unclB $[\mathrm{e}]$ & 55 & $\mathrm{sp}$ \\
\hline A24 & $05181018+3739003$ & HD 34302 & 7.534 & (B8) & 39 & 163 \\
\hline A25 & $05231490+3742536$ & HD 280999 & 9.582 & (B3) & 52 & 214 \\
\hline A 26 & $05254477+3538499$ & HD 35345 & 7.851 & B1Vep & 7 & $\mathrm{sp}$ \\
\hline A 27 & $05280968+3516540$ & $\mathrm{EM}^{*} \mathrm{CDS} 496$ & 8.669 & $\mathrm{OB}$ & 24 & w? \\
\hline A 28 & $05595354+2505190$ & HD 250028 & 8.083 & B2:V:nep & 32 & 288 \\
\hline A 29 & $05530609+2626435$ & HD 39340 & 7.579 & $\mathrm{~B} 3 \mathrm{Ve}$ & 59 & 197 \\
\hline $\mathrm{A} 30$ & $05533110+2544321$ & HD 248753 & 7.361 & B2:Vnne & 23 & 279 \\
\hline A31 & $05535984+2625212$ & HD 39478 & 7.691 & $\mathrm{~B} 2 \mathrm{Ve}$ & 59 & 242 \\
\hline A32 & $06072661+2205477$ & IGR J06074+2205 & 10.189 & $\mathrm{~B} 0.5 \mathrm{Ve}$ & 71 & 380 \\
\hline $\mathrm{A} 33$ & $06074953+1839264$ & EM* LkHA 208 & 9.834 & $\mathrm{~A} 7 \mathrm{e}$ & 65 & $\mathrm{sp} ?$ \\
\hline A34 & $06065436+1902040$ & HD 251726 & 7.644 & B1V:e & 8 & 159 \\
\hline A 35 & $06184553+1516522$ & MWC 137 & 7.840 & $\operatorname{sgB}[e]$ & 56 & 57 \\
\hline A36 & $06135416+1631049$ & HD 253659 & 8.327 & B0.5:V:nne & 8 & 312 \\
\hline Q01 & $20240654+3829332$ & HD 229239 & 7.093 & B0.2III & 66 & \\
\hline Q02 & $20194162+3811060$ & HD 228932 & 9.386 & B & 35 & \\
\hline Q03 & $20213867+3725151$ & $\mathrm{BD}+364032$ & 7.566 & $08.5 \mathrm{~V}$ & 66 & \\
\hline Q04 & $20213589+3721395$ & Hen 3-1885 & 10.855 & $\mathrm{~A} 0 \mathrm{~V}$ & 30 & \\
\hline Q05 & $06382991+0042351$ & HD 291946 & 9.321 & B9 & 2 & \\
\hline
\end{tabular}


Table 1

(Continued)

\begin{tabular}{|c|c|c|c|c|c|c|}
\hline $\begin{array}{l}\mathrm{ABE} \\
\mathrm{ID}\end{array}$ & $\begin{array}{c}\text { 2MASS } \\
\text { Designation }\end{array}$ & $\begin{array}{l}\text { Star } \\
\text { Name }\end{array}$ & $\begin{array}{c}\text { 2MASS } \\
H \\
\text { (mag.) }\end{array}$ & $\begin{array}{c}\text { Lit. } \\
\text { Spectral } \\
\text { Type }\end{array}$ & Ref. & $\begin{array}{c}\Delta v_{\mathrm{p}} \\
\mathrm{Br} 11 \\
\left(\mathrm{~km} \mathrm{~s}^{-1}\right)\end{array}$ \\
\hline Q06 & 18071193-2516305 & HD 315177 & 10.022 & & & \\
\hline Q07 & $19582996+2033006$ & HD 350989 & 10.534 & B7IIIn & 50 & \\
\hline Q08 & $20012170+2217258$ & HD 345475 & 9.484 & B0 & 4 & \\
\hline Q09 & $23204452+6111404$ & EM $^{*}$ CDS 1459 & 7.471 & O6.5(f)(n)p & 72 & \\
\hline Q10 & $00350607+6258585$ & EM* $^{*}$ CDS 53 & 10.369 & OB-e: & 14 & \\
\hline Q11 & $00310135+5539101$ & HD 232208 & 9.519 & B3:e & 3 & \\
\hline Q12 & $00255124+7148258$ & HD 2083 & 7.041 & O9.5III & 34 & \\
\hline Q13 & $01243585+5812454$ & $\mathrm{EM}^{*}$ CDS 144 & 10.499 & OB-e: & 14 & \\
\hline Q14 & $04310304+4146289$ & HD 276414 & 10.148 & (B8) & 52 & \\
\hline Q15 & $04390489+4115001$ & HD 29332 & 8.246 & B3ne & 1 & \\
\hline Q16 & $05085056+4144262$ & HD 32961 & 8.970 & B2 & 13 & \\
\hline Q17 & $04360336+3640031$ & HD 280006 & 7.747 & A0Ibe: & 33 & \\
\hline Q18 & $06321639+0110289$ & HD 288805 & 9.611 & B5 & 2 & \\
\hline Q19 & 06594264-1109265 & HD 52159 & 9.793 & B3Vne & 69 & \\
\hline Q20 & $05204307+3726192$ & HD 34656 & 6.634 & O7.5(f)II & 73 & \\
\hline Q21 & 07213463-0553498 & HD 57539 & 6.834 & B3IV & 58 & \\
\hline Q22 & $06154017+0603582$ & HR 2231 & 6.336 & B6Ve & 69 & \\
\hline Q23 & $22061730+6355026$ & 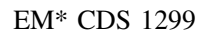 & 10.236 & OB & 24 & \\
\hline
\end{tabular}

References. (1) Merrill et al. (1942); (2) Cannon \& Mayall (1949); (3) Merrill \& Burwell (1949); (4) Popper (1950); (5) Miller \& Merrill (1951); (6) Nassau \& Harris (1952); (7) Morgan et al. (1953); (8) Morgan et al. (1955); (9) Heckmann et al. (1956); (10) Hiltner (1956); (11) Duflot et al. (1958); (12) Alknis (1958); (13) McCuskey (1959); (14) Hardorp et al. (1959); (15) Bouigue et al. (1961); (16) Fehrenbach et al. (1962); (17) Roslund (1963); (18) Feast \& Thackeray (1963); (19) McCuskey (1967); (20) Schmidt-Kaler (1967); (21) Racine (1968); (22) Lesh (1968); (23) Guetter (1968); (24) Wackerling (1970); (25) Walborn (1971); (26) Cowley (1972); (27) Lesh \& Aizenman (1973); (28) Turner (1976); (29) Henize (1976); (30) Voroshilov et al. (1976); (31) Davis (1977); (32) Christy (1977); (33) Stephenson \& Sanduleak (1977); (34) Hill \& Lynas-Gray (1977);(35) Stephenson \& Sanduleak (1977); (36) Roman (1978); (37) Bartaya (1979); (38) Clausen \& Jensen (1979); (39) Ochsenbein (1980); (40) Jaschek \& Jaschek (1993); (41) Houk (1982); (42) Voroshilov et al. (1985); (43) Bopp (1988); (44) Bidelman (1988); (45) Houk \& Smith-Moore (1988); (46) Radoslavova (1989); (47) Sato \& Kuji (1990); (48) Turner et al. (1992); (49) Grillo et al. (1992); (50) Turner (1993); (51) Garrison \& Gray (1994); (52) Nesterov et al. (1995); (53) Abt \& Morrell (1995); (54) Kohoutek \& Wehmeyer (1997); (55) Lamers et al. (1998); (56) Esteban \& Fernandez (1998); (57) Grenier et al. (1999); (58) Houk \& Swift (1999); (59) Yudin (2001); (60) Chauville et al. (2001); (61) Kharchenko (2001); (62) Fabricius et al. (2002); (63) Abt et al. (2002); (64) Miroshnichenko et al. (2003); (65) Hernández et al. (2004); (66) Negueruela (2004); (67) Negueruela et al. (2004);(68) Frémat et al. (2006); (69) Levenhagen \& Leister (2006); (70) Uzpen et al. (2007); (71) Reig et al. (2010); (72) Walborn et al. (2010); (73) Sota et al. (2011); (74) Mathew \& Subramaniam (2011); (75) Sebastian et al. (2012); (76) Chargeishvili et al. (2013); (77) Eikenberry et al. (2014).

No attempt was made to remove underlying photospheric absorption prior to $\Delta v_{p}$ measurement.

Table 1 provides the ABE identifiers, star names, 2MASS $H$ magnitudes, literature spectral types and references where available (see Section 3.2), and the mean $\Delta v_{\mathrm{p}}$ for the Br11 line from all APOGEE spectra for each source. Star names beginning with "J" are 2MASS designations, and newly identified Be stars are indicated by bold font for the ABE ID. If a $\Delta v_{p}$ measurement for the Br11 line could not be made in any of the available spectra despite evidence of Br11 emission or shell absorption, one of the following abbreviations is provided in place of a $\Delta v_{p}$ value: "w" weak emission-peaks not discernible; "sp" single-peaked emission; "sh" shell absorption without resolved adjacent emission peaks; "as" severe asymmetry in emission peak heights such that only one peak is discernible (not the same as single-peaked); "bl" V peak of Br11 is severely blended with Fe II 16792 (ABE-013); "tc" spectra are heavily contaminated by telluric features (ABE-058).

The ABE identifiers were assigned to avoid the use of sometimes lengthy survey identifiers which are the only star names available. Three groups of ABE stars are distinguished from one another by ABE ID as follows:

(1) ABE-001-ABE-202 refer to Be stars that were quasirandomly targeted by APOGEE as TSS and subsequently identified as Be stars through visual inspection of the wavelength region encompassing Br11 and Fe II 16878. To account for sources only producing emission lines in certain epochs, which was frequently the case, it was necessary to examine all $>70,000$ individual spectra for all $>17,000$ telluric stars.

(2) ABE-A01-ABE-A36 refer to Be stars that we targeted intentionally via internal proposals for APOGEE observations of ancillary (hence the "A" prefix of the ABE IDs) science targets falling within a subset of pre-planned APOGEE fields. Most of the intentionally targeted Be stars are early-type (B3 and hotter) classical Be stars, showing stronger than average $H$-band emission in the APOGEE spectra, but two stars classified as $\mathrm{B}[\mathrm{e}]$ in the literature were observed (ABE-A23 and ABE-A35) as was a reported Herbig Ae star (ABE-A33).

(3) ABE-Q01-ABE-Q23 refer to stars which (a) had existing "emission line star" classifications in the literature, (b) appeared to be hot OBA stars in the APOGEE spectra, but (c) did not produce any discernible emission in any of the associated APOGEE data (all have multi-epoch data), or in others words, were $H$-band quiescent (hence the "Q" of the ABE IDs) during the observations.

\subsection{Literature Spectral Types}

The He I 17007 line, analogous to optical He I in terms of utility as an effective temperature $\left(T_{\text {eff }}\right)$ diagnostic for OB stars 
(Blum et al. 1997; Meyer et al. 1998) and the only nonhydrogen stellar absorption feature expected to be present for B-type stars (the earliest-O stars exhibit He II 15723, 16923 absorption), is not covered by APOGEE spectra. Therefore, detailed spectral classification of OB stars is not possible with these data and the literature was perused for the existing spectral classifications included in Table 1.

The Catalogue of Stellar Spectral Classification (CSSC; Skiff 2013) was the primary resource used for locating spectral type information, but some of the original sources of spectral types in the CSSC (primarily those pre-dating 1940) could not be tracked down. In those cases, the spectral types are enclosed in parentheses and the provided reference is "C." Other secondhand spectral types, culled from modern compilations of historical data, are also enclosed in parentheses. The spectral types not enclosed in parentheses are therefore those that could be linked directly to the paper or catalog where the spectral type was determined or estimated.

\subsection{New Be Star Discoveries}

A total of $128 \mathrm{Be}$ stars have been identified as Be stars for the first time via Brackett series emission in APOGEE spectra. According to the BeSS (Neiner et al. 2011), which maintains a comprehensive database of classical $\mathrm{Be}$ and main sequence $\mathrm{B}[\mathrm{e}]$ stars, $2070 \mathrm{Be}$ stars are cataloged in the Milky Way and Magellanic clouds combined. The 128 new Be stars presented in this work therefore represent a $\sim 6.2 \%$ increase in the number of known Be stars.

The positions of all 238 ABE stars are shown in Figure 2, along with the Be star entries included in the BeSS database (Neiner et al. 2011). Although APOGEE observes a large number of fields in the Galactic Halo, the majority (90\%) of Be stars observed during the survey reside along the plane of the Milky Way (at Galactic latitudes, $|b|<10$ ), similar to the trend seen in BeSS.

Stars included in the ABE sample were generally required to exhibit evidence of emission or shell absorption in at least the $\mathrm{H}_{\mathrm{I}} \mathrm{Br} 11$ line. The exceptions to this rule are ABE-111, ABE196, and ABE-A06; these stars appear clearly to have emission from Fe II 16878 (see Section 4.2) despite very weak or no emission in the Brackett lines. Figure 3 shows examples of new and previously known Be stars representing the most borderline cases included the ABE sample. For many of these stars, the Br11 emission is sufficiently weak that double-peaks are not discernible. Rather, the photospheric Br11 absorption wings appear filled in with emission, creating "shoulders" on the line profiles (e.g., ABE-112) and making them easily distinguishable from purely photospheric lines profiles. Fe II emission is also apparent for a number of these stars, despite weakness of the $\mathrm{H}$ I emission.

The large number of new Be stars identified by APOGEE is due in large part to the high-resolution, high-S/N spectra which permit identification of very weak disk signatures (e.g., Figure 3) that might be overlooked in lower-resolution spectra or narrow-band photometry. Repeated observations of most of the stars $(>1$ observation for $93 \%$ of sample) can provide confirmation of very weak disk signatures and also reveals transient Be disks, where Brackett series emission either fades away or appears unexpectedly from epoch to epoch (J. P. Wisniewski 2015, in preparation). Among the reasons for 86/ 128 newly identified ABE stars having been classified in the literature as normal O-, B-, or A-type stars is that the stars did not possess CS disks at the time the spectral types were determined or estimated.

\subsection{ABE-144 and ABE-170: the Brightest New Be Stars}

The brightest newly identified Be star among the $\mathrm{ABE}$ sample is ABE-170, a.k.a. HR $2116(V=6.36)$, and the second brightest is ABE-144, a.k.a. HD $189847(V=6.92)$. The lack of a prior indicator of emission lines for ABE-144 may be due to few or no historical spectroscopic observations of the star beyond Fehrenbach et al. (1962). It is unclear whether or not Balmer series emission would have been noticed in that study. ABE-170 has been observed spectroscopically in more recent studies including Abt et al. (2002) and Strom et al. (2005), but the spectra used in both of those papers were limited in wavelength coverage to the region encompassing $\mathrm{He}_{\mathrm{I}} 4471$ and $\mathrm{Mg}$ II 4481, such that emission at $\mathrm{H} \alpha$ or $\mathrm{H} \beta$ would not have been recognized if present. Among the possible reasons for the $\mathrm{Be}$ nature of these stars not having been previously recognized is that ABE-144 and ABE-170 were normal B stars during past observations (similar to, e.g., Bjorkman \& Miroshnichenko 2000).

\subsection{Observed Emission Lines}

The emission lines detected in APOGEE's wavelength range are listed in Table 2. For each line, Table 2 provides the (1) line identification, (2) laboratory rest wavelength, (3) observed wavelength (see concluding paragraph of this section), (4) the difference between laboratory and observed wavelengths, (5) lower level energy $E_{i},(6)$ upper level energy $E_{k}$, (7) transition strength expressed as $\log \left(g_{i} f_{i k}\right),(8)$ for metallic lines only, the number of confident and possible detections, (9) the number of stars for which $\Delta v_{p}$ was measured, (10) the range of $\Delta v_{p}$ measurements, (11) the average of all $\Delta v_{\mathrm{p}}$ measurements, (12) and other transitions possibly contributing to the observed emission line profiles.

Attempts to identify all non-hydrogen (metallic) lines, described in Section 4, made use primarily of Peter van Hoof's Atomic Line List v2.05b16 ${ }^{14}$ (PLL from here on) and to a lesser extent the NIST Atomic Database (Kramida et al. 2013) and the Kurucz line list. ${ }^{15}$ The identities of metallic emission lines at 15760 and $16781 \AA$ remain ambiguous due to few transitions around the correct wavelengths having available transition probability data needed for confident identification. These lines, referred to as $\lambda 15760$ and $\lambda 16781$ throughout this paper, are discussed in more detail in Section 4.5. Since forbidden line emission was present for only one source, ABE-A23 a.k.a. MWC 922, the central star of the Red Square Nebula (Tuthill \& Lloyd 2007), Table 2 is limited to the permitted (E1) transitions observed for Be stars. The $H$-band spectrum of MWC 922 is sufficiently different from the rest of the sample and sufficiently more complex that an in-depth analysis is currently being pursued separately (Whelan, in preparation).

The observed wavelengths as well as the differences between laboratory and observed wavelengths, provided in columns (4) and (5) of Table 2, pertain to the average position of double emission peaks for each line plus a correction factor based on

\footnotetext{
14 http://www.pa.uky.edu/ peter/newpage/

151995 Atomic Line Data (R.L. Kurucz and B. Bell) Kurucz CD-ROM No. 23. Cambridge, MA: Smithsonian Astrophysical Observatory.
} 


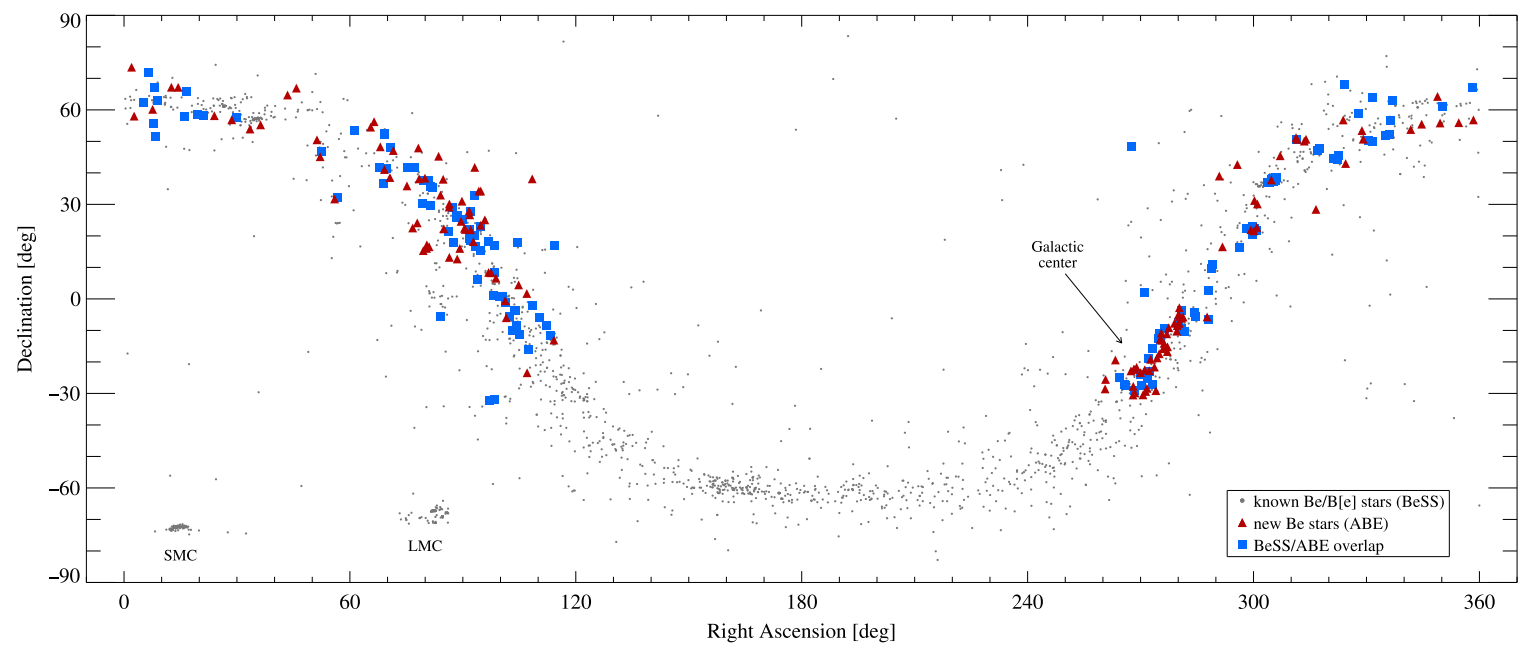

Figure 2. This adaptation of Neiner et al. (2011) Figure 1 shows the RA and decl. positions of all the BeSS entries as black dots, known Be stars observed by APOGEE as squares (blue), and new Be stars discovered in the APOGEE survey as triangles (red). The Galactic Center and Magellanic Clouds are labeled.

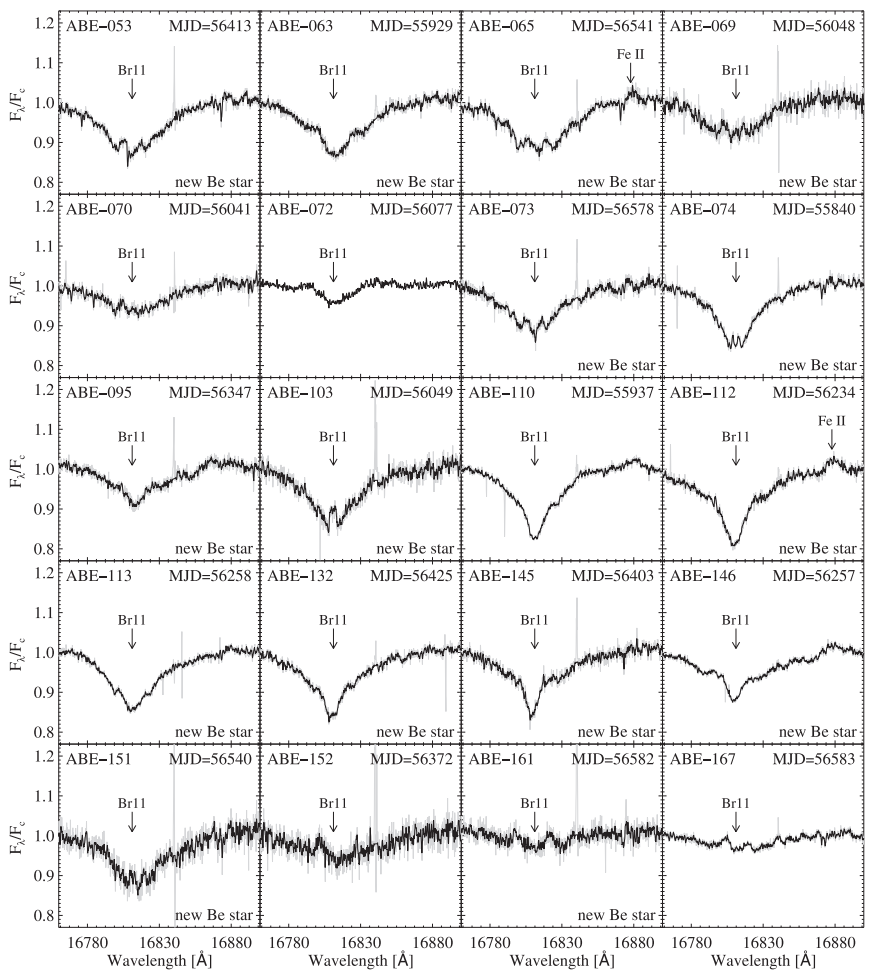

Figure 3. Br11 region for some new and previously known Be stars showing very weak evidence of circumstellar emission.

the Doppler shift found for the Br11 line. Br11 is the strongest line covered for these stars and provides the most reliable peak position measurements, so correction to rest frame was done simply by adding to the observed wavelength of each line the difference between $\mathrm{Br} 11$ emission peak midpoint and Br11 rest wavelength.

\section{NON-HYDROGEN LINE IDENTIFICATION}

\subsection{Diffuse Interstellar Bands}

The DIB at $15271 \AA$, discovered by Geballe et al. (2011), is present in most of the $\mathrm{ABE}$ spectra and in numerous
APOGEE spectra (Zasowski et al. 2014). Because DIB 15271 usually falls on or near the $\mathrm{Br} 19 \mathrm{R}$ emission peak, Br19 peak separation measurements are omitted from this paper. Examples of DIB 15271 absorption (marked with red dotted lines) in spectra for four active Be stars and two currently emission-less stars are displayed in Figure 4. Other DIBs $(15615,15651,15671 \AA$ ) discussed in Geballe et al. (2011) are present for most objects with DIB 15271, as are other possible DIBs at $\sim 15314$ and $\sim 16154 \AA$ A Of the spectra shown in Figure 4, DIB 15314 appears most prominently in the spectrum for ABE-137.

\subsection{Fe II}

The Fe II 16878 line appears in emission for between $32 \%-46 \%$ (upper limit includes weak or ambiguous detections) of the 238 active Be stars discussed here, making it the most frequently observed metallic feature in APOGEE's coverage of the $H$ band. For stars with very strong Fe II 16878, the much weaker Fe II 16792 also appears in emission but is usually blended with $\mathrm{Br} 11$. As for $\mathrm{Fe}$ II 16878, proximity of the feature to $\mathrm{C}_{\mathrm{I}} 16895$ often leads to a blend of the two lines, especially since $\mathrm{C}_{\mathrm{r}}$ emission is always broad compared to Fe II (see Section 5.2).

Examples of stars with emission from one or both $H$-band $\mathrm{Fe}$ II lines are presented in Figure 5. The left panel demonstrates the wide range of $\mathrm{H}$ I strength corresponding to $\mathrm{Fe}$ II detections. As is seen quite clearly for the lowermost stars (ABE-A06, ABE-111) in the left panel of Figure 5, Fe II emission may be present even when there is no perceptible emission from Brackett series lines, contrary to the finding of Steele \& Clark (2001). ABE-A06 has been a Be-shell star at various epochs (see BeSS spectra), but in the APOGEE data exhibits only very weak filling of the Br11 photospheric absorption wings in addition to the weak Fe II 16878 emission that, for ABE-A06, persists in four spectra sparsely covering 0.77 years. Less evidence is available for $\mathrm{HI}_{\mathrm{I}}$ emission in the case of ABE-111, despite the Fe II feature appearing in all six APOGEE covering 2.29 years. Though not shown in Figure 5, ABE-196 also lacks convincing evidence of $\mathrm{HI}_{\mathrm{I}}$ emission and yet exhibits $\mathrm{Fe}$ II 16878 emission in all 13 APOGEE spectra covering 3.02 years. Line profile variability in the Brackett lines is observed for all 
Table 2

Observed Emission Lines and Summary of $\Delta v_{\mathrm{p}}$ Measurements

\begin{tabular}{|c|c|c|c|c|c|c|c|c|c|c|c|}
\hline (1) & (2) & (3) & (4) & $(5)$ & (6) & (7) & (8) & (9) & (10) & (11) & (12) \\
\hline $\begin{array}{l}\text { Atom or } \\
\text { Ion }\end{array}$ & $\begin{array}{c}\lambda_{\mathrm{v} a c} \\
\mathrm{lab} \\
(\AA) \\
\end{array}$ & $\begin{array}{l}\lambda_{\mathrm{v} a c} \\
\mathrm{obs}^{\mathrm{a}} \\
(\AA) \\
(\AA)\end{array}$ & $\begin{array}{r}\text { Diff. lab-obs } \\
(\AA)\end{array}$ & $\begin{array}{r}E_{i} \\
(\mathrm{eV}) \\
\end{array}$ & $\begin{array}{r}E_{k} \\
(\mathrm{eV}) \\
\end{array}$ & $\log (g f)$ & $\begin{array}{c}\mathrm{N} \\
\text { detections } \\
\text { yes (maybe) }\end{array}$ & $\begin{array}{c}\Delta v_{\mathrm{p}} \\
\mathrm{N} \\
\text { stars }\end{array}$ & $\begin{array}{c}\Delta v_{\mathrm{p}} \\
\text { range } \\
\left(\mathrm{km} \mathrm{s}^{-1}\right)\end{array}$ & $\begin{array}{c}\Delta v_{\mathrm{p}} \\
\text { mean } \\
\left(\mathrm{km} \mathrm{s}^{-1}\right)\end{array}$ & $\begin{array}{c}\text { Other } \\
\text { possible } \\
\text { contribution }\end{array}$ \\
\hline H I $(\mathrm{Br} 20)$ & 15195.996 & 15195.932 & 0.064 & 12.749 & 13.564 & -1.487 & $\cdots$ & 48 & $68-566$ & 314 & $\ldots$ \\
\hline H I (Br19) & 15264.708 & $\ldots$ & $\ldots$ & 12.749 & 13.561 & -1.414 & $\ldots$ & $\ldots$ & $\ldots$ & $\ldots$ & DIB 15271 \\
\hline $\mathrm{Fe} \mathrm{I}$ & 15298.740 & 15298.528 & 0.212 & 5.309 & 6.119 & 0.650 & 6 & 3 & $188-283$ & 228 & $\ldots$ \\
\hline H I (Br18) & 15345.982 & 15345.987 & -0.005 & 12.749 & 13.556 & -1.337 & $\cdots$ & 57 & $67-533$ & 276 & $\cdots$ \\
\hline H I (Br17) & 15443.139 & 15443.187 & -0.048 & 12.749 & 13.551 & -1.255 & $\ldots$ & 73 & $66-537$ & 266 & $\ldots$ \\
\hline H I (Br16) & 15560.699 & 15560.697 & 0.002 & 12.749 & 13.545 & -1.166 & $\cdots$ & 47 & $65-436$ & 266 & $\cdots$ \\
\hline N I & 15586.545 & 15586.591 & -0.046 & 12.126 & 12.922 & -0.023 & 1 & 1 & $52-52$ & 52 & $\ldots$ \\
\hline H I (Br15) & 15704.952 & 15705.015 & -0.063 & 12.749 & 13.538 & -1.071 & $\cdots$ & 76 & $65-552$ & 282 & $\ldots$ \\
\hline $\mathrm{Mg} \mathrm{I}$ & 15753.291 & blend & $\ldots$ & 5.932 & 6.719 & 0.140 & 7 & $\cdots$ & $\ldots$ & $\ldots$ & Mg I 15745 \\
\hline$\lambda 15760$ & $\ldots$ & 15760.161 & $\ldots$ & $\ldots$ & $\ldots$ & $\ldots$ & $36(13)$ & 11 & 27-304 & 161 & $\mathrm{Mg}_{\mathrm{I}}$ \\
\hline $\mathrm{Mg} \mathrm{I}$ & 15770.149 & 15770.943 & -0.794 & 5.933 & 6.719 & 0.411 & $9(1)$ & 2 & $338-375$ & 356 & $\lambda 15760$ \\
\hline H I (Br14) & 15884.880 & 15884.875 & 0.005 & 12.749 & 13.529 & -0.967 & $\ldots$ & 91 & $61-522$ & 259 & $\ldots$ \\
\hline Si I & 15892.771 & blend & $\ldots$ & 5.082 & 5.862 & -0.007 & 6 & $\ldots$ & $\ldots$ & $\ldots$ & $\ldots$ \\
\hline Si I & 15964.422 & 15963.229 & 1.193 & 5.984 & 6.761 & 0.198 & $7(1)$ & 3 & $321-383$ & 345 & $\cdots$ \\
\hline C I & 16009.270 & blend & $\ldots$ & 9.631 & 10.406 & 0.234 & $7(3)$ & $\ldots$ & $\ldots$ & $\ldots$ & $\ldots$ \\
\hline C I & 16026.080 & blend & $\ldots$ & 9.631 & 10.405 & 0.222 & $5(5)$ & $\cdots$ & $\ldots$ & $\cdots$ & $\cdots$ \\
\hline $\mathrm{H}_{\mathrm{I}}(\mathrm{Br} 13)$ & 16113.714 & 16113.766 & -0.052 & 12.749 & 13.518 & -0.852 & $\ldots$ & 96 & $61-517$ & 249 & $\ldots$ \\
\hline H I (Br12) & 16411.674 & 16411.763 & -0.089 & 12.749 & 13.504 & -0.725 & $\cdots$ & 95 & $59-524$ & 252 & $\ldots$ \\
\hline $\mathrm{Ca}$ II & 16565.590 & 16565.973 & -0.383 & 9.235 & 9.983 & 0.368 & 6 & 3 & $208-333$ & 291 & C I 16564 \\
\hline Ca II & 16654.430 & blend & $\ldots$ & 9.240 & 9.984 & 0.626 & $1(2)$ & $\ldots$ & $\ldots$ & $\ldots$ & $\ldots$ \\
\hline Si I & 16685.341 & blend & $\ldots$ & 5.984 & 6.727 & -0.117 & $1(2)$ & $\ldots$ & $\ldots$ & $\ldots$ & $\ldots$ \\
\hline Mg II & 16764.800 & 16764.922 & -0.122 & 12.083 & 12.822 & 0.481 & $2(2)$ & 1 & $34-34$ & 34 & $\ldots$ \\
\hline$\lambda 16781$ & $\ldots$ & 16781.115 & $\ldots$ & $\ldots$ & $\ldots$ & $\ldots$ & $36(13)$ & 13 & 28-309 & 157 & $\cdots$ \\
\hline $\mathrm{Fe}$ II & 16791.762 & 16791.953 & -0.191 & 5.484 & 6.222 & -2.325 & $8(6)$ & 1 & $48-48$ & 48 & $\ldots$ \\
\hline $\mathrm{Mg} \mathrm{II}$ & 16804.520 & blend & $\ldots$ & 12.085 & 12.822 & 0.737 & $2(2)$ & $\cdots$ & $\ldots$ & $\cdots$ & Mg II 16804 \\
\hline $\mathrm{H}$ I $(\mathrm{Br} 11)$ & 16811.111 & $\ldots$ & $\ldots$ & 12.749 & 13.486 & -0.582 & $\ldots$ & 194 & $57-1153$ & 282 & $\ldots$ \\
\hline $\mathrm{Fe}$ II & 16877.808 & 16877.822 & -0.014 & 5.484 & 6.219 & -1.256 & $76(33)$ & 26 & $24-232$ & 82 & $\cdots$ \\
\hline C I & 16895.031 & 16894.898 & 0.133 & 9.003 & 9.736 & 0.534 & $43(19)$ & 14 & $31-539$ & 243 & $\ldots$ \\
\hline Si II & 16911.430 & 16911.646 & -0.216 & 12.147 & 12.880 & 0.350 & $1(1)$ & 1 & $41-41$ & 41 & $\cdots$ \\
\hline
\end{tabular}

${ }^{a}$ The emission peak midpoint corrected by the emission peak midpoint of $\operatorname{Br} 11$.

three stars and is likely due to varying degrees of emission filling, but lack of knowledge of the true photospheric absorption profiles prevents us from confidently claiming $\mathrm{H}_{\mathrm{I}}$ emission is present.

The right panel of Figure 5 focuses on some of the more extreme Be stars in this sample, starting with the obvious outlier ABE-A23, an unclassified B[e] star (Lamers et al. 1998) for which the exceptionally strong $\mathrm{Fe}_{\mathrm{II}}$ and [Fe II] emission lines reported by Rudy et al. (1992) dominate the APOGEE spectra. In contrast to ABE-A23, where the emission lines all appear truly single-peaked, ABE-137 is likely a classical Be star viewed at an inclination, $i$, of nearly or exactly zero. The Brackett series lines for ABE-137 show some peak structure even though the peaks are not resolved. The Fe II 16878 line is very narrow and pointed but double peaks are resolved in the C I 16895 line, suggesting the presence of a circumstellar disk. ABE-A35 exhibits strong Fe II emission, and is the only source of the ABE sample for which the $\Delta v_{\mathrm{p}}$ of Fe II 16792 could be measured. The average Fe in peak separations from five spectra for $\mathrm{ABE}-\mathrm{A} 35$ are in good agreement: $\Delta v_{\mathrm{p}}(\mathrm{Fe}$ II 16878) $=$ $52.6 \pm 2.53 \mathrm{~km} \mathrm{~s}^{-1}, \Delta v_{\mathrm{p}}\left(\right.$ Fe II 16792) $=48.3 \pm 4.01 \mathrm{~km} \mathrm{~s}^{-1}$. The lower resolution of red detector data from APOGEE commissioning data is likely a factor in the single-peaked appearance of the Fe II lines for ABE-015.

\subsection{Fe II Profiles as a Function of Inclination}

In past studies, optical Fe II lines have been used (Hanuschik 1996) to establish a strict definition of the shell, or edge-on, class of Be stars. Photospheric Fe II absorption lines are usually observed at greatest strength for A-F supergiants (Gray \& Corbally 2009), so if the central depression of an Fe II emission line for a Be star extends below undisturbed, adjacent continuum level, the implication is that the the disk is viewed at sufficiently large inclination that our line of sight passes through an appreciable volume of cool gas in the inner, equatorial disk. It is a well-known fact that Fe II and Ti II shell lines are among the strongest metallic features present in the spectra of edge-on Be stars.

In contrast to the observed behavior of optical Fe II lines, stars with obvious shell absorption in the Brackett series lines exhibit no evidence of shell absorption in the $H$-band Fe II lines nor in any of the covered metallic lines, such that the Fe II line profile shapes for pole-on Be stars differ from those of edge-on Be stars only in line width. This fact is demonstrated in Figure 6, where the upper panel compares Brl1 and Fe II profiles for five stars viewed over a range of inclination angles. As can be seen, the $\mathrm{Fe}$ II profiles are pure emission regardless of the what form the $\mathrm{H}_{\mathrm{I}}$ profiles take. The lower right panel of Figure 6 presents additional examples of $\mathrm{H}$ I-shell stars with 


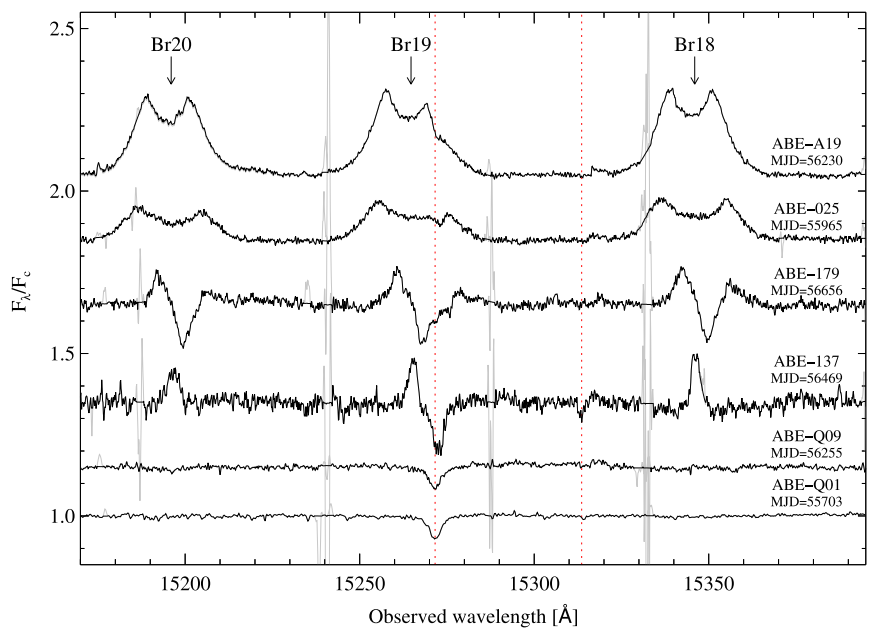

Figure 4. Spectra for six stars with visible DIB 15271 absorption around or on the Br19 line. No correction for radial velocity has been applied to the spectra. The dotted lines (red) mark DIB 15271 and another likely DIB at $~ 15314$ that appears in numerous APOGEE spectra.

Fe II emission, while the lower left panel (as well as the edgeon example in the upper panel) highlights ABE-035, the most extreme shell star within this sample in terms of Brackett series shell depth.

\section{4. $C_{I}$ and Other Neutral Lines}

An emission line at $16895 \AA$ is identified for the first time as Ci 16895.031 and is observed for between $18-26 \%$ of the 238 Be stars. Figure 5 displays 11 examples of stars with $\mathrm{C}_{\mathrm{I}}$ emission and the strongest detections will be discussed in Section 4.4.1. A C 16895 absorption line is present in numerous APOGEE spectra of A-F stars, but the line is typically not present for OB stars unless in emission. Except in the case of very narrow-lined Be stars (e.g., ABE-015, ABE040 in Figure 5), the R peak of the $\mathrm{C}_{\mathrm{I}} 16895$ emission profile is frequently compromised by a strong airglow line around $\sim 16904$ A.

Prior mentions in the literature of NIR C I emission include Groh et al. (2007) and Štefl et al. (2009), where several $C_{I}$ emission lines were detected around $10700 \AA$ in $\mathrm{Be}$ star spectra. Spectra showing C I 16895 emission have been included in a number of papers, but the line is usually either confused and/or blended with $\mathrm{Fe}$ II 16878, or not identified at all. Ashok \& Banerjee (2000) noticed the C I 16895 line in a subset of low-resolution Be star spectra and realized that it was probably not $\mathrm{Fe}$ II 16878 due to the measured wavelength of the line $(\sim 16893 \AA$, or $\sim 15 \AA$ from the $\mathrm{Fe}$ II wavelength). Kendall et al. (2003) presented medium resolution $H$-band spectra of three young stellar object (YSO) candidates, one of which, IRAS 17441-2910, was found to be a very strong emission line source. A plot of the spectrum shows singlepeaked $\mathrm{Br} 11$ and $\mathrm{Fe}$ II 16878 emission and strong doublepeaked C I 16895 emission, but the authors did not comment on the latter.

NIR emission from $\mathrm{C}_{\mathrm{I}}$ is not limited to classical Be stars. $\mathrm{C}_{\mathrm{I}}$ 16895 emission was present in a high-resolution spectrum shown by Kraus et al. (2012) for the Herbig B[e] star V921 Scorpii, and the $\mathrm{C}_{\mathrm{I}}$ emission line is also present in APOGEE spectra for both $\mathrm{B}[\mathrm{e}]$ stars observed by APOGEE to date (see right-hand panel of Figure 5). Even luminous blue variable stars display evidence of $\mathrm{C}_{\mathrm{I}}$ emission lines (Groh et al. 2007),
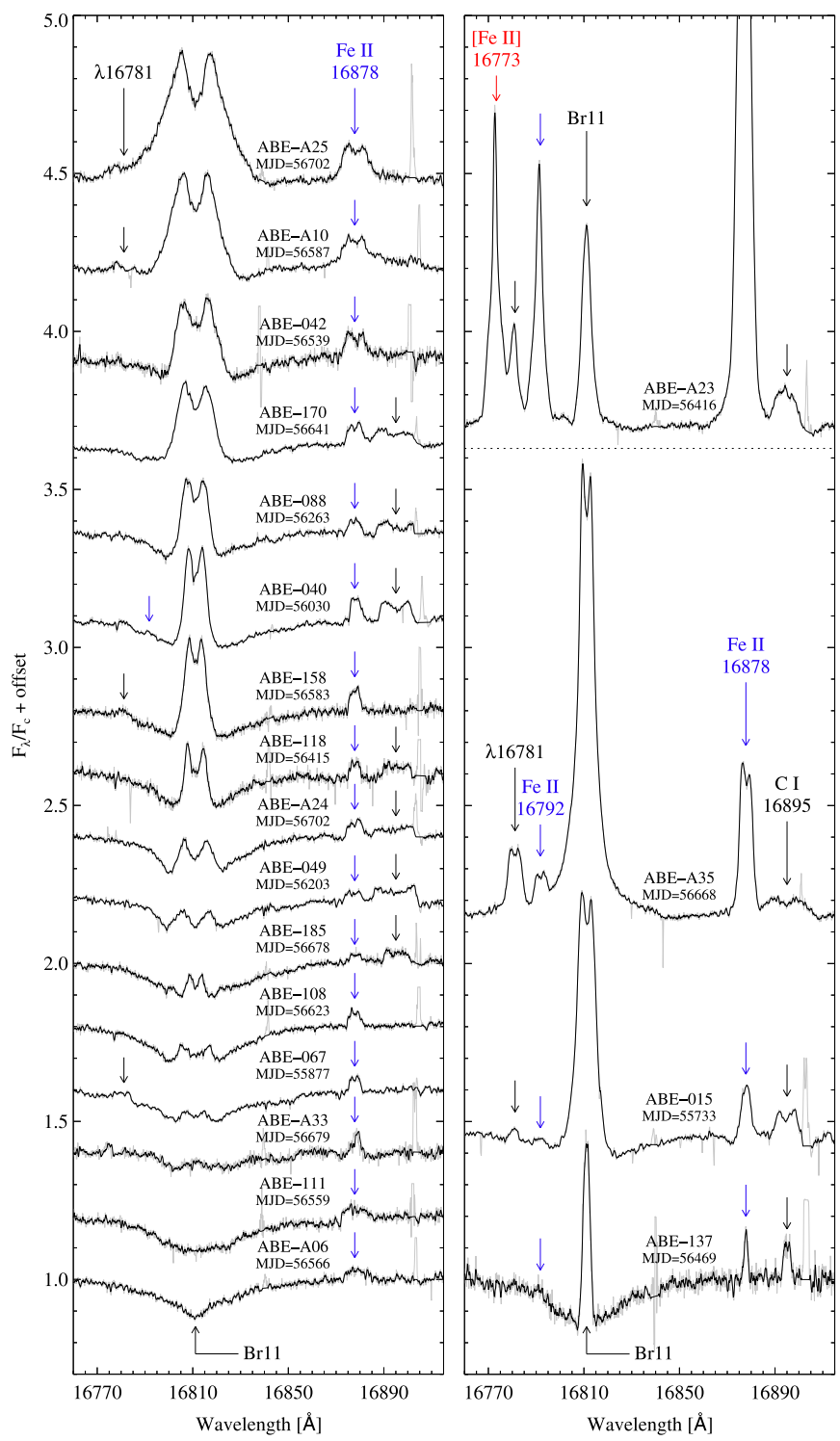

Figure 5. Be star spectra with combinations of emission from $\lambda 16781$, Fe 16792 and 16878 (blue), and $C_{\text {I }} 16895$ over a spectrum of Brackett series emission strength. The left and right panels show the same wavelength and intensity ranges. A dotted line separates the unclassified B[e] star ABE-A23 (MWC 922) from the other stars; ABE-A23 is unique among this sample (see Section 3.5) in being the only source to show forbidden line emission (mostly $[\mathrm{Fe} \mathrm{II}]$ ).

suggesting that NIR $\mathrm{C}_{\mathrm{I}}$ emission is ubiquitous across a wide range of evolutionary states.

\subsubsection{I-strong Be Stars}

Abnormally strong $C_{\text {I }} 16895$ emission is accompanied by weaker, similarly profiled emission lines from neutral and singly-ionized species in the spectra for at least five $\mathrm{ABE}$ stars. Figure 7 displays full APOGEE spectra for ABE-A15, ABE-188, and ABE-084, ABE-031, and ABE-004, the best examples of this marked deviation from the typical $H$-band emission line content for Be stars. The $\mathrm{C}_{\text {I }} 16895$ emission is blended with $\mathrm{Fe}$ II 16878 for ABE-A15 and possibly also for ABE-188. Two other $\mathrm{C}_{\mathrm{I}}$ lines at $16009.27 \AA$ and $16026.08 \AA$ are blended in emission for these stars, leading us to refer to the group as " $\mathrm{C}$ I-strong" Be stars. 

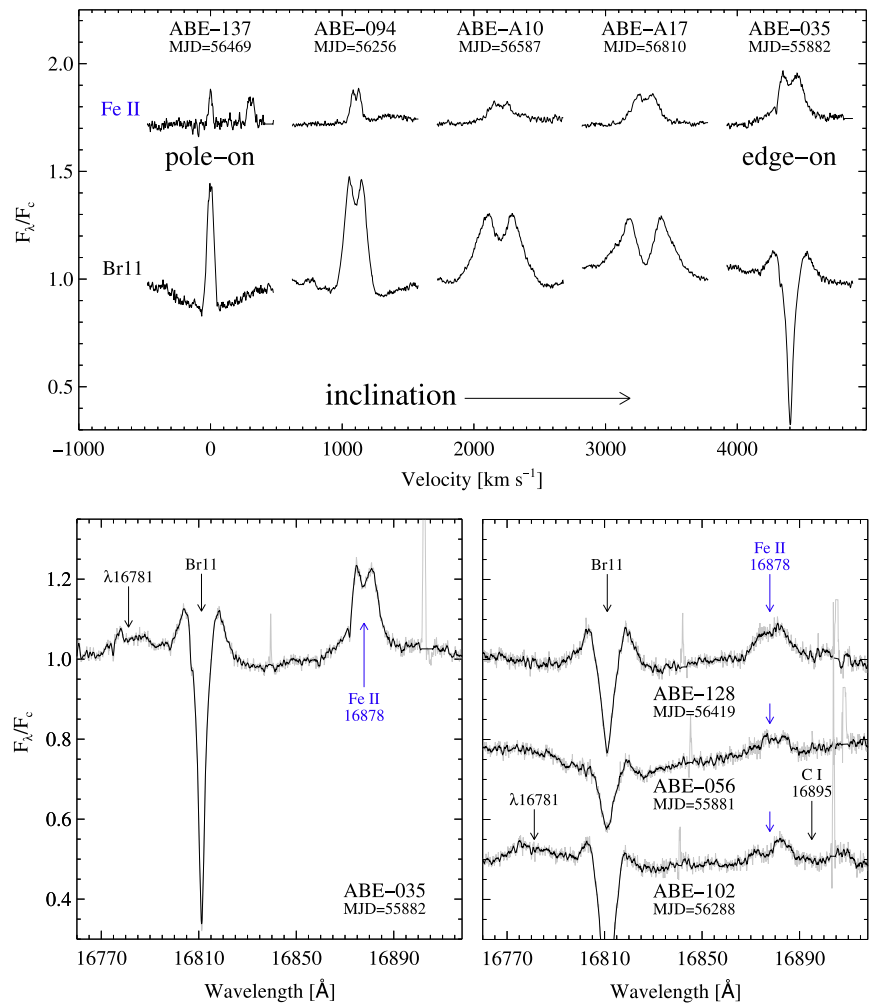

Figure 6. (Top panel) An assortment of observed spectra, showing the variety of metallic and hydrogen line profiles observed in the sample as a function of inclination angle. (Left, bottom) A portion of a spectrum for ABE-035, highlighting the immunity of metallic emission lines to shell absorption, a fact that is observed in all shell absorption sources (examples shown on right, bottom).

Most of the metallic emission features for the $\mathrm{C}$ I-strong $\mathrm{Be}$ stars correspond to strong absorption lines for late-A and cooler stars. An APOGEE spectrum for HD 163271, which is either a single metallic-line A star (A2/A3m) or the superposition (A2/ $\mathrm{A} 3+\mathrm{F} 0$ ) of an A star with an F star (Houk \& SmithMoore 1988; Renson et al. 1991), is provided in Figure 7 to demonstrate the typical line content for A-F stars. Small blue line segments indicate the numerous strong $\mathrm{Fe}_{\mathrm{I}}$ lines covered, with Fe 15299 being the strongest and appearing in emission for the Be stars. It is likely that emission from other Fe I lines is involved in much of the blending in the $\mathrm{C}$ I-strong $\mathrm{Be}$ star spectra.

Detections of resolved emission peaks for the $\mathrm{S}_{\mathrm{I}}$ lines labeled in Figure 7 are unavailable, but the lines may contribute to the weak bumps and blending around Br17. The transition probability measures for these $S_{\text {I lines suggests } S_{\text {I }}}$ 15426 should be the strongest of them and the A star spectrum appears to confirm this. Since the Br17 profiles for the $\mathrm{C}$ I-strong Be stars do not appear distorted by significant underlying emission from other lines however, it is unclear whether the $\mathrm{S}_{\mathrm{I}}$ lines are actually observed as emission features.

The strong emission lines redward of Br15 are due partly to several $\mathrm{Mg}$ I lines, with the strongest contributions being Mg I 15753.291 and Mg I 15770.149. The Mg I lines are also seen weakly in emission and unblended for ABE-149; all of the emission lines are single-peaked for that source, including $\mathrm{HI}, \mathrm{Fe}$ II 16878, C I 16895, and the Mg I lines (see the Appendix). Above the ABE-A15 spectrum in Figure 7 is a small panel that zooms in on the $\mathrm{Mg}$ I blend for $\mathrm{ABE}-\mathrm{A} 15$, demonstrating that emission from $\lambda 15760$ is also a major contributing factor in the blend. Black arrows in the small panel point out the sharp $\lambda 15760$ peaks that mimic the sharp $\lambda 16781$ peaks. ABE-004 similarly has the $\lambda 15760$ and $\lambda 16781$ lines clearly in emission.

As for the line around $15964 \AA$, PLL suggests two possible identities: Cl I 15964.11 and Si I 15964.4218. Since other covered $\mathrm{Cl}$ I lines are expected to be stronger than $\mathrm{Cl}_{\text {I }} 15964.11$ are covered but do not appear in emission (e.g., Cl I 15524.70), $\mathrm{Si}$ I seems the more likely to cause the $15964 \AA$ emission. The line blended with Br14 (most noticeable for ABE-084 and ABE-031) is suspected to be $\mathrm{Si}$ I 15892.7713, the next strongest Si I line covered after Si I 15964.4218.

The weak double-peaked line around $\sim 16565 \AA$ is possibly $\mathrm{Ca}$ II 16565.59 , but the ambiguous detection of $\mathrm{Ca}$ II 16654.43 calls the $\mathrm{Ca}$ II identification into question since the latter line should be stronger. On the other hand, the position of $\mathrm{Ca}$ II 16654.43 corresponds to a strong telluric band which is poorlycorrected and may cause the ambiguity. Emission from the Ca II triplet $(8498,8542,8662 \AA)$ is observed for some Be stars (Hiltner 1947; Polidan \& Peters 1976), so $H$-band Ca II emission would not be terribly unexpected. A $\mathrm{C}_{\mathrm{I}}$ line at 16564.13 A probably does not contribute since similar C I lines, covered and expected to be stronger than $\mathrm{C}_{\mathrm{I}} 16564$, fail to appear.

The cause of the strong $\mathrm{C}_{\mathrm{I}} 16895$ in addition to other weaker emission lines for the $\mathrm{C}_{\mathrm{I}}$-strong Be stars remains unknown. Based on the available examples however, such as ABE-031 where the weak emission features persist in 12 spectra covering 1.2 years, the phenomenon appears to be permanent rather than a particular stage of short- or mediumterm intrinsic variability.

\section{5. $\lambda 15760$ and $\lambda 16781$}

The $\lambda 15760$ and $\lambda 16781$ emission lines discussed by Steele $\&$ Clark (2001) are present for between 15-21\%. As is demonstrated in Figure 8, these lines always appear together with matching intensity and $\mathrm{V} / \mathrm{R}$ orientation. In the available examples where peak separations were measurable for $\lambda 15760$ and $\lambda 16781$, those values are nearly identical as well (see Section 5.2). Fe II 16878 is usually detected in unison with $\lambda 15760$ and $\lambda 16781$, but this is not a strict rule. Non-detection of Fe II 16878 is accompanied by detections $\lambda 15760$ and $\lambda 16781$ for ABE-180, ABE-A05, and ABE-005, the three lower-most stars represented in Figure 8.

The $\lambda 15760$ line has been identified as Fe II in several past papers (Steele \& Clark 2001; Smith 2001; Kraus et al. 2012). In a study of $\eta$ Carinae, Hamann et al. (1994) was apparently the first to note proximity of $\lambda 15760$ to an Fe II transition. The authors of that paper appended a question mark to the Fe II identification listed in an emission line table, but it seems that over the years the question mark was forgotten. PLL lists an Fe II line at $15761.78 \AA$, but no indication of expected transition strength is available. NIST provides wavelength for different Fe II transitions, at 15759.720 and $15760.563 \AA$, with the lower energy levels again more than doubling those of Fe II 16878 (5.5 eV versus $13.4 \mathrm{eV}$ ) and again lacking transition strength indication. A firm identification for this emission line remains elusive.

Whereas Steele \& Clark (2001) restricted the possible identifications for $\lambda 16781$ to [Fe II] 16773 and Fe II 16792, the much higher-resolution APOGEE spectra rule out both of 


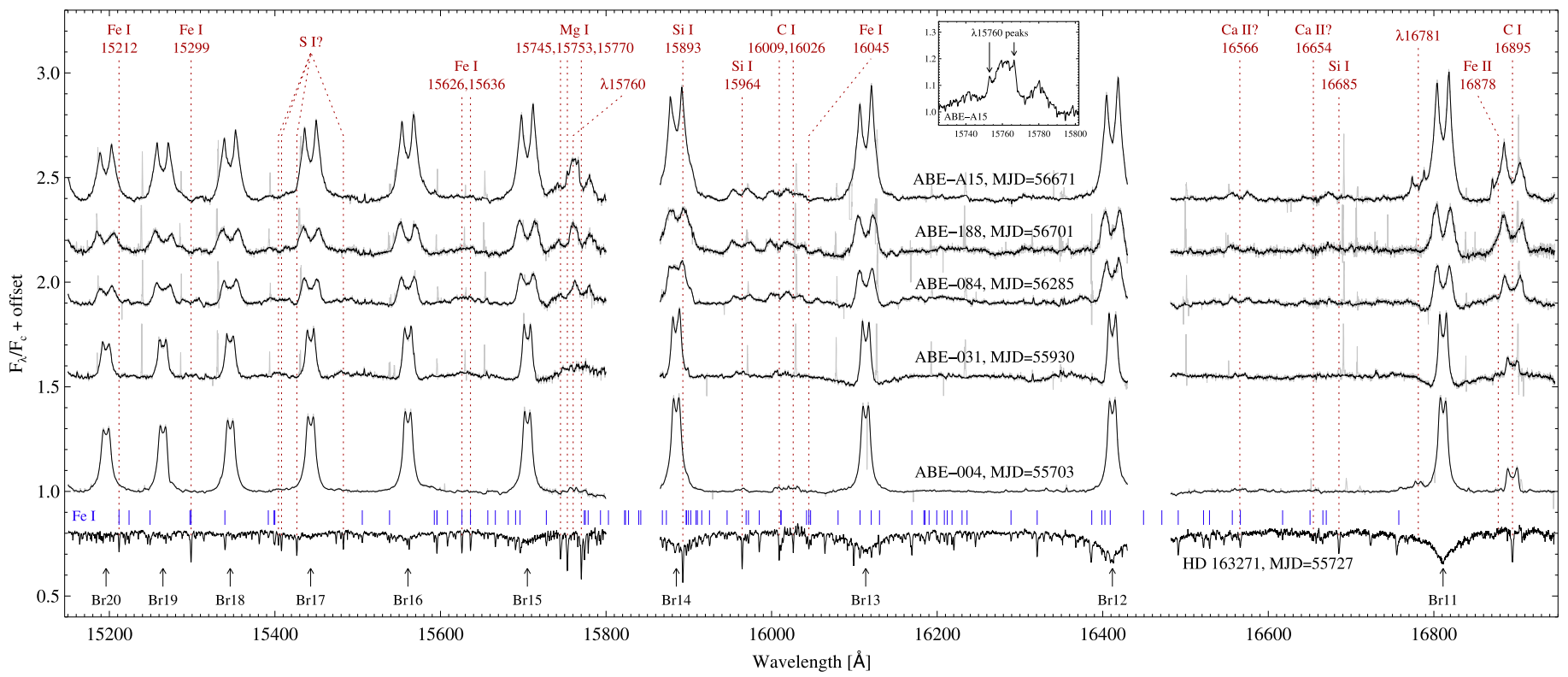

Figure 7. Spectra of five Be stars (ABE-A15, ABE-188, ABE-084, ABE-031, ABE-004) with strong C 16895 emission and many weak, double-peaked metallic emission features. The spectrum of a strong-metal-lined A star (HD 163271) is included to demonstrate that the additional emission lines for these four Be stars correspond to absorption lines for cooler stars. The small lines (blue) above the A star spectrum mark the positions of numerous $\mathrm{Fe}$ I lines with $\log \left(g_{i} f_{i k}\right)>-3$.

those lines as possibilities (see ABE-A23 spectrum in Figure 5). PLL includes several He I lines around $16780 \AA$, but considering that an absorption line is never seen at this wavelength for normal OB stars, $\lambda 16781$ is probably not He I. Also listed in PLL is an O I multiplet at $16781.7 \AA$, lacking transition probability data and being quickly ruled out by non-detection of other $\mathrm{O}_{\mathrm{I}}$ lines covered and expected to be stronger. Through similar argument, other lines listed in PLL and NIST around $\lambda 16781$ are readily ruled out as possibilities.

Whatever the identities of $\lambda 15760$ and $\lambda 16781$, the features behave similarly to $\mathrm{Fe}$ II in being present as emission lines or not present at all: no corresponding absorption for features are seen for APOGEE-observed stars of any type. Reliable spectral types have been reported for $24 \mathrm{ABE}$ stars with $\lambda 15760$ and $\lambda 16781$ detections and 20/24 are B3 or hotter, so it is possible that $\lambda 15760$ and $\lambda 16781$ are relatively high-ionization lines. One possible example of $\lambda 16781$ being detected despite absence of $\lambda 15760$ is ABE-A36, a peculiar star discussed in Section 6.1. However, the bump in the $\mathrm{V}$ wing of Br11 for ABE-A36 is not sufficiently convincing to cause us to doubt that $\lambda 15760$ and $\lambda 16781$ should always be expected to appear simultaneously.

\section{6. $N_{I}$}

The expected strongest and second strongest $\mathrm{N}_{\mathrm{I}}$ lines covered are seen in emission for ABE-A35. Figure 9 shows a portion of a spectrum for ABE-A35 encompassing the $\mathrm{N}_{\mathrm{I}}$ 15586.545 and $\mathrm{N}_{\mathrm{I}}$ 15687.160 lines as well as Br16, Br15 and $\lambda 15760$. Neither of the $\mathrm{N}_{\mathrm{I}}$ lines are detected for any other objects beyond ABE-A35, but they are present in all five APOGEE spectra for ABE-A35. Although [Fe II] 15586.550 is coincident in position with the stronger $\mathrm{N}_{\mathrm{I}}$ line, it is far from the strongest $[\mathrm{Fe}$ II] feature covered. The lack of detection in the ABE-A35 spectra of the stronger $\left[\mathrm{Fe}_{\mathrm{II}}\right]$ lines rules those lines out as possibilities. Forbidden line emission in the optical was noted as early as 1976 for ABE-A35 (Allen \& Swings 1976), but in the $H$-band the only clues suggesting the $\mathrm{B}[\mathrm{e}]$ nature of this object are the abnormally strong $\mathrm{HI}$, Fe II, and Fe II-like emission lines.

\section{7. $M g$ II and Si II}

The lowest-energy $\mathrm{Mg}$ II and $\mathrm{Si}$ iI lines covered in APOGEE data appear clearly in emission for ABE-A26 and are not confidently detected for any of the other stars. ABE-004, ABEA05, and ABE-A29 possibly show exceedingly weak contributions from these lines, but blending renders the situation ambiguous in all cases aside from ABE-A26.

Figure 10 displays a spectrum for ABE-A26 over differing wavelength ranges: the upper panel shows the full spectrum, while the middle and lower panels focus on the weak emission lines around Br11. Identification of the line blueward of $\lambda 16781$ as $\mathrm{Mg}$ II 16764.80 requires that the stronger of three lines comprising this $\mathrm{Mg}$ II multiplet also be detected, and indeed the lower panel of Figure 10 shows that Mg II 16804.52 is visible in the $\mathrm{V}$ wing of $\mathrm{Br} 11$ at apparently the correct intensity relative to $\mathrm{Mg}$ II 16764.80 . Based on the intensities of these lines, the third Mg II line of the multiplet $(16803.67 \AA)$ is not expected to appear and would overlap with Mg II 16804 anyway. The weak emission line redward of Fe II 16878 in the middle panel of Figure 10 is identified as Si II 16911.430, the strongest $\mathrm{Si}$ II line covered and a line with very similar energy levels to the $\mathrm{Mg}$ II lines (see Table 2).

In addition to detection of the relatively high-ionization $\mathrm{Mg}$ II and $\mathrm{Si}$ II lines, the combination of single-peaked Brackett series lines and double-peaked metallic lines is unique to ABE-A26 among this sample. The double-peaked lines indicate that at least some of the circumstellar gas is organized in a disk. It is possible that ABE-A26 was observed by APOGEE during of after an outburst such that substantial Brackett series emitting gas is in the polar regions, leading to the single-peaked Brackett lines. 

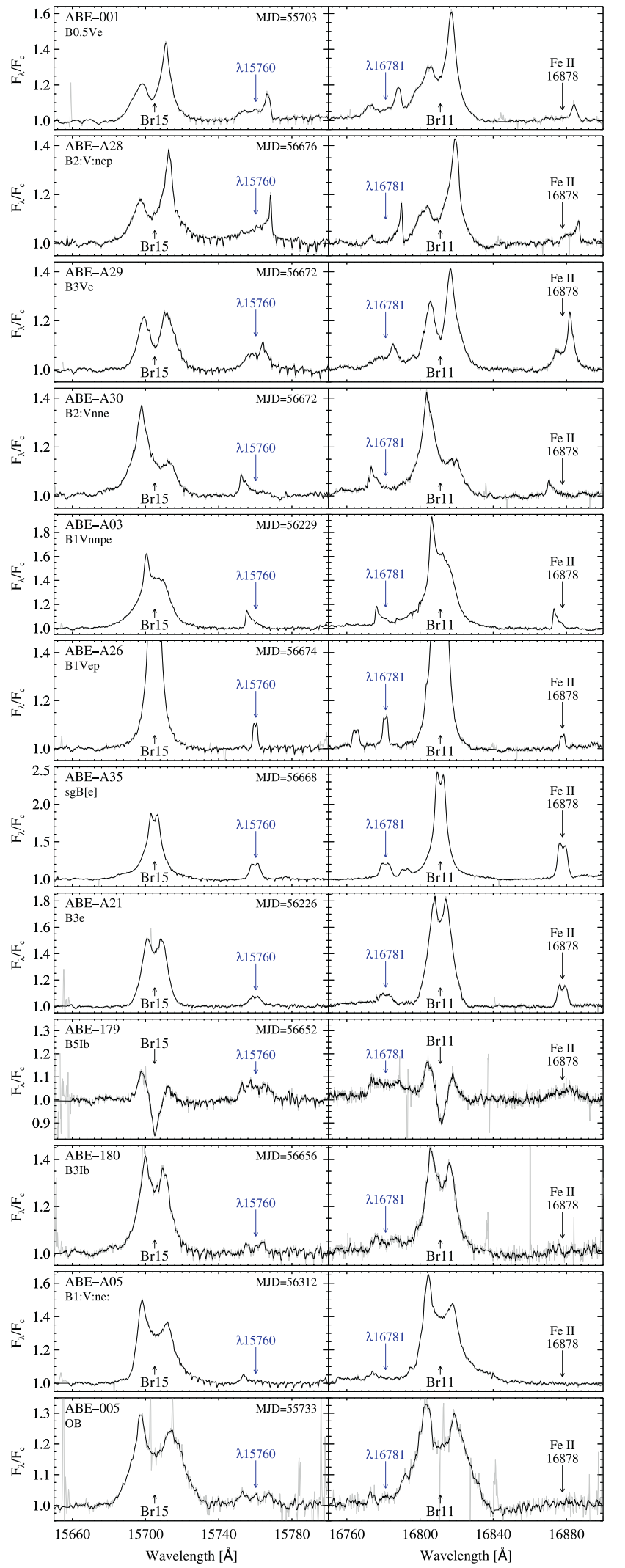

Figure 8. Identifications of $\lambda 15760$ and $\lambda 16781$ are uncertain; however, these lines are never detected separately and in most cases Fe II 16878 emission is also present. The three lines always share a common V/R orientation, but the $\mathrm{Fe}$ II intensity varies with respect to $\lambda 15760$ and $\lambda 16781$. Small absorptions around the $\lambda 15760$ line are telluric correction artifacts.

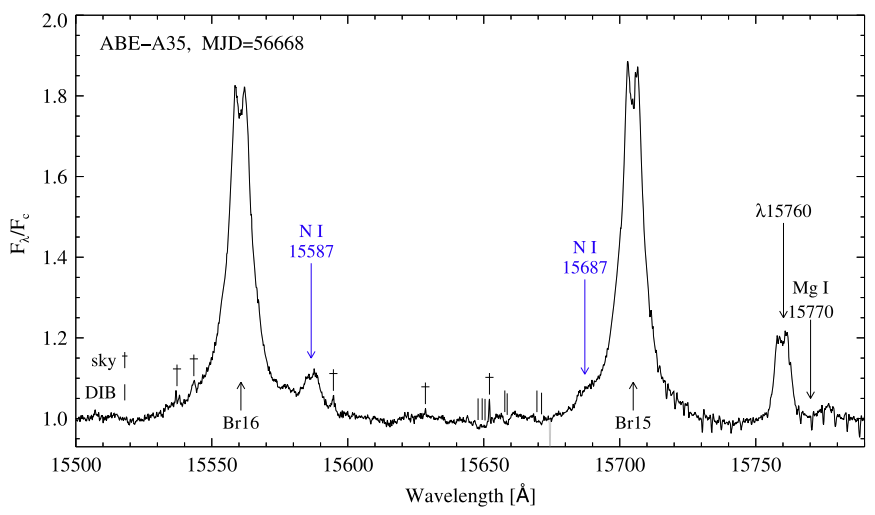

Figure 9. Emission from $\mathrm{N}_{\mathrm{I}}$ is seen only for $\mathrm{ABE}-\mathrm{A} 35$, a supergiant $\mathrm{B}[\mathrm{e}]$ star (Esteban \& Fernandez 1998; Oksala et al. 2013). Both lines, N I 15587 and N I 15687 , are partially blended with $\mathrm{H}$ I emission wings.

\section{PEAK SEPARATIONS}

\subsection{Stars with Abnormally Large $\Delta v_{\mathrm{p}}$}

Optical spectroscopy revealed that two stars, ABE-050 (HD 345439) and ABE-075 (HD 23478), with extremely large Brackett emission widths and double-peak separations are not classical Be stars (Eikenberry et al. 2014). Rather, these stars are analogues to the prototype magnetic emission B star $\sigma$ Orionis E first described as "helium-rich" by Greenstein \& Wallerstein (1958) and subsequently providing the first application (Townsend et al. 2005) of the Rigidly Rotating Magnetosphere model of Townsend \& Owocki (2005). Large $\Delta v_{p}$ for the Brackett series emission was a clue suggestive of a non-classical nature for these stars, but confirmation lay in the fact that both stars exhibit $\mathrm{HI}_{\mathrm{I}}$ emission well beyond the projected $v \sin i$ values (in these cases, a factor of two ore more beyond the projected $v \sin i$ ). For classical Be stars with Keplerian disks, the velocity separations of emission peaks do not exceed $2 v \sin i$ (Dachs et al. 1992).

Figure 11 compares the Br11 profiles of ABE-050 and ABE075 to the stars with the next largest peak separations. Arrows indicate the measured peak separations and for ABE- 050 and ABE-075, the inner sets of arrows indicate the $v \sin i$ values from Eikenberry et al. (2014). As there are only a handful of magnetic $\mathrm{B}$ emission stars known to exist, it seems more likely that the ABE-155, ABE-168, ABE-124, and ABE-099 are weak-disked, edge-on classical Be stars rather than additional $\sigma$ Orionis E types. Either way, optical follow-up spectroscopy is required for proper diagnosis.

\subsection{Line-by-line $\Delta v_{\mathrm{p}}$ Comparison}

Figure 12 plots the peak separations for Br11 versus the peak separations for Br12-Br18, Br20, $\lambda 16781, \lambda 15760$, Fe II 16878, Fe II 16792, C I 16895, Si I 15964, and Mg I 15770 . Each point represents the average peak separation for a line, from all spectra for a given star in which the $\operatorname{Br} 11 \Delta v_{p}$ was measured in addition to the $\Delta v_{\mathrm{p}}$ of the line represented on the $y$-axis. In the upper nine panels, plus signs (red) correspond to stars for which the Br20 peak separation was measured and therefore to stars with strong or particularly sharp-peaked emission. The 

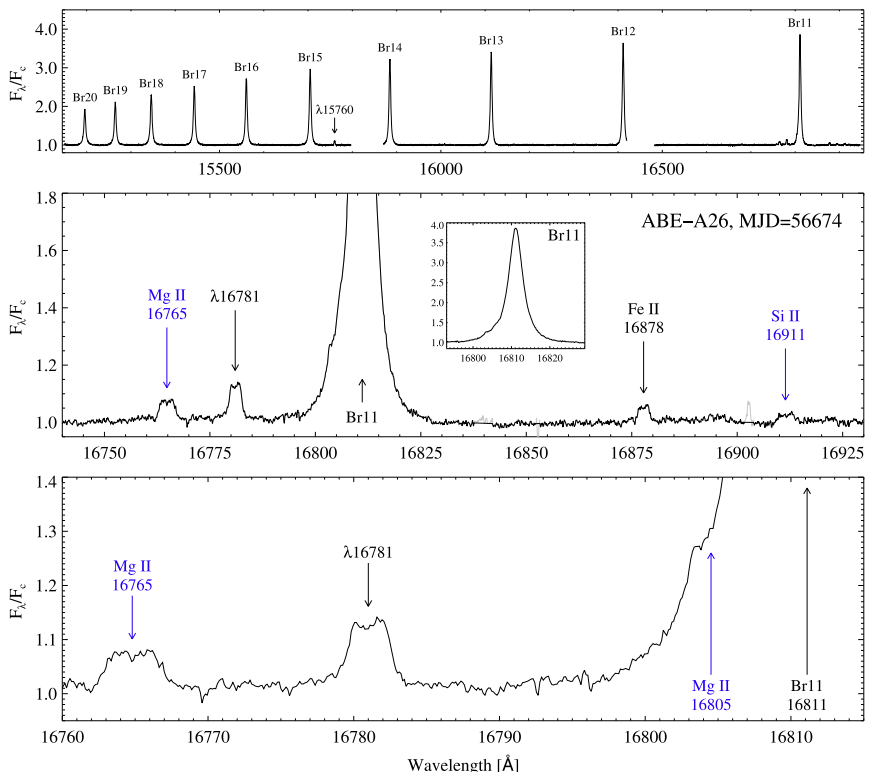

Figure 10. $\mathrm{Mg}$ II and $\mathrm{Si}$ II emission lines in a spectrum of ABE-A26, the only star for which these lines are detected. While the full spectrum is presented in the upper panel, the lower two panels highlight the Br11 region and the weak metallic lines therein. The single-peaked Br11 line profile is displayed in the inset panel of the larger middle panel for comparison to the double-peaked profiles of the metallic lines. As expected from the detection of $\mathrm{Mg}$ II 16765, the stronger line of this multiplet, Mg II 16804, appears blended with Br11 in the lowermost panel.

gaps between $\sim 400-500 \mathrm{~km} \mathrm{~s}^{-1}$ in the Br11 versus Br18 and Br11 versus Br17 panels are due to strong airglow lines impacting the emission peaks at large line width. High velocity gaps in the Br11 versus Br12 and Br11 versus Br14 panels are caused by either the $\operatorname{Br} 12 \mathrm{~V}$ peak or the $\mathrm{Br} 14 \mathrm{R}$ peak falling too close to gaps between detectors. For Br13 and Br15, telluric absorption contamination is more likely for large line width. Grey lines indicate 1-to-1 relationships between the lines plotted in each panel.

The effect of increasing $\Delta v_{\mathrm{p}}$ toward weaker $\mathrm{H}_{\mathrm{I}}$ lines is well-known for the Balmer (Hanuschik et al. 1988) and Paschen (Andrillat et al. 1990) lines, and the Brackett series lines are not an exception. Some stars (primarily the narrowlined variety with $\operatorname{Br} 11 \Delta v_{\mathrm{p}}<200 \mathrm{~km} \mathrm{~s}^{-1}$ ) show very little or no variation among Brackett series $\Delta v_{\mathrm{p}}$ but no convincing examples are found of decreasing $\Delta v_{\mathrm{p}}$ from Br11 toward $\mathrm{Br} 20$. We interpret the increasing peak separations toward weaker lines as kinematic in nature, such that the weaker Brackett lines $(\mathrm{Br} 12-20)$ are simply formed closer to the rapidly-rotating central stars than e.g., Br11. The Br11-20 lines never take the form of winebottle-type profiles frequently observed in the optically thick $\mathrm{H} \alpha$ line, where the effect of non-coherent scattering can produce inflections in the emission profile and effectively reduce the observed peak separation (Hummel \& Dachs 1992). Section 4 of Hanuschik et al. (1996) provides a summary of the line broadening factors that contribute to $\mathrm{Be}$ star emission line profile shapes.

Based on the lower three panels of Figure 12, when the $\Delta v_{p}$ of $\lambda 15760$ and $\lambda 16781$ are measured simultaneously, very similar values are found. The $\Delta v_{\mathrm{p}}$ for these lines are usually slightly smaller than the $\operatorname{Br} 11 \Delta v_{p}$ but can be slightly larger as

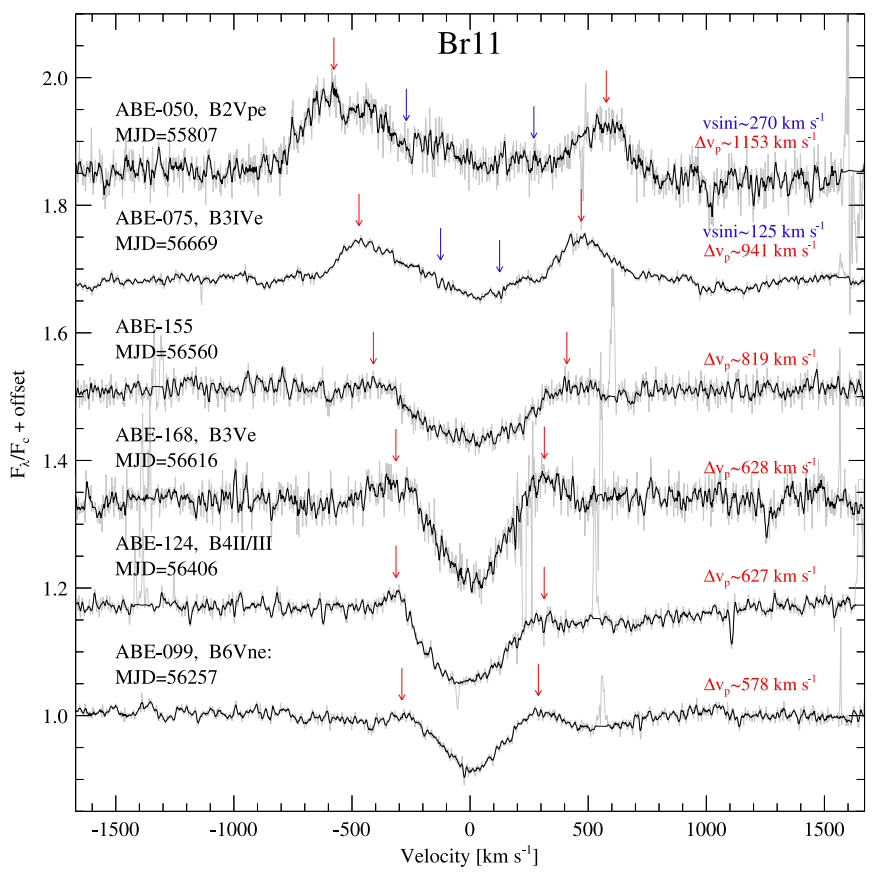

Figure 11. Br11 line profiles for the ABE stars with the largest peak separations are shown. The $\Delta v_{\mathrm{p}}$ is listed and marked with arrows (red) for each source, while the $v$ sin $i$ measurements for ABE-050 and ABE-075 are given and indicated with arrows (blue) interior to the $\Delta v_{\mathrm{p}}$ arrows. Whereas ABE-050 and ABE-075 are confirmed $\sigma$ Orionis E type stars, the other four stars remain to be investigated further.

well. Excluding ABE-A26, for which Br11 is single-peaked, $\mathrm{Fe}$ II 16878 is a strictly small- $\Delta v_{\mathrm{p}}$ line relative to Br11 with all $\Delta v_{\mathrm{p}}$ measurements less than $140 \mathrm{~km} \mathrm{~s}^{-1}$. The $\mathrm{C}_{\text {I }} 16895 \mathrm{Si}$ I 15964 , and $\mathrm{Mg}$ I 15770 lines share nearly identical $\Delta v_{\mathrm{p}}$ in the available examples (ABE-084, ABE-188, ABE-A15; see Figure 7), and all of the $\Delta v_{p}$ measurements for $C_{\text {I }} 16895$ exceed the $\operatorname{Br} 11 \Delta v_{p}$.

\subsection{Line-emitting Disk Radii}

For Keplerian rotation in a gaseous disk, the orbital velocity decreases according to $r^{-1 / 2}$, where $r$ is the radial distance from star to disk. Given knowledge of the stellar rotational velocity, $v \sin i$, the peak separation of an emission line can be used to calculate the approximate outer radius in the disk, $r_{\mathrm{d}}$, at which that line is preferentially formed (Smak 1969; Huang 1972; Smak 1981; Horne \& Marsh 1986). Many authors (e.g., Hanuschik 1987; Hanuschik et al. 1988; Andrillat et al. 1990; Dachs et al. 1992; Slettebak et al. 1992) have used this relation (Huang's law) to study the geometry of Be disks by estimating the individual line-emitting radii for $\mathrm{H}$ I and metallic lines in the optical region. In units of stellar radii, $R_{*}, r_{\mathrm{d}}$ is calculated via Huang's Law (Huang 1972) as

$$
r_{\mathrm{d}}=\left(\frac{2 \times v \sin i}{\Delta v_{\mathrm{p}}}\right)^{2}
$$

where the Equation is squared due to the assumption of a circular orbit. The resulting outer disk radii measurements for the subset of stars with available $v \sin i$ from the literature and Br11 or metallic line $\Delta v_{\mathrm{p}}$ measurements are listed in Table 3. In estimating $r_{\mathrm{d}}$, the average $\Delta v_{\mathrm{p}}$ measured from all spectra for 


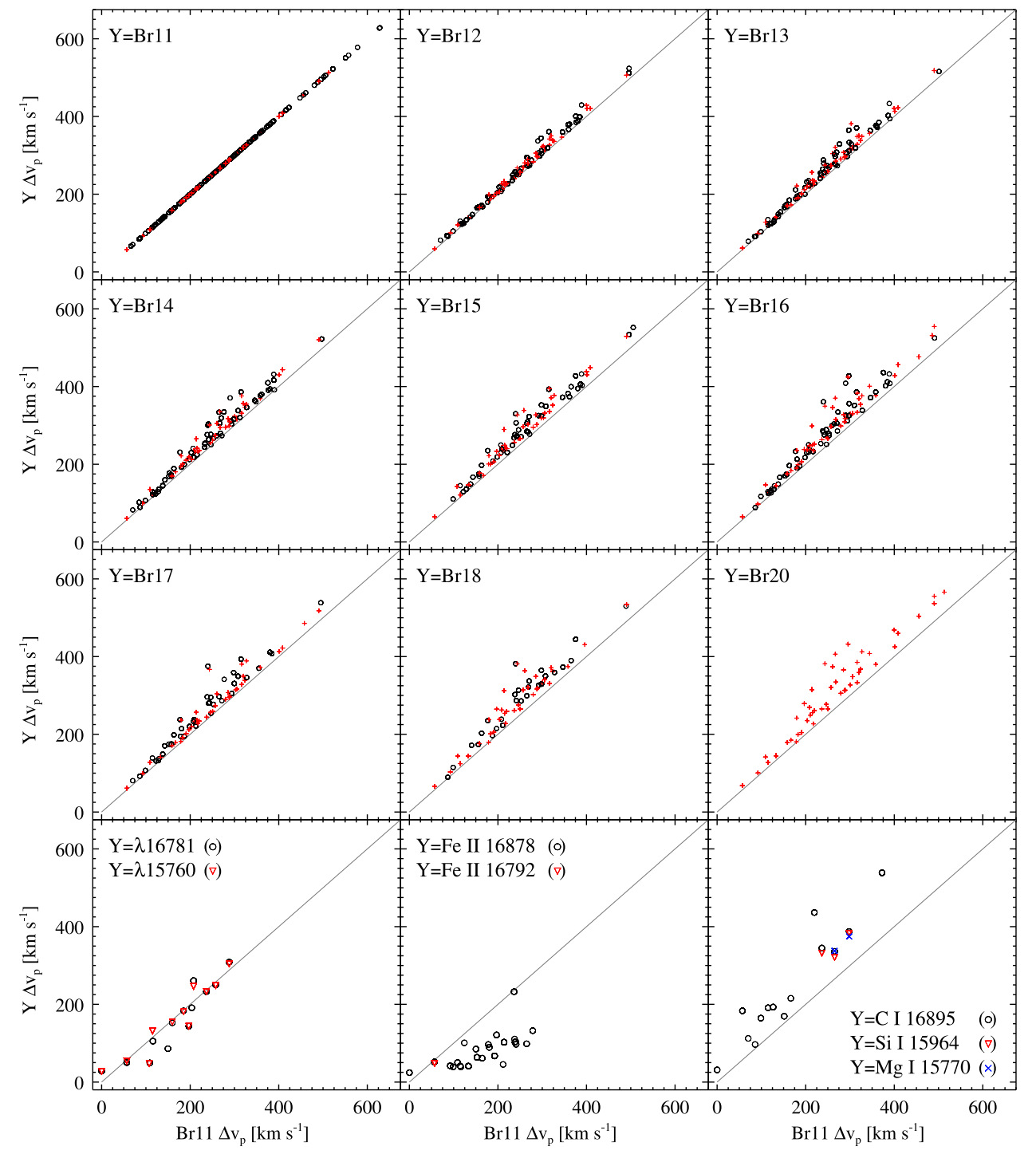

Figure 12. Peak separation of Br11 is compared to the peak separations for the other Brackett series lines as well as the most routinely detected metallic emission lines. Symbol meanings are described in Section 5.2.

each star (the number of spectra used for each star is indicated in the "\# Obs" column) have been used. The relation in Equation (1) may not necessarily hold for cases of emission lines with asymmetric peak intensities or for shell profiles so these instances have been noted in Table 3. In particular, large $r_{\mathrm{d}}$ estimates $\left(r_{\mathrm{d}}>5 r_{*}\right)$ correspond to asymmetric and shell profiles.

Taking the average of the $\operatorname{Br} 11 r_{\mathrm{d}}$ estimates for the 19 nonshell stars with roughly symmetric emission peaks, we find an average Br11 formation outer radius and associated standard deviation of

$$
r_{\mathrm{d}}(\mathrm{Br} 11)=2.21 \pm 0.73 R_{*}
$$

Hanuschik et al. (1988) found an average $\mathrm{H} \alpha$-emitting radius of $\sim 20 R_{*}$, while Slettebak et al. (1992) found an average of $\sim 19 R_{*}$. It is important to note, however, that results from interferometry confirm that application of Huang's law to the double peaks of winebottle-type profiles (appearing for optically thick lines like $\mathrm{H} \alpha$ ) leads to artificially large disk radii estimates (Hummel \& Dachs 1992). Interferometric studies typically produce radii estimates of less then $10 R_{*}$ over the optical and $J H K$ bands (see disk radius measurements and papers referenced in Table 2 of (see disk radius measurements and papers referenced in Table 2 of Rivinius et al. 2013b), more similar to what is found here from the Brackett lines.

For $\mathrm{H} \gamma$ and $\mathrm{Fe}$ II $6516 \AA$, Slettebak et al. (1992) found emitting radii of $\sim 7.4 R_{*}$ and $\sim 3.9 R_{*}$ respectively. In a study of optical Fe II emission lines for Be stars, Arias et al. (2006) found that, on average, the optical $\mathrm{Fe}$ II lines are formed at an outer disk radius of 2.0 stellar radii. Therefore, the Br11emitting outer radius is roughly coincident with the optical $\mathrm{Fe}$ II-emitting outer radius and well inside the $\mathrm{H} \alpha$-emitting radius.

Given the sparsity and potential wide-range of quality of $v \sin i$ information for our sample, disk radii are only estimated for the Br11 and metallic lines. However, it follows from Figure 12 that the $\mathrm{Br} 12-\mathrm{Br} 20$ formation outer radii are interior to that of Br11. Andrillat et al. (1990) and Slettebak et al. (1992) found a correlation between formation location of individual optical lines and the upper energy levels $\left(E_{k}\right)$ of the lines. Weaker lines with higher $E_{k}$ were generally found to have larger $\Delta v_{\mathrm{p}}$ and hence smaller $r_{\mathrm{d}}$. This is also the case for the 
Table 3

Line-emitting Disk Radius Estimates

\begin{tabular}{|c|c|c|c|c|c|c|}
\hline $\begin{array}{l}\text { ABE } \\
\text { ID }\end{array}$ & $\begin{array}{c}\text { Lit. } \\
v \sin i \\
\left(\mathrm{~km} \mathrm{~s}^{-1}\right)\end{array}$ & Ref. & $\begin{array}{l}\text { Atom } \\
\text { or } \\
\text { Ion }\end{array}$ & $\begin{array}{c}\# \\
\text { spectra }\end{array}$ & $\begin{array}{c}\text { Mean } \\
\Delta v_{\mathrm{p}} \\
\left(\mathrm{km} \mathrm{s}^{-1}\right)\end{array}$ & $\begin{array}{c}\text { Mean } \\
r_{\mathrm{d}} \\
\left(R_{*}\right) \\
\end{array}$ \\
\hline \multirow[t]{3}{*}{$001^{\mathrm{a}}$} & 266 & 9 & Br11 & 4 & 208 & 6.56 \\
\hline & & & $\lambda 15760$ & 4 & 246 & 4.67 \\
\hline & & & $\lambda 16781$ & 4 & 261 & 4.16 \\
\hline 003 & 225 & 5 & Br11 & 10 & 305 & 2.18 \\
\hline 006 & 231 & 7 & Br11 & 3 & 294 & 2.47 \\
\hline 014 & 230 & 3 & Br11 & 7 & 326 & 1.99 \\
\hline \multirow[t]{2}{*}{$016^{\mathrm{a}}$} & 250 & 4 & Br11 & 1 & 238 & 4.43 \\
\hline & & & Fe II 16878 & 1 & 111 & 20.41 \\
\hline $026^{\mathrm{b}}$ & 230 & 3 & Br11 & 12 & 324 & 2.02 \\
\hline 028 & 242 & 4 & Br11 & 12 & 297 & 2.66 \\
\hline $036^{\mathrm{b}}$ & 307 & 4 & Br11 & 3 & 456 & 1.81 \\
\hline $046^{\mathrm{b}}$ & 235 & 2 & Br11 & 1 & 294 & 2.56 \\
\hline \multirow[t]{2}{*}{049} & 120 & 2 & Br11 & 23 & 197 & 1.48 \\
\hline & & & Fe II 16878 & 7 & 67 & 12.73 \\
\hline \multirow[t]{2}{*}{067} & 120 & 5 & Br11 & 8 & 133 & 3.24 \\
\hline & & & Fe II 16878 & 8 & 41 & 34.32 \\
\hline 085 & 182 & 8 & Br11 & 4 & 321 & 1.29 \\
\hline 098 & 166 & 11 & Br11 & 1 & 301 & 1.21 \\
\hline $133^{b}$ & 286 & 4 & Br11 & 3 & 285 & 4.02 \\
\hline 138 & 243 & 7 & Br11 & 2 & 365 & 1.77 \\
\hline 140 & 328 & 4 & Br11 & 4 & 382 & 2.95 \\
\hline 165 & 274 & 4 & Br11 & 3 & 397 & 1.91 \\
\hline 167 & 160 & 7 & Br11 & 3 & 300 & 1.14 \\
\hline \multirow[t]{3}{*}{170} & 130 & 5 & Br11 & 6 & 153 & 2.90 \\
\hline & & & Fe II 16878 & 6 & 64 & 16.76 \\
\hline & & & C I 16895 & 2 & 169 & 2.36 \\
\hline 189 & 256 & 6 & Br11 & 5 & 305 & 2.81 \\
\hline 191 & 242 & 4 & Br11 & 1 & 274 & 3.11 \\
\hline $195^{\mathrm{b}}$ & 148 & 7 & Br11 & 3 & 266 & 1.24 \\
\hline A08 & 343 & 11 & Br11 & 3 & 409 & 2.82 \\
\hline A11 & 220 & 7 & Br11 & 3 & 215 & 4.19 \\
\hline A12 & 215 & 7 & Br11 & 1 & 358 & 1.44 \\
\hline \multirow[t]{3}{*}{$\mathrm{A} 13^{\mathrm{a} b}$} & 306 & 4 & Br11 & 6 & 257 & 5.68 \\
\hline & & & $\lambda 15760$ & 4 & 248 & 6.07 \\
\hline & & & $\lambda 16781$ & 4 & 250 & 6.00 \\
\hline \multirow[t]{5}{*}{$\mathrm{A} 15^{\mathrm{a}}$} & 350 & 7 & Br11 & 3 & 237 & 8.76 \\
\hline & & & Fe II 16878 & 3 & 232 & 9.07 \\
\hline & & & C I 16895 & 3 & 345 & 4.12 \\
\hline & & & $\lambda 15760$ & 3 & 233 & 9.05 \\
\hline & & & $\lambda 16781$ & 3 & 233 & 9.04 \\
\hline \multirow[t]{2}{*}{$\mathrm{A} 17^{\mathrm{b}}$} & 300 & 1 & Br11 & 3 & 241 & 6.19 \\
\hline & & & Fe II 16878 & 3 & 102 & 34.32 \\
\hline A20 & 210 & 4 & Br11 & 3 & 358 & 1.37 \\
\hline \multirow[t]{4}{*}{$\mathrm{A} 29^{\mathrm{a}}$} & 222 & 4 & Br11 & 3 & 197 & 5.09 \\
\hline & & & Fe II 16878 & 3 & 121 & 13.42 \\
\hline & & & $\lambda 15760$ & 3 & 144 & 9.55 \\
\hline & & & $\lambda 16781$ & 3 & 144 & 9.52 \\
\hline A32 & 260 & 10 & Br11 & 3 & 380 & 1.87 \\
\hline
\end{tabular}

References. (1) Uesugi \& Fukuda (1970); (2) Uesugi \& Fukuda (1982); (3) Halbedel (1996); (4) Yudin (2001); (5) Abt et al. (2002); (6) Frémat et al. (2005); (7) Frémat et al. (2006); (8) Huang \& Gies (2006); (9) Bhavya et al. (2007); (10) Reig et al. (2010); (11) Huang et al. (2010).

a Asymmetric emission peak intensities.

${ }^{\mathrm{b}}$ Shell stars.

Brackett series lines, where $E_{k}$ increases slightly from $\mathrm{Br} 11$ to $\mathrm{Br} 20$.

A trend toward large $r_{\mathrm{d}}$ is evident for the Fe II 16878 line with respect to $\mathrm{Br} 11$. The average of the five available $r_{\mathrm{d}}$ estimates for $\mathrm{Fe}$ II is $\sim 19 R_{*}$, almost ten times the disk radius where $\mathrm{Br} 11$ is preferentially formed. A consequence of the widely varying formation radii between $\mathrm{Br} 11$ and $\mathrm{Fe}$ II 16878 is discussed in the following section.

\section{SINGLE-EPOCH VARIATION IN V/R AND RADIAL VELOCITY}

As outlined in Okazaki (1991), long-term V/R variability for Be stars often entails shifts in RV of entire emission line profiles toward whichever peak is stronger at the time and differences in V/R orientation between lines with different formation loci, such that $V / R$ is necessarily constant from atomic species to atomic species or from line to line. These effects are believed to be caused by perturbations with the disks that give rise to one-armed global density waves that slowly precess through the disk with periods averaging 7 years (Rivinius et al. 2013b).

Recent papers discussing the well known Be-shell star $\zeta$ Tau provided an example of V/R phase lags between Balmer and Brackett series lines and also between individual Brackett series lines. Wisniewski et al. (2007) hypothesized that the optical/NIR phase lag in V/R could be understood in terms of differing preferential formation radii and of the global density perturbation within the disk taking the form of a spiral arm. Stefl et al. (2009) and Carciofi et al. (2009) subsequently showed this to be the case.

\subsection{H I versus $H_{I}$ V/R Phase Lags}

Evidence of V/R phase lags within the Brackett series lines is present in the spectra for the $\mathrm{ABE}$ stars represented in Figure 13. Each Brackett line is displayed individually on a velocity scale in Figure 13 and, with the exception of ABEA36 (discussed below), the V/R of Br11 for each spectrum is printed in left-most Br11 panels while the differences between the $\mathrm{V} / \mathrm{R}$ of $\mathrm{Br} 11$ and the $\mathrm{V} / \mathrm{R}$ of $\mathrm{Br} 12-\mathrm{Br} 20$ are printed in the Br12-Br20 panels. For ABE-A29, the V/R orientations progress from $\mathrm{V}<\mathrm{R}$ at $\mathrm{Br} 11$ to $\mathrm{V} \simeq \mathrm{R}$ at $\mathrm{Br} 17$. The $\mathrm{Br} 19$ profile is contaminated by DIB 15271 absorption, but the Br18 and $\mathrm{Br} 20$ profiles have $\mathrm{V} \simeq \mathrm{R}$ similar to $\mathrm{Br} 17$. For $\mathrm{ABE}-\mathrm{A} 31$ and ABE-181 the opposite progression takes place as the $\mathrm{R}$ peak increases in dominance from $\mathrm{Br} 11$ to $\mathrm{Br} 20$. Although the Br11 profiles for ABE-A36, ABE-A31, and ABE-181 are contaminated by underlying metallic emission $(\lambda 16781$ and/or Fe II 16878), the V/R phase lag is nonetheless plainly visible from comparison of the $\mathrm{Br} 12$ or $\mathrm{Br} 13$ profiles to $\mathrm{Br} 20$.

Of the 238 stars comprising the ABE sample, ABE-A36 is the only available example of quasi-triple-peaked ( $q t p)$ Brackett series lines (see upper row of Figure 13), where the "quasi" implies ambiguity as to whether there is actually a true third emission peak or whether instead the central absorption is split into multiple components. The $\mathrm{V}$ emission peak of Br11 for ABE-A36 is slightly higher than the R peak due to possible blending with $\lambda 16781$, and the possible third peak appears between the dominant outer emission peaks with lesser intensity than those peaks. $\mathrm{Br} 12$ is similar in profile to $\mathrm{Br} 11$, but from $\mathrm{Br} 12-\mathrm{Br} 17$ the profiles gradually assume a "flat-topped" morphology with the apparent blue central absorption still weakly visible in contrast to the apparent red central absorption having all but disappeared. At $\mathrm{Br} 18$, evidence of the third (middle) emission peak emerges again, this time as the dominant peak since the outer $\mathrm{V}$ and $\mathrm{R}$ peaks have all but disappeared. $\mathrm{Br} 19$ is directly 

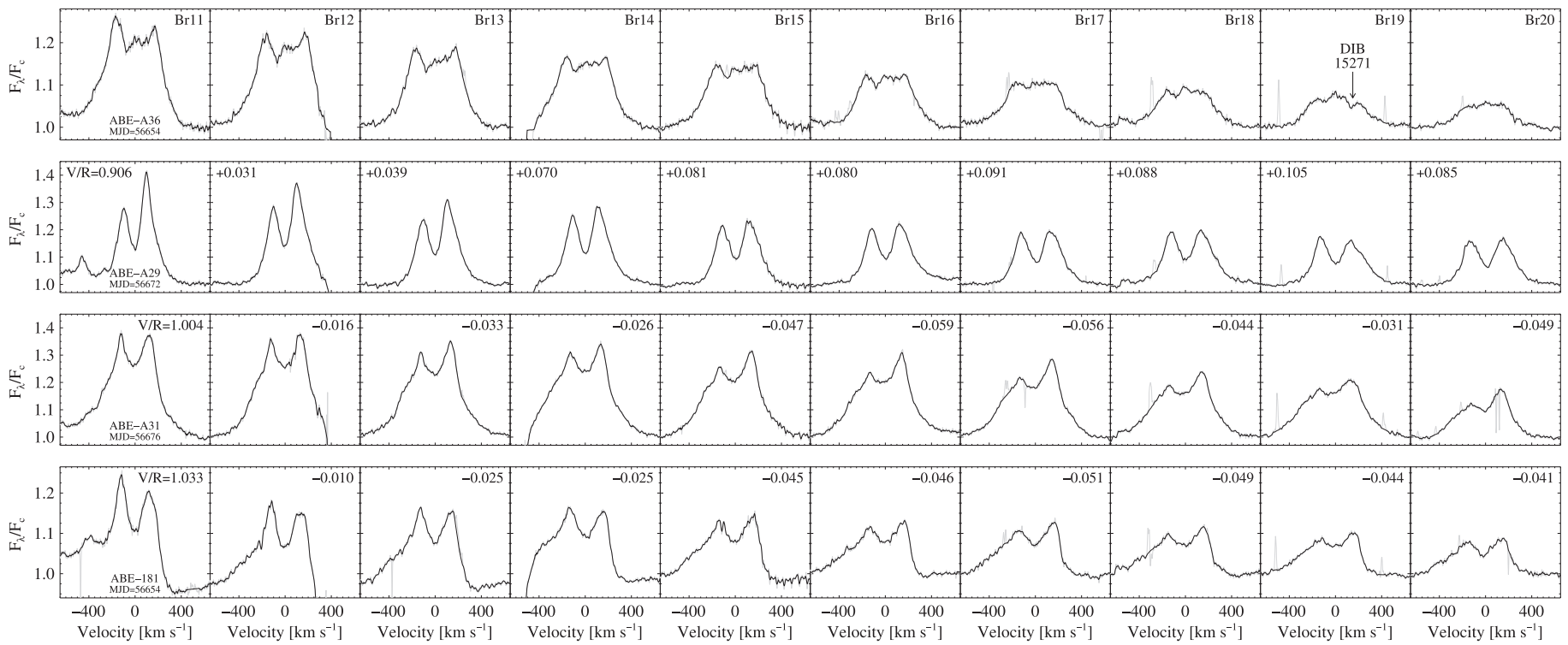

Figure 13. Four examples of variation in V/R phase across the Brackett series lines. The Br11 profile for ABE-A36 has a quasi-triple-peaked morphology which gradually becomes a single-peaked morphology at Br20. For ABE-A29, ABE-A31, and ABE-181, the V/R ratio of Br11 is provided in the leftmost panels and subsequent panels provide the difference between the Br12-Br20 V/R ratios and the Br11 V/R ratio. Gradual changes in V/R orientation are seen among Brackett series lines for these stars: blue text for the differences means increasing V/R ratio from $\mathrm{Br} 11$ to $\mathrm{Br} 20$ (ABE-A29) while red text means decreasing V/R ratio from Br11 to Br20. DIB 15271 absorption is evident on the Br19 line for all four stars.

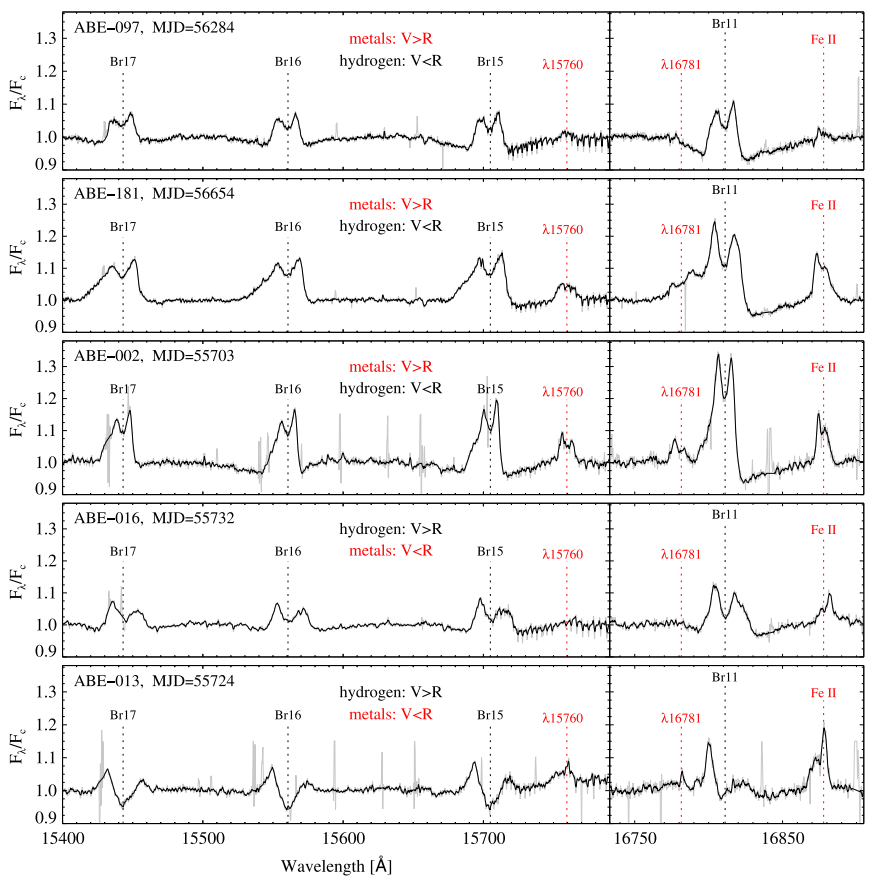

Figure 14. $H_{I}$ vs. metallic $V / R$ orientation mismatches are evident in the APOGEE spectra of ABE-097, ABE-181, ABE-002, ABE-016, and ABE-013. The emission wings for ABE-181 and ABE-002 are also clearly extended in the direction of the weaker emission peak for each line, and the metallic emission profiles for ABE-013 appear to be offset in radial velocity from the Brackett lines.

impacted by DIB 15271 but otherwise appears similar to $\mathrm{Br} 18$. Finally, the $\mathrm{Br} 20$ profile is smooth and rounded with only subtle traces of the blue central absorption and middle "emission peak" (the outer emission peaks are no longer visible).

Štefl et al. (2009) pointed out that although qtp $\mathrm{H} \alpha$ profiles occur at certain times during the V/R cycle of $\zeta$ Tau, optically thin lines likes $\mathrm{O}_{\mathrm{I}} 8446$ and the Brackett series never exhibited any evidence of $q t p$. It is therefore unusual that $q t p$ profiles are observed in the Brackett lines for ABE-A36. We can report no additional examples.

\subsection{H I versus Metallic V/R and RV Phase Lags}

The five examples shown in Figure 14 represent the first known examples of disagreement between the V/R orientations of Brackett series versus metallic lines (Fe II 16878, $\lambda 15760$, and $\lambda 16781)$. ABE-097, ABE-181, and ABE-002 have $\mathrm{V}<\mathrm{R}$ for $\mathrm{H}$ I and $\mathrm{V}>\mathrm{R}$ for metallic lines, while ABE016 and $\mathrm{ABE}-013$ have $\mathrm{V}>\mathrm{R}$ for $\mathrm{H} \mathrm{I}$ and $\mathrm{V}<\mathrm{R}$ for metallic lines. Due to the contaminated Br11 profiles for ABE-013, ABE-002, and ABE-181, where the $\mathrm{V}$ peak height has been increased by underlying $\mathrm{Fe}$ II 16792 emission, the left-hand panels of Figure 14 are extended to encompass not only $\lambda 15760$, but also $\mathrm{Br} 15-\mathrm{Br} 17$ to show the typical $\mathrm{H}_{\mathrm{I}} \mathrm{V} / \mathrm{R}$ orientation for each star.

Although the lack of available stellar absorption lines means that precise stellar RV determination is not possible, the spectrum for ABE-013 in Figure 14 has been corrected to rest frame based on the average positions of the deep absorptions in the $\mathrm{Br} 12-\mathrm{Br} 20$ lines, while the other spectra lack the deep $\mathrm{H} \mathrm{I}$ absorptions and therefore were corrected for Doppler shift based on average emission peak shift for the Brackett lines. The result that emerges for ABE-013 is that the Brackett series absorptions do not coincide in RV not with the central depressions in the metallic emission lines as is normally true (see Figure 8), but instead the Brackett series absorptions coincide in RV with the R emission peaks of the metallic lines. More specifically, the Fe II $16878, \lambda 15760$, and $\lambda 16781$ profiles are shifted in RV with respect to $\mathrm{H}_{\mathrm{I}}$ absorption by $\sim 50 \mathrm{~km} \mathrm{~s}^{-1}$ in the direction of stronger $\mathrm{HI}$ peaks as expected from Okazaki (1991).

ABE-002 and ABE-181 exhibit clear evidence of V/Rrelated RV shifts in the emission profiles, though of a slightly different variety from that of ABE-013. Metallic and $\mathrm{H}_{\mathrm{I}}$ 

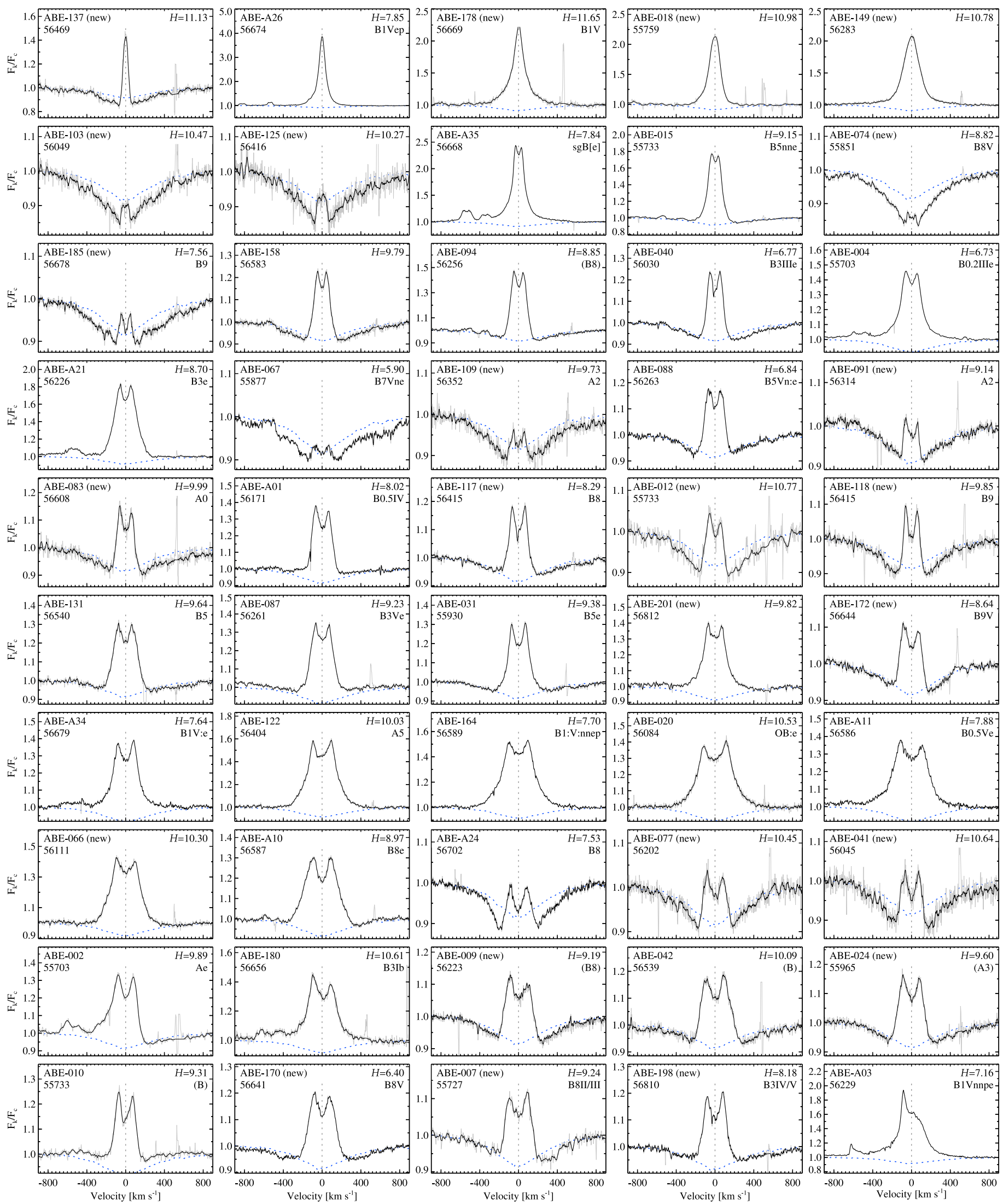

Figure 15. Br11 line profiles sorted approximately according to inclination angle, from pole-on to edge-on. ABE identifiers, observation MJDs, 2MASS $H$ magnitudes, and literature spectral types (where available) are printed in each panel. The average Br11 profile of the quiescent Be stars (ABE-Q01-ABE-Q23) is displayed as a dotted line (blue), and vertical dotted lines (gray) indicate emission peak midpoints or estimated line centers if emission peaks are not present. 

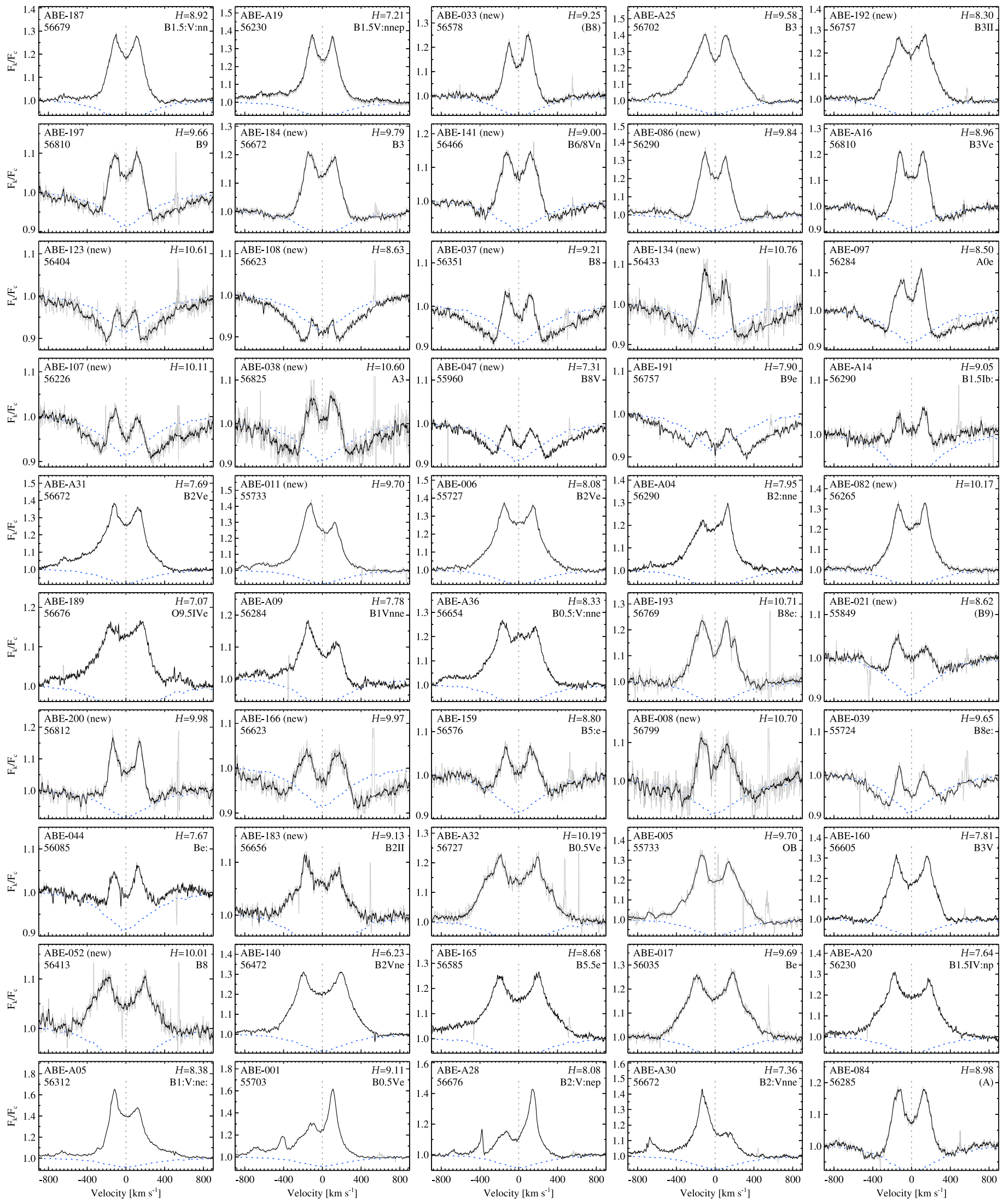

Figure 15. (Continued.) 

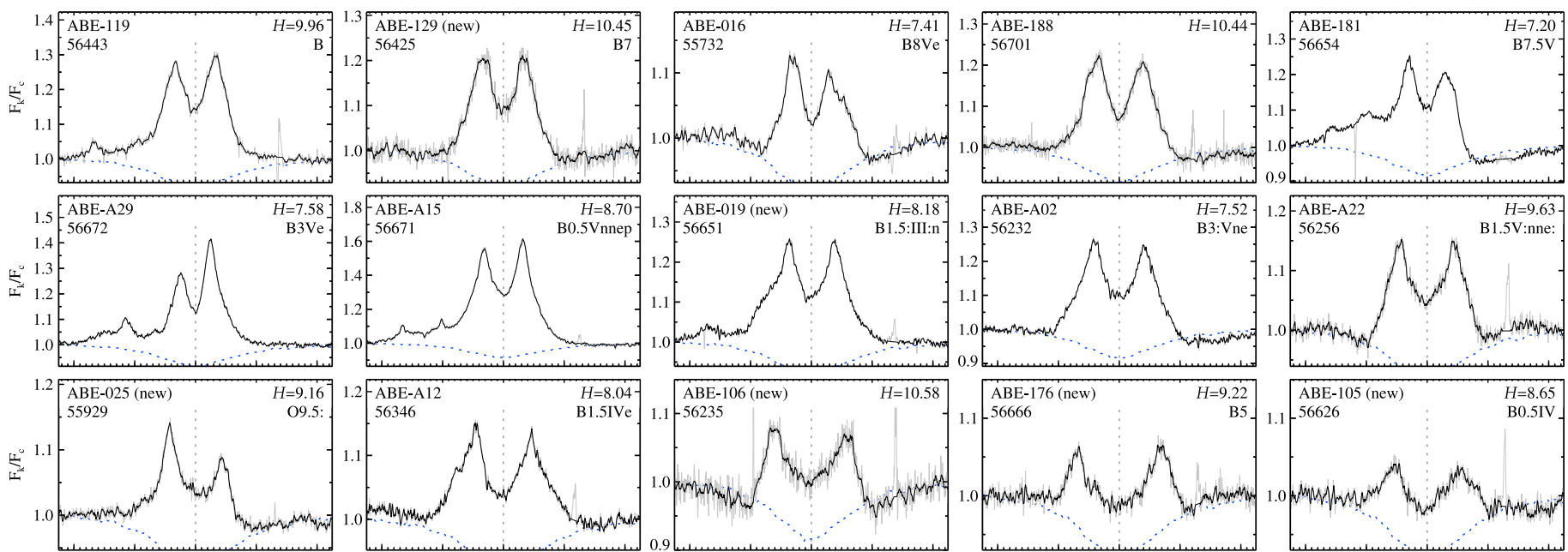

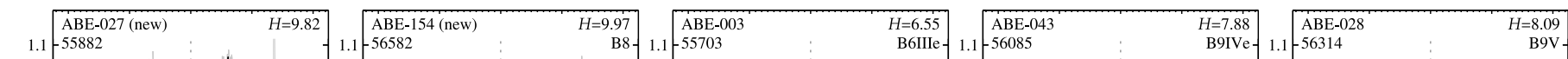

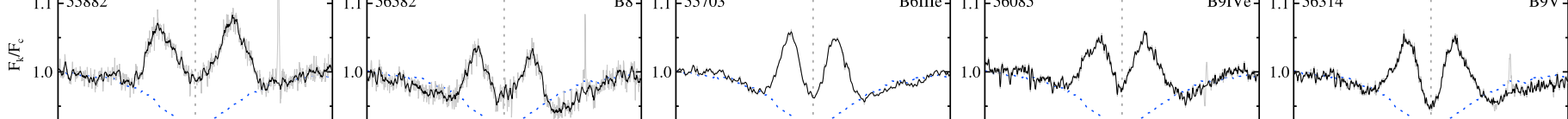

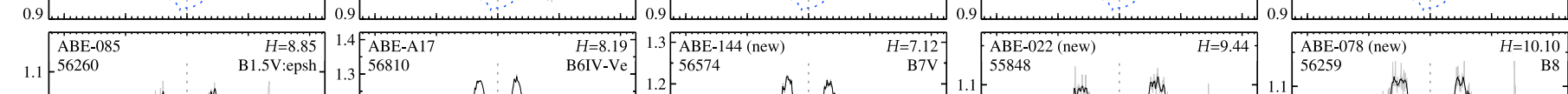
1.1.56260
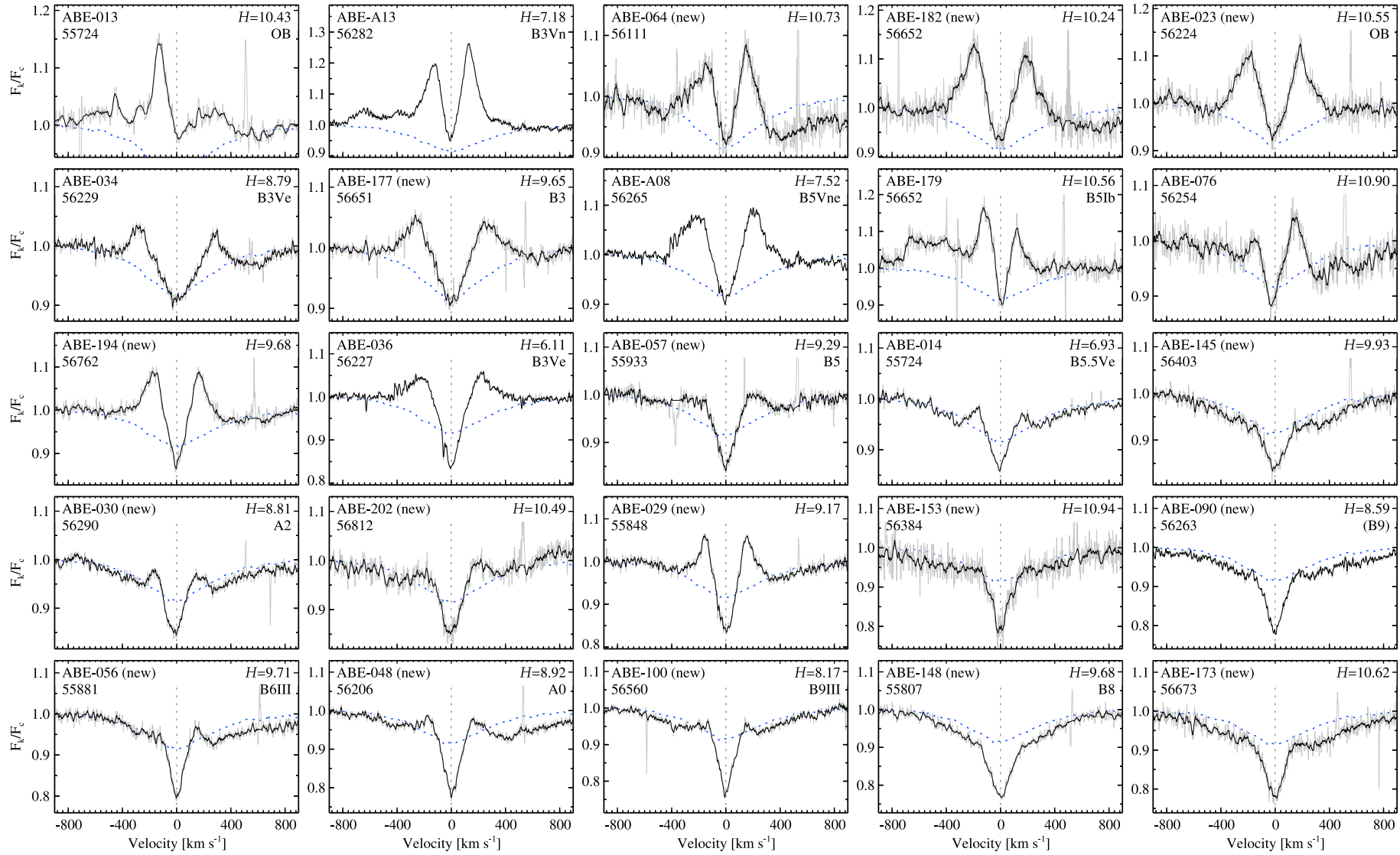

Figure 15. (Continued.) 

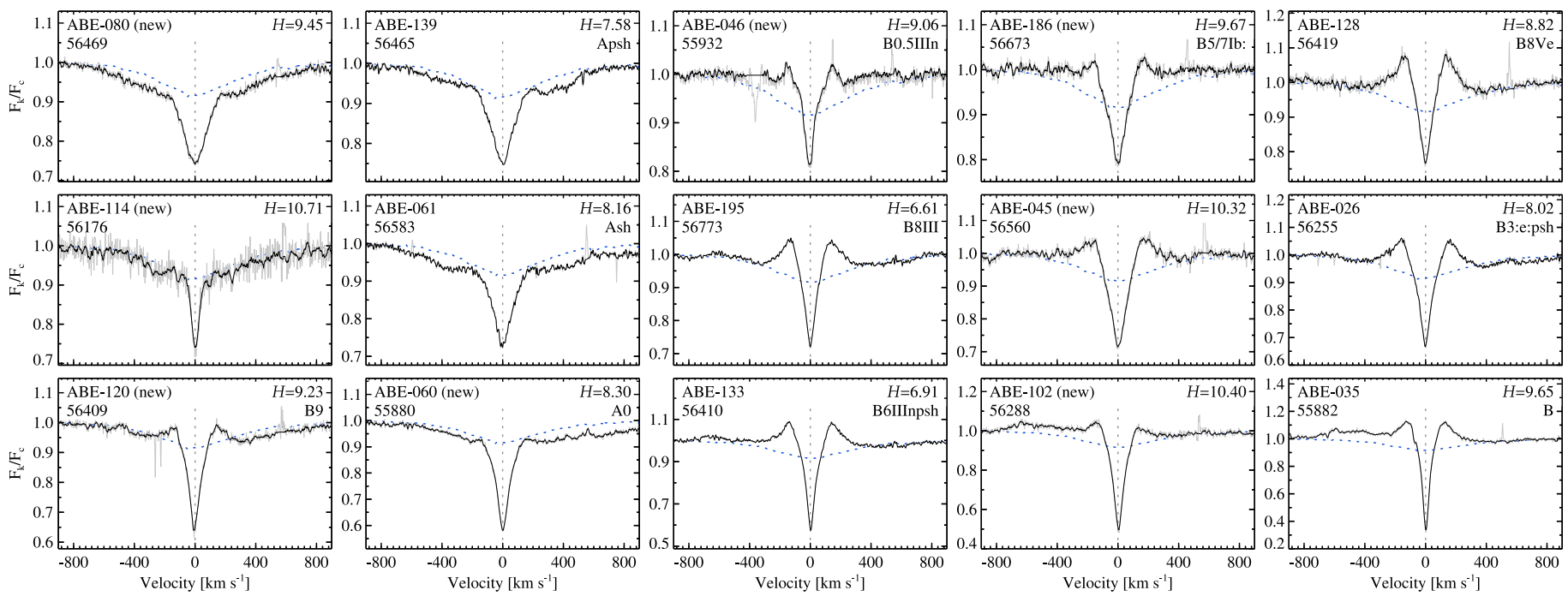

Figure 15. (Continued.)

emission wings for both stars are conspicuously enhanced on the side of the line profiles opposite the stronger emission peak for ABE-002 and ABE-181, with the $\mathrm{H}_{\mathrm{I}}$ wings being enhanced on the blue side and the metallic wings enhanced on the red side. The enhanced blue wings suggest that significantly more emission is being formed in the inner regions of the approaching side of the disk, and the steep declines in intensity, from stronger emission peak to narrower emission base ( $\mathrm{R}$ side of $\mathrm{H}_{\mathrm{I}}$ for $\mathrm{ABE}-002$ and ABE-181), imply cavities in the inner regions of the receding sides of the disks and relatively increased emission coming from the outer regions of the disks. We interpret these line profiles to suggest more tightly wound spiral patterns to the density oscillation in the disks of these stars versus $\zeta$ Tau.

\section{BR11 LINE PROFILES}

Br11 line profiles from the highest-quality-available spectrum of each ABE star are displayed in Figure 15. In Figure 15, the Br11 profiles of $165 \mathrm{ABE}$ stars are qualitatively sorted by profile type, going from single-peaked and narrow double-peaked profiles to deep shell profiles. According to the models of Hummel \& Dachs (1992) and Hummel \& Vrancken (2000), the major line profile shape differences for Be stars are an effect of the inclination angles $(i)$ at which the circumstellar disks are observed. Hanuschik et al. (1996) used high-resolution $\mathrm{H} \alpha$ and optical Fe II profiles to devise a Be sub-classification scheme based on the notion of $i$ dictating to a large extent line profile morphology. Silaj et al. (2010) later showed that line shape in an optically-thick line like $\mathrm{H} \alpha$ is not dictated solely by $i$ and that very different profile shapes may be observed at fixed $i$, but no such investigations of the Brackett series lines have been done.

In sorting the Br11 profiles of the ABE stars according to expected $i$, we relied largely on the models of optically thin lines from Hummel \& Dachs (1992). The most readily classified Br11 profiles correspond to $i \sim 0^{\circ}$ (pole-on), where single-peaked or narrow double-peaked emission is expected, and $i \sim 90^{\circ}$ (edgeon), where deep shell absorption with a sharp core (with or without adjacent emission) is expected. The situation is far more ambiguous for profiles corresponding to intermediate $i$, but a general trend of increasing central depression depth and overall line width with increasing $i$ is apparent. Line profiles that could not be satisfactorily sorted by $i$, due to weakness or ambiguity of the disk features, are shown in Figures 16 and 17. Figure 16 profiles are sorted by Br11 peak separation, and Figure 17 profiles are sorted by $\mathrm{ABE}$ identifier.

\section{CONCLUSIONS}

SDSS-III/APOGEE has serendipitously provided the first high-resolution view of the $H$-band properties of a large number of Be stars, the majority of which are targeted quasirandomly by the survey as telluric calibrators. Although significant progress has been made toward understanding $\mathrm{Be}$ stars over the past few decades via high-resolution optical, interferometric, and spectropolarimetric studies (Rivinius et al. 2013b), any fully explanatory model of the classical Be phenomenon will need to account for the multi-wavelength properties of these stars. Multi-wavelength studies of statistically-significant samples of $\mathrm{Be}$ stars are critical yet have historically been few and far between, though the limited exceptions (Clark \& Steele 2000; Steele \& Clark 2001) have been highly valuable. Due to simultaneous coverage in the $H$ band of numerous $\mathrm{H}_{\mathrm{I}}$ lines that are minimally affected by underlying photospheric absorption in comparison to the Balmer series lines, the $H$-band is particularly promising in terms of utility toward V/R variability and general Be disk studies. Despite the $H$-band covering only a limited number of metallic emission lines, we have shown that the Fe II and Fe IIlike $(\lambda 15760$ and $\lambda 16781)$ lines are highly interesting in the context of $\mathrm{V} / \mathrm{R}$ variability and phase lags between various atomic species.

In the first of a series of papers exploring the $H$-band properties of $\mathrm{Be}$ stars, we have identified the non-hydrogen emission line content of the ABE star spectra, analyzed the kinematic properties of the metallic and $\mathrm{H}_{\mathrm{I}}$ features, and discussed the more exceptional Be stars within the sample as well as those deviating from the typical emission line content. Further investigation of the identities of emission lines at $15760 \AA$ and $16781 \AA$ is needed, but may require updated atomic line lists. Since little is known about most of the ABE stars themselves, including spectral type and rotation speed, optical follow-up study of these stars is also needed in order to develop a better understanding of $H$-band properties as they relate to known stellar parameters. 

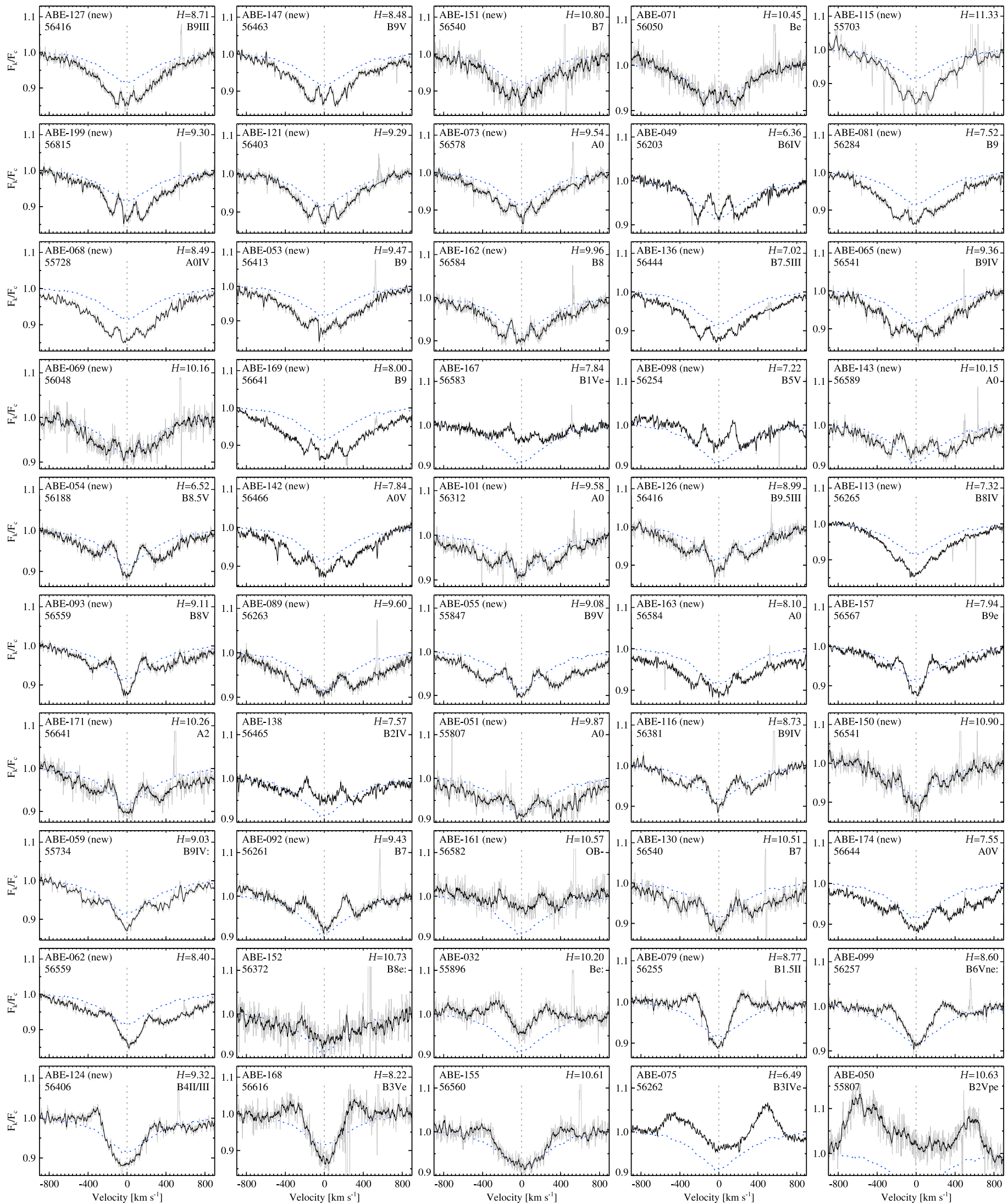

Figure 16. Br11 line profiles for stars with weak or ambiguous emission profile type, as well as for the $\sigma$ Ori E type stars ABE-075 and ABE-050. The panels are sorted by Br11 peak separation. Meanings are otherwise the same as in Figure 15. 

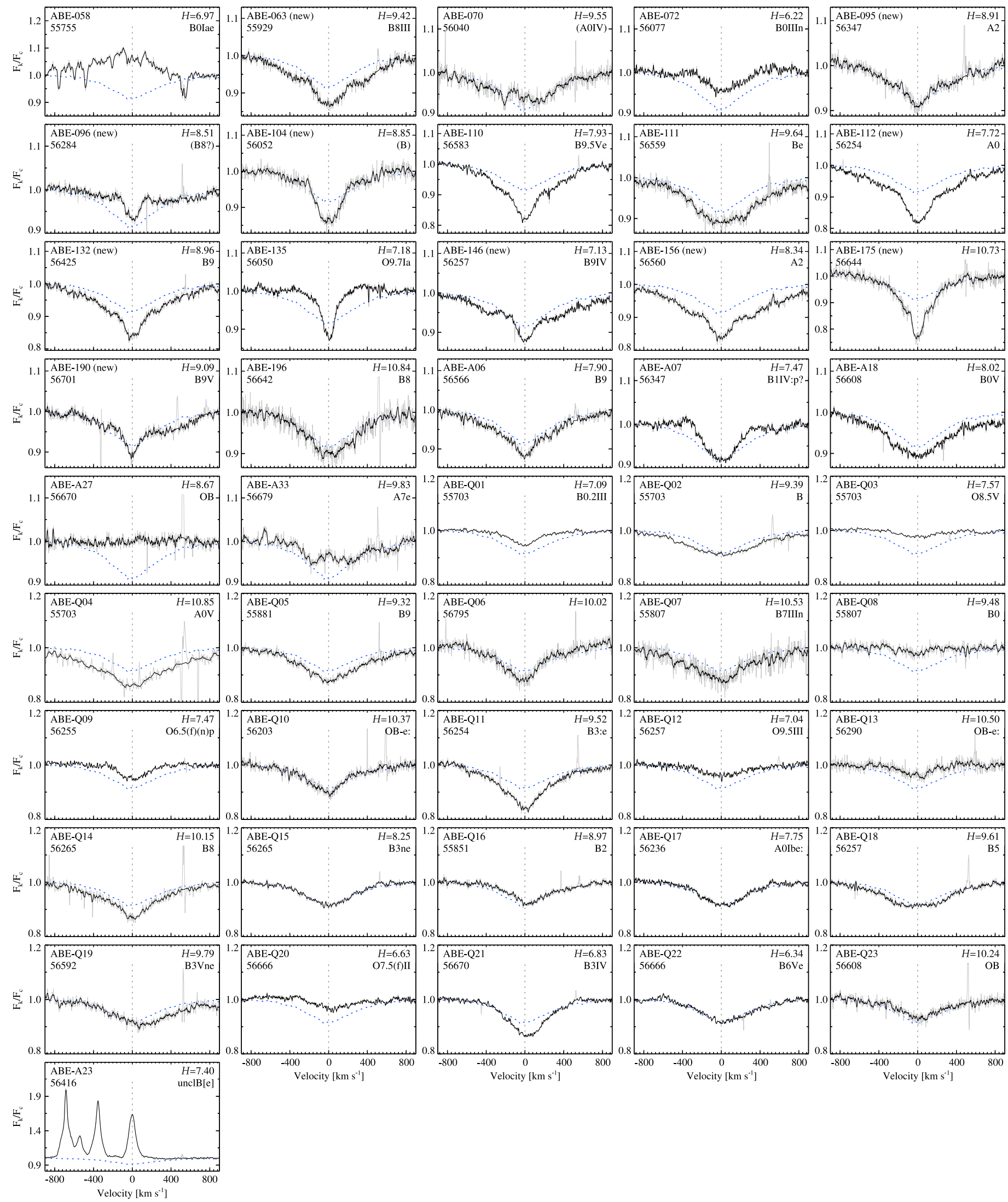

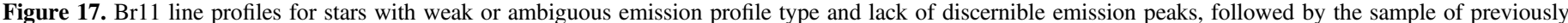

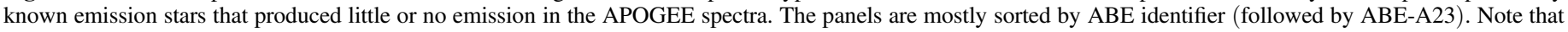

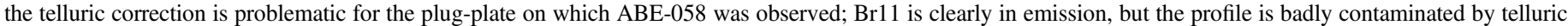
absorption features. Meanings are otherwise the same as in Figure 15. 
Table A1

List of ABE stars

\begin{tabular}{|c|c|c|c|c|c|c|c|c|c|c|c|c|c|}
\hline \multicolumn{4}{|c|}{ Identifiers } & \multicolumn{2}{|c|}{ Magnitudes } & \multicolumn{2}{|c|}{ Spectral type } & \multicolumn{6}{|c|}{ Line detection or $\Delta v_{\mathrm{p}}\left[\mathrm{km} \mathrm{s}^{-1}\right]$} \\
\hline $\mathrm{ABE}$ & 2MASS & HD & Other & $V$ & $H$ & & Ref. & Br11 16811 & $\lambda 15760$ & $\lambda 16781$ & Fe II 16792 & Fe II 16878 & C I 16895 \\
\hline 001 & $20212485+3722482$ & $\ldots$ & VES 203 & 12.09 & 9.108 & $\mathrm{~B} 0.5 \mathrm{Ve}$ & 74 & 208 & 246 & 261 & - & w & - \\
\hline 002 & $20151525+3654562$ & 228576 & AS 394 & 11.41 & 9.888 & $\mathrm{Ae}$ & 29 & 152 & w & 86 & - & 85 & - \\
\hline 003 & $20162816+3703229$ & 192987 & HR 7757 & 6.46 & 6.548 & B6IIIe & 22 & 305 & - & - & - & w? & $\mathrm{w}$ \\
\hline 004 & $20234596+3830033$ & 229221 & MWC 344 & 9.22 & 6.734 & B0.2IIIe & 66 & 115 & 131 & 105 & - & w? & 191 \\
\hline 005 & $20184170+3759106$ & $\ldots$ & Hen 3-1876 & 11.37 & 9.699 & $\mathrm{OB}$ & 6 & 271 & w & w & - & - & - \\
\hline
\end{tabular}

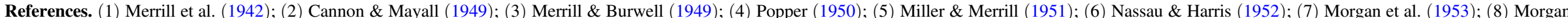

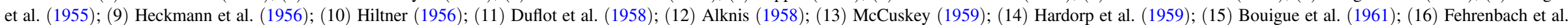

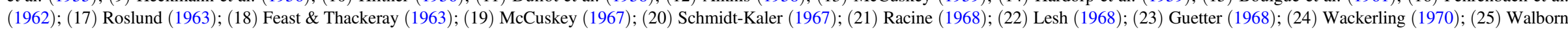

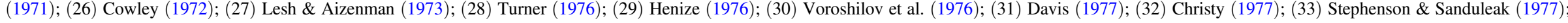

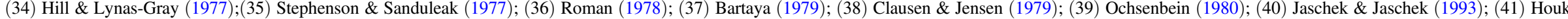

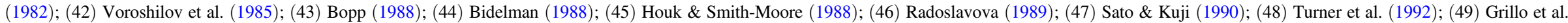

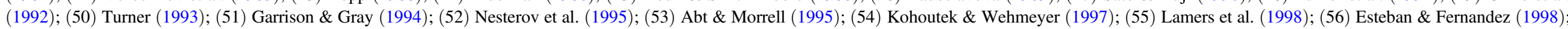

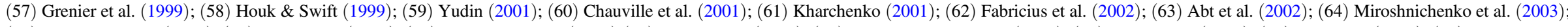

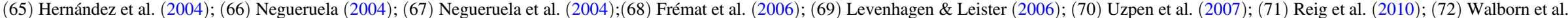
(2010); (73) Sota et al. (2011); (74) Mathew \& Subramaniam (2011); (75) Sebastian et al. (2012); (76) Chargeishvili et al. (2013); (77) Eikenberry et al. (2014).

(This table is available in its entirety in machine-readable and Virtual Observatory (VO) forms.) 


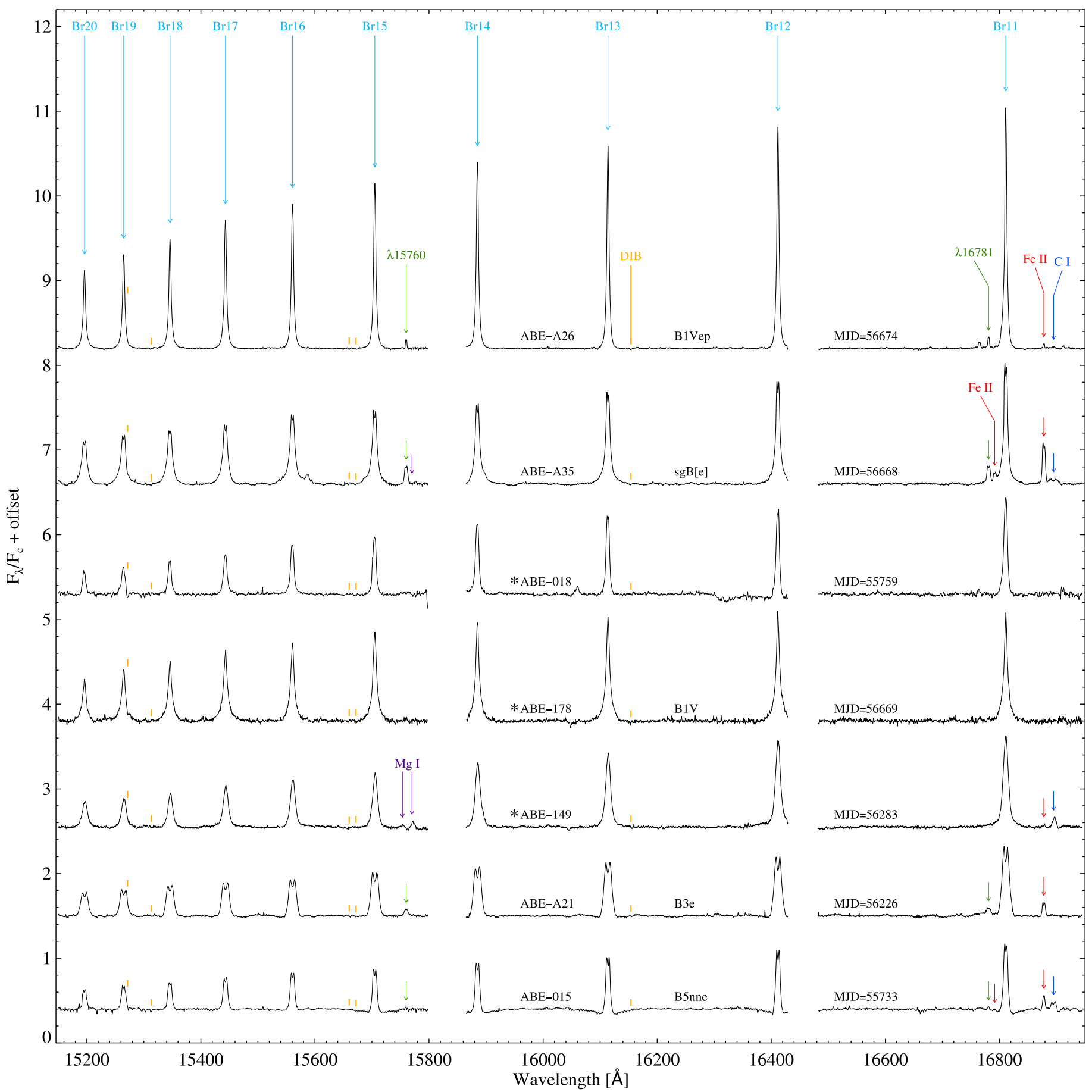

Figure A1. APOGEE spectra for Be stars with strong Brackett series emission. The emission lines for ABE-A35, ABE-A21, and ABE-015 are double-peaked, whereas the other stars have single-peaked emission.

\section{APPENDIX}

Full APOGEE spectra for stars with strong Brackett series features are displayed in Figures A1-A3. Shown above each spectrum are ABE identifiers, observation MJDs, and literature spectral types for each star. Newly identified Be stars are indicated with an asterisk before the ABE identifiers. Small line segments mark the positions of the most prominent DIBs present for some of the stars and arrows mark the positions of the most frequently detected emission lines.

Funding for SDSS-III has been provided by the Alfred P. Sloan Foundation, the Participating Institutions, the National Science Foundation, and the U.S. Department of Energy Office of Science. The SDSS-III web site is http://www.sdss3.org/. SDSSIII is managed by the Astrophysical Research Consortium for the Participating Institutions of the SDSS-III Collaboration including 


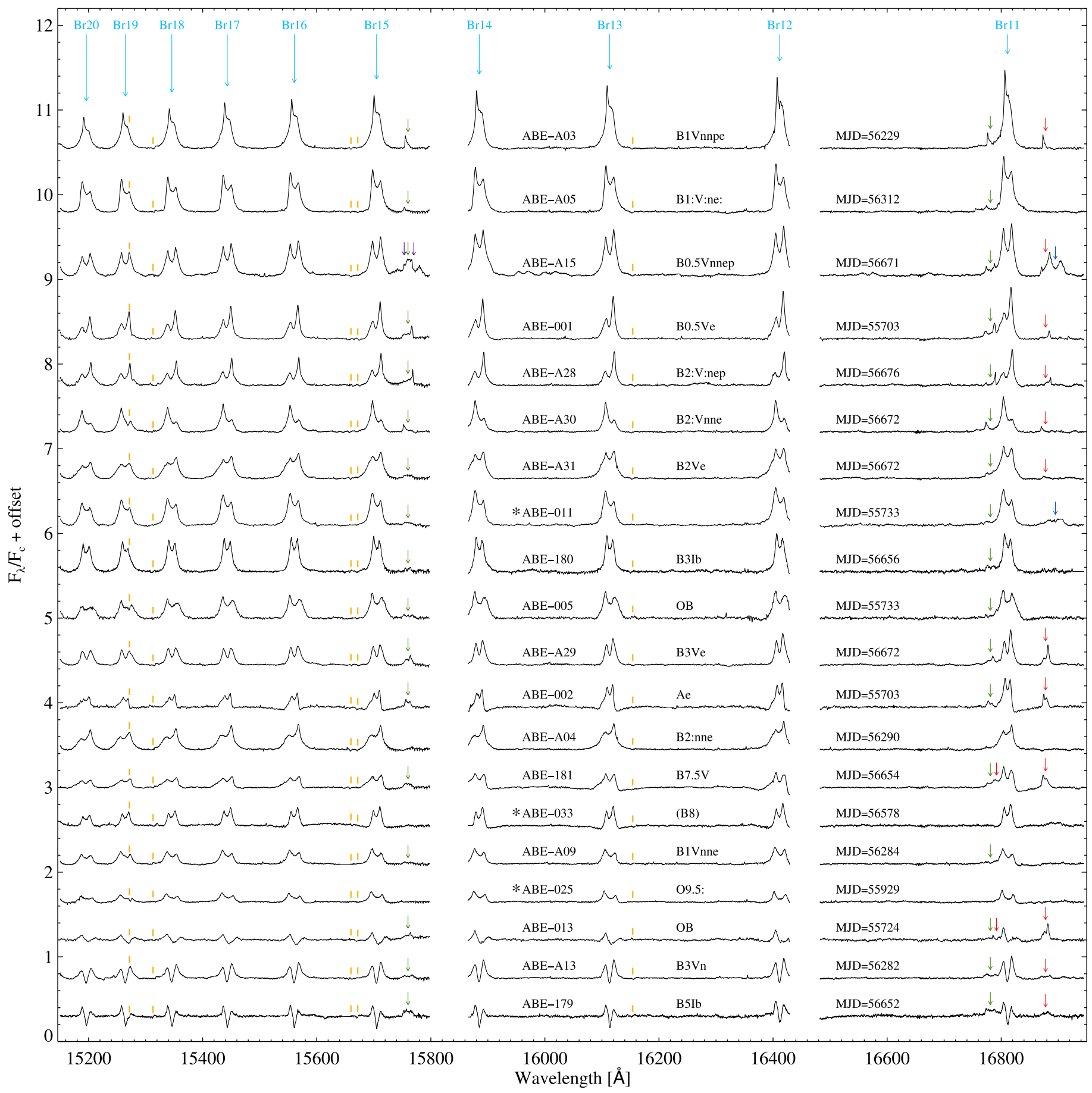

Figure A2. APOGEE spectra for a selection of 20 Be stars with asymmetric Brackett series emission. Note the striking similarity between the spectra of ABE-001, $\mathrm{ABE}-\mathrm{A} 28$, and ABE-A30 (the latter is a V/R reflection of the former two).

the University of Arizona, the Brazilian Participation Group, Brookhaven National Laboratory, Carnegie Mellon University, University of Florida, the French Participation Group, the German Participation Group, Harvard University, the Instituto de Astrofisica de Canarias, the Michigan State/Notre Dame/JINA Participation Group, Johns Hopkins University, Lawrence Berkeley National Laboratory, Max Planck Institute for Astrophysics, Max Planck Institute for Extraterrestrial Physics, New Mexico State University, New York University, Ohio State
University, Pennsylvania State University, University of Portsmouth, Princeton University, the Spanish Participation Group, University of Tokyo, University of Utah, Vanderbilt University, University of Virginia, University of Washington, and Yale University. J. P. W. acknowledges support from NSF-AST 1412110. We thank the anonymous referee and Kevin Covey, both of whom provided feedback that substantially improved the paper. The first author additionally thanks his mother for proofreading drafts of the paper. 


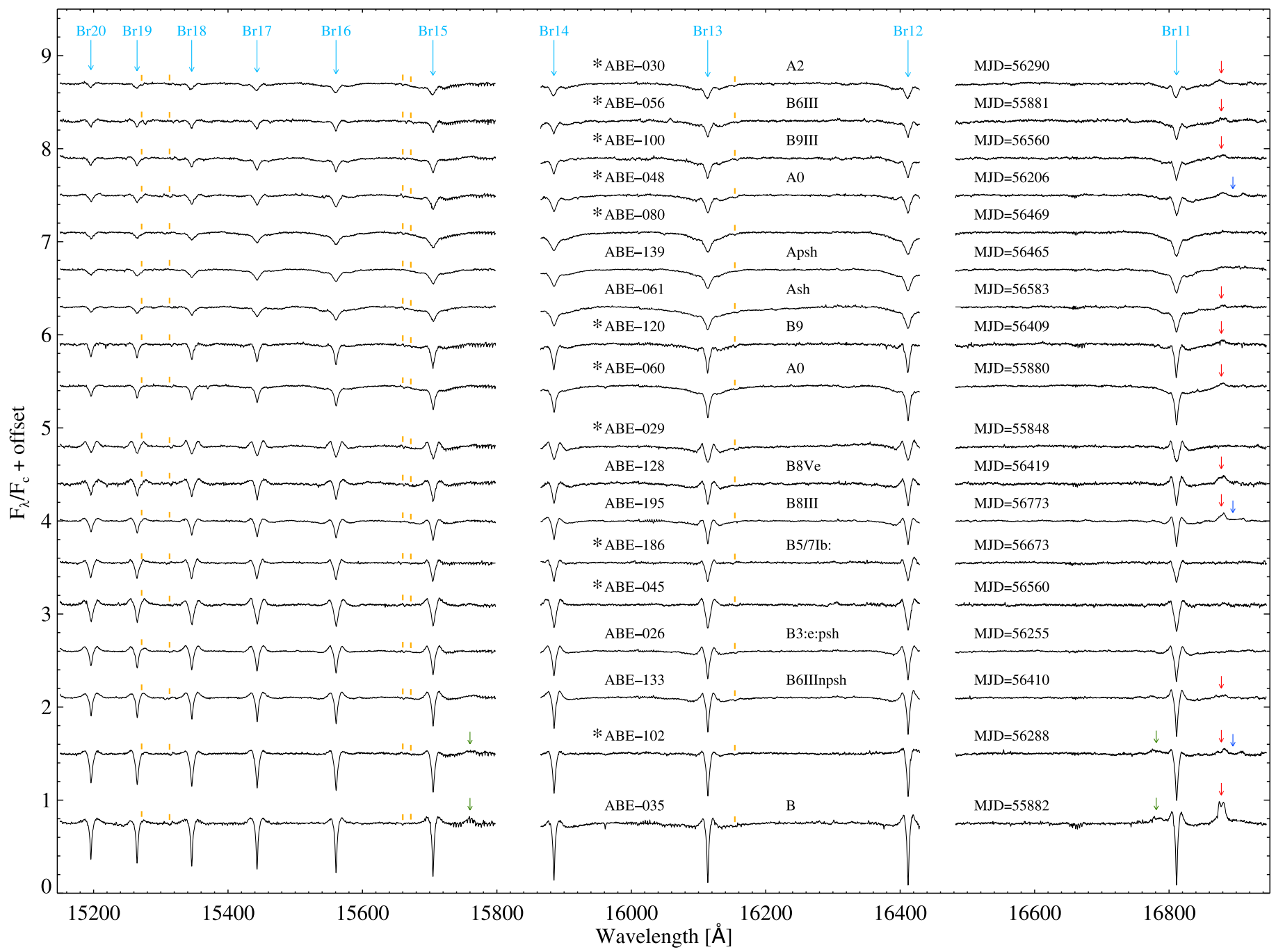

Figure A3. APOGEE spectra for a selection of 18 Be-shell stars. Broad photospheric absorption wings are clearly visible in the Brackett lines for the upper nine stars, while the lower nine stars exhibit mostly smooth continua and shell features with adjacent emission.

\section{REFERENCES}

Abt, H. A., Levato, H., \& Grosso, M. 2002, ApJ, 573, 359

Abt, H. A., \& Morrell, N. I. 1995, ApJS, 99, 135

Alknis, A. 1958, TrRig, 7, 33

Allen, D. A., \& Swings, J. P. 1976, A\&A, 47, 293

Andrillat, A., Jaschek, M., \& Jaschek, C. 1990, A\&AS, 84, 11

Arias, M. L., Zorec, J., Cidale, L., et al. 2006, A\&A, 460, 821

Ashok, N. M., \& Banerjee, D. P. K. 2000, in ASP Conf. Ser. 214, The Be

Phenomenon in Early-Type Stars, ed. M. A. Smith et al. (San Francisco,

CA: ASP), 468

Bartaya, R. A. 1979, AbaOB, 51, 1

Bjorkman, K. S., \& Miroshnichenko, A. S. 2000, BAAS, 32, 1480

Bhavya, B., Mathew, B., \& Subramaniam, A. 2007, BAAS, 35, 383

Bidelman, W. P. 1988, PASP, 100, 1084

Blum, R. D., Ramond, T. M., Conti, P. S., Figer, D. F., \& Sellgren, K. 1997, AJ, 113, 1855

Bopp, B. W. 1988, AJ, 95, 1543

Bouigue, R., Boulon, J., \& Pedoussaut, A. 1961, AnTou, 28, 33

Cannon, A. J., \& Mayall, M. W. 1949, AnHar, 112, 1

Carciofi, A. C., Okazaki, A. T., le Bouquin, J.-B., et al. 2009, A\&A, 504, 915

Carciofi, A. C. 2011, IAUS, 272, 325

Chargeishvili, K. B., Bartaya, R. A., \& Kharadze, E. K. 2013, VizieR Online Data Catalog, 3271, 0

Chauville, J., Zorec, J., Ballereau, D., et al. 2001, A\&A, 378, 861

Christy, J. W. 1977, ApJ, 217, 127
Clark, J. S., \& Steele, I. A. 2000, A\&A, 141, 65

Clausen, J. V., \& Jensen, K. S. 1979, RA, 9, 479

Covey, K. R., Cottaar, M., Foster, J. B., et al. 2014, AASM, 223, 442.10

Cowley, A. 1972, AJ, 77, 750

Dachs, J., Hummel, W., \& Hanuschik, R. W. 1992, A\&AS, 95, 437

Davis, R. J. 1977, ApJ, 213, 105

Duflot, M., Fehrenbach, C., Duflot, A., Rouviere, E., \& Schneider, D. 1958, JO, 41, 43

Eikenberry, S. S., Chojnowski, S. D., Wisniewski, J., et al. 2014, ApJL, 784, L30

Eisenstein, D. J., Weinberg, D. H., Agol, E., et al. 2011, AJ, 142, 72

Esteban, C., \& Fernandez, M. 1998, MNRAS, 298, 185

Fabricius, C., Makarov, V. V., Knude, J., \& Wycoff, G. L. 2002, A\&A, 386, 709

Feast, M. W., \& Thackeray, A. D. 1963, MmRAS, 68, 173

Fehrenbach, C., Rebeirot, E., Petit, M., Peyrin, Y., \& Monvoisin, C. 1962, JO, 45,349

Frémat, Y., Zorec, J., Hubert, A.-M., \& Floquet, M. 2005, A\&A, 440, 305

Frémat, Y., Neiner, C., Hubert, A.-M., et al. 2006, A\&A, 451, 1053

Garrison, R. F., \& Gray, R. O. 1994, AJ, 107, 1556

Geballe, T. R., Najarro, F., Figer, D. F., Schlegelmilch, B. W., \& de La Fuente, D. 2011, Natur, 479, 200

Granada, A., Arias, M. L., \& Cidale, L. S 2010, AJ, 139, 1983

Gray, R. O, \& Corbally, C. J. 2009, Stellar Spectral Classification, ed. Richard O. Gray, \& Christopher J. Corbally (Princeton, NJ: Princeton Univ. Press) 
Greenstein, J. L., \& Wallerstein, G. 1958, ApJ, 127, 237

Grenier, S., Baylac, M.-O., Rolland, L., et al. 1999, A\&AS, 137, 451

Grillo, F., Sciortino, S., Micela, G., Vaiana, G. S., \& Harnden, F. R., Jr. 1992, ApJS, 81, 795

Groh, J. H., Damineli, A., \& Jablonski, F. 2007, A\&A, 465, 993

Guetter, H. H. 1968, PASP, 80, 197

Gunn, J. E., Siegmund, W. A., Mannery, E. J., et al. 2006, AJ, 131, 2332

Halbedel, E. M. 1996, PASP, 108, 833

Hamann, F., Depoy, D. L., Johansson, S., \& Elias, J. 1994, ApJ, 422, 626

Hanuschik, R. W. 1987, A\&A, 173, 299

Hanuschik, R. W. 1988, A\&A, 190, 187

Hanuschik, R. W., Kozok, J. R., \& Kaiser, D. 1988, A\&A, 189, 147

Hanuschik, R. W., Hummel, W., Sutorius, E., Dietle, O., \& Thimm, G. 1996, A\&AS, 116, 309

Hanuschik, R. W. 1996, A\&A, 308, 170

Hardorp, J., Rohlfs, K., Slettebak, A., \& Stock, J. 1959, LS, 0,

Heckmann, O., Dieckvoss, W., \& Kox, H. 1956, AN, 283, 109

Heintz, W. D. 1998, ApJS, 117, 587

Henize, K. G. 1976, ApJS, 30, 491

Hernández, J., Calvet, N., Briceño, C., Hartmann, L., \& Berlind, P. 2004, AJ, 127,1682

Hill, P. W., \& Lynas-Gray, A. E. 1977, MNRAS, 180, 691

Hiltner, W. A. 1947, ApJ, 105, 212

Hiltner, W. A. 1956, ApJS, 2, 389

Hony, S., Waters, L. B. F. M., Zaal, P. A., et al. 2000, A\&A, 355, 187

Horne, K., \& Marsh, T. R. 1986, MNRAS, 218, 761

Houk, N., \& Smith-Moore, M. 1988, Michigan Catalogue of Two-dimensional Spectral Types for the HD Stars. (Ann Arbor, MI: Univ. of Michigan)

Houk, N., \& Swift, C. 1999, in Michigan Catalogue of Two-dimensional Spectral Types for the HD Stars. (Ann Arbor, MI: Univ. of Michigan)

Houk, N. 1982, Michigan Catalogue of Two-dimensional Spectral Types for the HD stars. (Ann Arbor, MI: Univ. of Michigan)

Huang, S.-S. 1972, ApJ, 171, 549

Huang, W., Gies, D. R., \& McSwain, M. V. 2010, ApJ, 722, 605

Huang, W., \& Gies, D. R. 2006, ApJ, 648, 580

Hummel, W., \& Dachs, J. 1992, A\&A, 262, L17

Hummel, W., \& Vrancken, M. 2000, A\&A, 359, 1075

Jaschek, C., \& Jaschek, M. 1993, A\&AS, 97, 807

Kendall, T. R., de Wit, W. J., \& Yun, J. L. 2003, A\&A, 408, 313

Kharchenko, N. V. 2001, KFNT, 17, 409

Kohoutek, L., \& Wehmeyer, R. 1997, AAHam 11

Kramida, A., Ralchenko, Yu., \& Reader, J. 2013, NIST ASD Team, NIST Atomic Spectra Database, (version 5.1), [Online]. Available: http://physics. nist.gov/asd [Thursday, 26-Dec-2013 13:32:45 EST]. National Institute of Standards and Technology, Gaithersburg, MD.

Kraus, S., Calvet, N., Hartmann, L., et al. 2012, ApJ, 752, 11

Lamers, H. J. G. L. M., Zickgraf, F.-J., de Winter, D., Houziaux, L., \& Zorec, J. 1998, A\&A, 340, 117

Lee, U., Osaki, Y., \& Saio, H. 1991, MNRAS, 250, 432

Lesh, J. R., \& Aizenman, M. L. 1973, A\&A, 22, 229

Lesh, J. R. 1968, ApJS, 17, 371

Levenhagen, R. S., \& Leister, N. V. 2006, MNRAS, 371, 252

Majewski, S. R. 2012, AASM, 219, 205.06

Mathew, B., \& Subramaniam, A. 2011, BASI, 39, 517

Mathew, B., Banerjee, D. P. K., Naik, S., \& Ashok, N. M. 2012, MNRAS, 423, 2486

McCuskey, S. W. 1959, ApJS, 4, 1

McCuskey, S. W. 1967, AJ, 72, 1199

McSwain, M. V., Huang, W., \& Gies, D. R. 2009, ApJ, 700, 1216

Meilland, A., Stee, P., Vannier, M., et al. 2007, A\&A, 464, 59

Mennickent, R. E., Sabogal, B., Granada, A., \& Cidale, L. 2009, PASP, 121,125

Merrill, P. W., \& Burwell, C. G. 1949, ApJ, 110, 387

Merrill, P. W., Burwell, C. G., \& Miller, W. C. 1942, ApJ, 96, 15

Meyer, M. R., Edwards, S., Hinkle, K. H., \& Strom, S. E. 1998, ApJ, 508, 397

Miller, W. C., \& Merrill, P. W. 1951, ApJ, 113, 624

Miroshnichenko, A. S., Kusakin, A. V., Bjorkman, K. S., et al. 2003, A\&A, 412, 219

Morgan, W. W., Whitford, A. E., \& Code, A. D. 1953, ApJ, 118, 318

Morgan, W. W., Code, A. D., \& Whitford, A. E. 1955, ApJS, 2, 41

Murdoch, K. A., Drew, J. E., \& Anderson, L. S. 1994, A\&A, 284, 27

Nassau, J. J., \& Harris, D., III 1952, ApJ, 115, 459

Negueruela, I. 2004, AN, 325, 380

Negueruela, I., Steele, I. A., \& Bernabeu, G. 2004, AN, 325, 749
Neiner, C., de Batz, B., Cochard, F., et al. 2011, AJ, 142, 149

Nesterov, V. V., Kuzmin, A. V., Ashimbaeva, N. T., et al. 1995, A\&AS, 110,367

Ochsenbein, F. 1980, BICDS, 19, 74

Okazaki, A. T. 1991, PASJ, 43, 75

Oksala, M. E., Kraus, M., Cidale, L. S., Muratore, M. F., \& Borges Fernandes, M. 2013, A\&A, 558, A17

Polidan, R. S., \& Peters, G. J. 1976, in IAU Symp. 70, Be and Shell Stars, ed. A. Slettebak (Boston, MA: Reidel), 59

Popper, D. M. 1950, ApJ, 111, 495

Porter, J. M., \& Rivinius, T. 2003, PASP, 115, 1153

Racine, R. 1968, AJ, 73, 233

Radoslavova, T. 1989, AN, 310, 223

Reig, P., Zezas, A., \& Gkouvelis, L. 2010, A\&A, 522, A107

Renson, P., Gerbaldi, M., \& Catalano, F. A. 1991, A\&AS, 89, 429

Rivinius, T., Štefl, S., \& Baade, D. 2006, A\&A, 459, 137

Rivinius, T. 2013, Stellar Pulsations: Impact of New Instrumentation and New Insights, 31, 253

Rivinius, T., Carciofi, A. C., \& Martayan, C. 2013, A\&ARv, 21, 69

Rivinius, T., Baade, D., Townsend, R. H. D., Carciofi, A. C., \& Štefl, S. 2013, A\&A, 559, L4

Roman, N. G. 1978, AJ, 83, 172

Roslund, C. 1963, ArA, 3, 97

Rudy, R. J., Erwin, P., Rossano, G. S., \& Puetter, R. C. 1992, ApJ, 398, 278

Sato, K., \& Kuji, S. 1990, A\&AS, 85, 1069

Schmidt-Kaler, T. 1967, PASP, 79, 181

Sebastian, D., Guenther, E. W., Schaffenroth, V., et al. 2012, A\&A, 541, A34

Silaj, J., Jones, C. E., Tycner, C., Sigut, T. A. A., \& Smith, A. D. 2010, ApJS, 187,228

Skiff, B. A. 2013, VizieR Online Data Catalog, 1, 2023

Skrutskie, M. F., Cutri, R. M., Stiening, R., et al. 2006, AJ, 131, 1163

Slettebak, A., Collins, G. W. II, \& Truax, R. 1992, ApJS, 81, 335

Smak, J. 1969, ACA, 19, 155

Smak, J. 1969, ACA, 31, 395

Smee, S. A., Gunn, J. E., Uomoto, A., et al. 2013, AJ, 146, 32

Smith, N. 2001, Eta Carinae and Other Mysterious Stars: The Hidden Opportunities of Emission Spectroscopy, 242, 81

Smith, N., \& Davidson, K. 2001, ApJL, 551, 401

Sota, A., Maiz Appelániz, J., Walborn, N. R., Alfaro, E. J., Barb’a, R. H., Morrell, N. I., Gamen, R. C., \& Arias, J. L. 2011, ApJS, 193, 24

Steele, I. A., Negueruela, I., \& Clark, J. S. 1999, A\&AS, 137, 147

Steele, I. A., \& Clark, J. S. 2001, A\&A, 371, 643

Štefl, S., Rivinius, T., Carciofi, A. C., et al. 2009, A\&A, 504, 929

Stephenson, C. B., \& Sanduleak, N. 1977, PW\&SO, 2, 71

Stephenson, C. B., \& Sanduleak, N. 1977, ApJS, 33, 459

Strom, S. E., Wolff, S. C., \& Dror, D. H. A. 2005, AJ, 129, 809

Struve, O. 1931, ApJ, 74, 225

Townsend, R. H. D., \& Owocki, S. P. 2005, MNRAS, 357, 251

Townsend, R. H. D., Owocki, S. P., \& Groote, D. 2005, ApJL, 630, L81

Turner, D. G., Forbes, D., \& Pedreros, M. 1992, AJ, 104, 1132

Turner, D. G. 1976, ApJ, 210, 65

Turner, D. G. 1993, A\&AS, 97, 755

Tuthill, P. G., \& Lloyd, J. P. 2007, Sci, 316, 247

Uesugi, A., \& Fukuda, I. 1970, Catalogue of Rotational Velocities of the Stars, (Kyoto: Univ. of Kyoto)

Uesugi, A., \& Fukuda, I. 1982, Catalogue of Stellar Rotational Velocities, (rev. ed.; Kyoto: Univ. of Kyoto)

Uzpen, B., Kobulnicky, H. A., Monson, A. J., et al. 2007, ApJ, 658, 1264

Voroshilov, V. I., Guseva, N. G., Kalandadze, N. B., et al. 1976, KiIND

Voroshilov, V. I., Guseva, N. G., Kalandadze, N. B., et al. 1985, KiIND 140 In Russian

Wackerling, L. R. 1970, MmRAS, 73, 153

Walborn, N. R., Howarth, I. D., Evans, C. J., et al. 2010, AJ, 139, 1283

Walborn, N. R. 1971, ApJS, 23, 257

Wheelwright, H. E., Bjorkman, J. E., Oudmaijer, R. D., et al. 2012, MNRAS, 423, L11

Wilson, J. C., Hearty, F., Skrutskie, M. F., et al. 2010, Proc. SPIE, 7735, $77351 \mathrm{C}$

Wisniewski, J. P., Kowalski, A. F., Bjorkman, K. S., Bjorkman, J. E., \& Carciofi, A. C. 2007, ApJ, 656, 21

Wisniewski, J. P., Draper, Z. H., Bjorkman, K. S., et al. 2010, ApJ, 709, 1306 Yudin, R. V. 2001, A\&A, 368, 912

Zasowski, G., Johnson, J. A., Frinchaboy, P. M., et al. 2013, AJ, 146, 81

Zasowski, G., Ménard, B., Bizyaev, D., et al. 2014, arXiv:14061195 